\title{
A Tutorial on Interference Exploitation via Symbol-Level Precoding: Overview, State-of-the-Art and Future Directions
}

\author{
Ang Li, Member, IEEE, Danilo Spano, Member, IEEE, Jevgenij Krivochiza, Student Member, IEEE, \\ Stavros Domouchtsidis, Student Member, IEEE, Christos G. Tsinos, Member, IEEE, Christos Masouros, Senior \\ Member, IEEE, Symeon Chatzinotas, Senior Member, IEEE, Yonghui Li, Fellow, IEEE, \\ Branka Vucetic, Life Fellow, IEEE, and Björn Ottersten, Fellow, IEEE
}

\begin{abstract}
Interference is traditionally viewed as a performance limiting factor in wireless communication systems, which is to be minimized or mitigated. Nevertheless, a recent line of work has shown that by manipulating the interfering signals such that they add up constructively at the receiver side, known interference can be made beneficial and further improve the system performance in a variety of wireless scenarios, achieved by symbol-level precoding (SLP). This paper aims to provide a tutorial on interference exploitation techniques from the perspective of precoding design in a multi-antenna wireless communication system, by beginning with the classification of constructive interference (CI) and destructive interference (DI). The definition for $\mathrm{CI}$ is presented and the corresponding mathematical characterization is formulated for popular modulation types, based on which optimization-based precoding techniques are discussed. In addition, the extension of CI precoding to other application scenarios as well as for hardware efficiency is also described. Proof-of-concept testbeds are demonstrated for the potential practical implementation of CI precoding, and finally a list of open problems and practical challenges are presented to inspire and motivate further research directions in this area.
\end{abstract}

Index Terms-MIMO, constructive interference, symbol-level precoding, optimization, application, faster-than-Nyquist, hardware efficiency, proof-of-concept testbed.

\section{INTRODUCTION}

Manuscript received July 03, 2019; revised October 21, 2019; January 15, 2020 and March 04, 2020; accepted March 09, 2020. The associate editor coordinating the review of this paper and approving it for publication was Prof. Fabrizio Granelli. (Corresponding author: Ang Li.)

A. Li is with the Faculty of Electronic and Information Engineering, Xi' an Jiaotong University, Xi'an, China. (e-mail: ang.li.2020@xjtu.edu.cn).

D. Spano, J. Krivochiza, S. Domouchtsidis, C. G. Tsinos, S. Chatzinotas and B. Ottersten are with the Interdisciplinary Centre for Security, Reliability, and Trust, University of Luxembourg, 1855 Luxembourg, Luxembourg. (e-mail: \{danilo.spano, jevgenij.krivochiza, stavros.domouchtsidis, christos.tsinos, symeon.chatzinotas, bjorn.ottersten\}@uni.lu)

C. Masouros is with the Department of Electronic and Electrical Engineering, University College London, Torrington Place, London, WC1E 7JE, UK. (email: c.masouros@ucl.ac.uk)

Y. Li, and B. Vucetic are with the School of Electrical and Information Engineering, University of Sydney, NSW 2006, Australia. (email: \{yonghui.li, branka.vucetic\}@sydney.edu.au)

This work was supported in part by the Engineering and Physical Sciences Research Council (EPSRC) Project under Grant EP/R007934/1, in part by the FNR, Luxembourg under the projects INTER CI-PHY and CORE ECLECTIC, in part by the Australian Research Council (ARC) under Grant DP150104019 and Grant DP190101988, in part by the ARC Laureate Fellowship under Grant FL160100032, and in part by the Science and Technology Program of Shaanxi Province under Grant No. 2019KW-007.
$\mathbf{P}$ RECODING is able to support data transmissions to multiple receivers simultaneously in multi-antenna wireless communication systems, which has attracted significant interest in their development towards 5G [1]. The term 'precoding' usually refers to the transmit signal design that directs the desired data symbols to the intended users while limiting the inter-user interference, by exploiting the channel state information (CSI) and potentially the information of the data symbols. In the literature, the dirty paper coding (DPC) technique is known to be capable of achieving the channel capacity theoretically [2]. Despite its optimality, DPC is difficult to implement in practical wireless communication systems, due to (i) the impractical assumption of an infinite source alphabet and (ii) the prohibitive computational complexity incurred by sequential encoding. Therefore, linear precoding methods, where the precoded signals are linear combinations of the information symbols, have become appealing and attracted more research attention because of their low complexity [3][5]. In the literature, while the maximum ratio transmission (MRT) precoding offers the lowest computational cost [3], it does not fully eliminate the multi-user interference, which leads to an error floor at medium-to-high signal-to-noise ratio (SNR) regions. Zero-forcing (ZF) precoding is able to improve the performance of MRT precoding by fully eliminating the multi-user interference via inverting the channel [4], whose performance can be further improved via the regularized $\mathrm{ZF}$ (RZF) precoding by including a regularization factor in the matrix inversion, which alleviates the noise amplification effect that ZF precoding suffers [5].

In addition to these closed-form precoders, linear precoding methods based on optimization have received increasing research attention recently because of their flexibility to achieve various performance targets, where the most popular two design targets are power minimization (PM) and signalto-interference-plus-noise ratio (SINR) balancing (SB) [6][9]. For unicast applications where the base station (BS) transmits individual information to each receiver, PM aims to minimize the total transmit power at the BS subject to a common minimum SINR target for all the receivers [6] or an individual SINR target for each user [7], while SB targets at maximizing the minimum SINR for each receiver while satisfying a total transmit power requirement [8] or a per-antenna power constraint [9] at the BS. Given the 


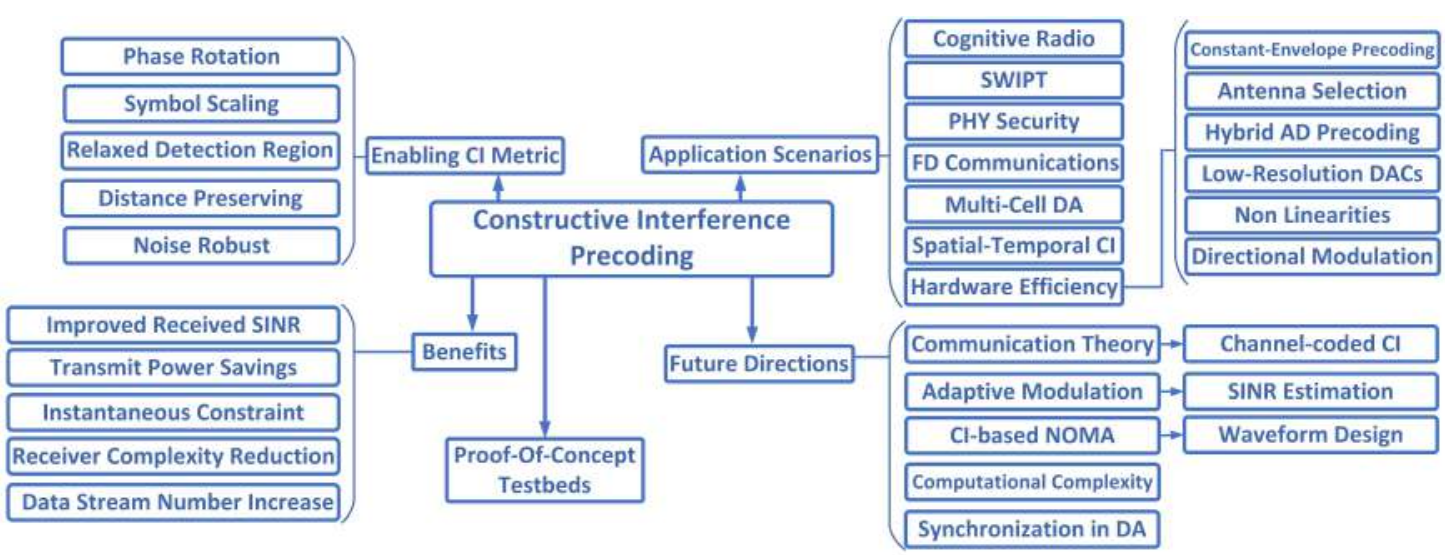

Fig. 1: Various aspects of interference exploitation via symbol-level precoding

capability of adaptation to various wireless communication scenarios, optimization-based precoding schemes have been extended to a variety of research areas such as cognitive radio (CR) [10], simultaneous wireless information and power transfer (SWIPT) [11], [12], physical-layer (PHY) security [13]-[16], full-duplex (FD) communications [17]-[19], radar and communication coexistence [20], [21], etc., which will be overviewed in the corresponding chapters in the following.

For both closed-form linear precoding methods [3]-[5] and optimization-based schemes [6]-[9] described above, it is observed that only the information of the channel is exploited for the precoding design, and these precoding methods all treat interference as a detrimental effect. Nevertheless, it has already been observed in non-linear precoding methods such as Tomlinson-Harashima precoding (THP) [22]-[24] and vector perturbation (VP) precoding [25]-[27] that both the CSI and the data symbols have been included in the symbol-bysymbol precoding design, i.e., the information of the data symbols is also exploited. However, the problem for nonlinear precoding schemes is that they are still difficult to be implemented in practical wireless communication systems, due to the complicated encoding and decoding process that leads to unfavorable computational costs. Therefore, it is natural to ask: Is it possible for linear precoding methods to potentially exploit the information of the data symbols as well, or more specifically exploit the interference based on the knowledge of the data symbols to further improve the performance?

To answer the above question, this paper provides a tutorial on a recently proposed concept termed 'constructive interference' (CI) and the corresponding CI precoding techniques, as well as their applications to a number of current and future wireless communication scenarios, as illustrated in Fig. 1. Compared with a previous survey paper [28] on symbol-level precoding (SLP) which includes 1) the comparison between traditional block-level precoding and emerging SLP regarding their application in both unicast and multicast scenarios, 2) directional modulation based on SLP, 3) the symbol-level PM problem based on CI for PSK modulations, and 4) the transmit architecture for SLP techniques, the focus of this tutorial paper is on 1) the illustration, definition, characterization and classification of CI for different modulation types, 2) the exploitation of the CI effect in a variety of wireless communication scenarios including VP, PM, SB, CR, SWIPT, PHY security, etc., and 3) the proof-of-concept testbeds for CI.

We begin with a brief review on precoding, followed by the introduction of $\mathrm{CI}$, its potential benefits and current limitations in Section I. Section II then introduces the classification and mathematical characterization of CI for various modulation types, based on which Section III formulates the optimization problems for CI exploitation, whose solution can be obtained via convex optimization tools. Section IV describes the applications of CI exploitation techniques in traditional small-scale multiple-input multiple-output (MIMO) systems, and Section $\mathrm{V}$ extends the application to large-scale antenna systems for hardware efficiency. Section VI describe the proof-of-concept testbed for practical implementation of CI exploitation via SLP, developed by University College London and University of Luxembourg, respectively. Section VII discusses some open problems and challenges to be explored, followed by Section VIII that concludes the paper. For clarity, we first summarize the notations that are employed in the subsequent sections in Table I.

\section{A. Preliminaries on Precoding}

Before we introduce $\mathrm{CI}$, in this section we firstly review how precoding works in the downlink transmission of a multi-antenna system as preliminaries, where the traditional $\mathrm{ZF}$ precoding scheme is also introduced as a simple and illustrative example.

For notational convenience, we consider a generic multiuser multiple-input single-output (MU-MISO) system in the downlink, where the BS equipped with $N_{\mathrm{T}}$ transmit antennas communicates with a total number of $K$ single-antenna users in the same time-frequency resource. Since users are usually separate and do not cooperate in the downlink transmission, in order to manage the potential multi-user interference, the BS needs to perform some signal processing techniques on the data symbols prior to transmission based on the CSI, and this is where the term 'precoding' comes from. Mathematically, 
the precoded signal vector $\mathrm{x} \in \mathcal{C}^{N_{\mathrm{T}} \times 1}$ to be transmitted at the antenna ports can be expressed as [6]

$$
\mathbf{x}=\sum_{k=1}^{K} \mathbf{w}_{k} s_{k}=\mathbf{W} \mathbf{s},
$$

where $\mathbf{w}_{k} \in \mathcal{C}^{N_{\mathrm{T}} \times 1}$ is the precoding vector for user $k$ 's data symbol $s_{k}$, which is drawn from a specific modulation constellation. $\mathbf{W}=\left[\mathbf{w}_{1}, \mathbf{w}_{2}, \cdots, \mathbf{w}_{K}\right] \in \mathcal{C}^{N_{\mathrm{T}} \times K}$ is the concatenated precoding matrix and $\mathbf{s}=\left[s_{1}, s_{2}, \cdots, s_{K}\right]^{\mathrm{T}} \in \mathcal{C}^{K \times 1}$ is the data symbol vector. Subsequently, the signal for user $k$ at the receiver side can be expressed as [6]

$$
y_{k}=\mathbf{h}_{k}^{\mathrm{T}} \mathbf{x}+n_{k}=\mathbf{h}_{k}^{\mathrm{T}} \mathbf{W} \mathbf{s}+n_{k},
$$

where $y_{k}$ is the received signal for user $k, \mathbf{h}_{k} \in \mathcal{C}^{N_{\mathrm{T}} \times 1}$ is the channel vector between the BS and user $k$, and $n_{k}$ is the additive Gaussian noise with zero mean and variance $\sigma^{2}$ at the receiver side. (2) can also be written in a compact matrix form as

$$
\mathbf{y}=\mathbf{H W} \mathbf{s}+\mathbf{n},
$$

where $\mathbf{y} \in \mathcal{C}^{K \times 1}$ is the received signal vector, $\mathbf{H} \in \mathcal{C}^{K \times N_{\mathrm{T}}}$ is the concatenated channel matrix, and $\mathbf{n} \in \mathcal{C}^{K \times 1}$ is the additive noise vector.

Precoding approaches aims to design the precoding matrix $\mathbf{W}$ to achieve certain targets, which include linear closed-form precoding schemes such as MRT, ZF and RZF [3]-[5], nonlinear precoding schemes such as THP [22]-[24] and VP [25][27], and optimization-based precoding designs such as PM and SB [6]-[9], as already mentioned above. In this section, we briefly review ZF precoding as an illustrative example, which can be viewed as a special case of CI-based precoding, as shown later in Section III-F. To be more specific, the precoding matrix for $\mathrm{ZF}$ precoding can be expressed as [5]

$$
\mathbf{W}_{\mathrm{ZF}}=\frac{1}{f_{\mathrm{ZF}}} \cdot \mathbf{H}^{\mathrm{H}}\left(\mathbf{H H}^{\mathrm{H}}\right)^{-1},
$$

where $f_{\mathrm{ZF}}$ is the normalization factor that guarantees that the power of the transmit signal is not increased after precoding,

TABLE I: Notations

\begin{tabular}{|c|c|}
\hline$a$ & Scalar \\
$\mathbf{a}$ & Column vector \\
$\mathbf{A}$ & Matrix \\
$(\cdot)^{*}$ & Conjugate \\
$(\cdot)^{\mathrm{T}}$ & Transpose \\
$(\cdot)^{\mathrm{H}}$ & Conjugate transpose \\
$(\cdot)^{-1}$ & Inverse \\
$(\cdot)^{+}$ & Pseudo-inverse \\
$\operatorname{diag}(\cdot)$ & Transformation of a vector into a diagonal matrix \\
$\otimes$ & Kronecker product \\
$|\cdot|$ & Absolute value or modulus \\
$\|\cdot\|_{2}$ & $\ell_{2}$-norm \\
$\|\cdot\|_{\infty}$ & Uniform norm \\
$\Re\{\cdot\}$ & Extraction of the real part \\
$\Im\{\cdot\}$ & Extraction of the imaginary part \\
$\mathbf{I}_{K}$ & $K \times K$ identity matrix \\
$j$ & Imaginary unit \\
$\mathcal{C}^{n \times n}$ & Set of $n \times n$ complex-valued matrices \\
$\mathcal{R}^{n \times n}$ & Set of $n \times n$ real-valued matrices \\
card $\{\cdot\}$ & Cardinality of a set \\
\hline
\end{tabular}

which is also known as the noise amplification factor. For traditional $\mathrm{ZF}$ precoding, $f_{\mathrm{ZF}}$ is calculated as

$$
f_{\mathrm{ZF}}=\sqrt{\operatorname{tr}\left\{\left(\mathbf{H H}^{\mathrm{H}}\right)^{-1}\right\}}
$$

which is obtained based on the assumption of Gaussian signaling [5], where we note that the expression for $f_{\mathrm{ZF}}$ can be different if we normalize the precoded signal on a symbol level. By substituting (4) into (3), we obtain the received signal vector for $\mathrm{ZF}$ precoding as

$$
\mathbf{y}_{\mathrm{ZF}}=\frac{1}{f_{\mathrm{ZF}}} \cdot \mathbf{H H}^{\mathrm{H}}\left(\mathbf{H H}^{\mathrm{H}}\right)^{-1} \mathbf{s}+\mathbf{n}=\frac{1}{f_{\mathrm{ZF}}} \cdot \mathbf{s}+\mathbf{n},
$$

where we observe that $\mathrm{ZF}$ precoding forces the multi-user interference to be zero for each user, which is thus termed as ' $\mathrm{ZF}$ '.

For an arbitrary constellation, the received signal vector $\mathbf{y}$ needs to be further rescaled for correct demodulation, expressed as

$$
\mathbf{r}=\beta \cdot \mathbf{y}
$$

where $\mathbf{r}$ is the received symbol vector ready for demodulation, and $\beta$ is the rescaling factor. When closed-form precoding schemes such as $\mathrm{ZF}$ are adopted, $\beta$ is equal to the normalization factor included in the precoding matrix, i.e., $\beta=f_{\mathrm{ZF}}$ if the BS employs ZF precoding. On the other hand, when optimization-based precoding approaches are employed, $\beta$ is obtained by minimizing the MSE between the received and transmit symbol vector, which can be expressed in a closed form as [29]

$$
\beta=\frac{\Re\left(\mathbf{x}^{\mathrm{H}} \mathbf{H}^{\mathrm{H}} \mathbf{s}\right)}{\|\mathbf{H} \mathbf{x}\|_{2}^{2}+K \sigma^{2}},
$$

We note that the above rescaling operation (7) is not necessarily required for PSK constellations, since it is sufficient to demodulate the received symbols based on their phases when a PSK modulation is adopted. We refer the interested readers to the overview papers [28], [30] and [31] for a more detailed description on precoding techniques.

In what follows, we introduce CI and interference exploitation techniques by first illustrating a simple example that characterizes $\mathrm{CI}$, as detailed below.

\section{B. Interference in Wireless Communications - Is It All Harm- ful?}

Traditionally, interference is usually viewed as a performance limiting factor in wireless communication systems. In a typical multi-user transmission, the existence of interference is based on the observation that the transmit signals for different users are superimposed in wireless communication channels. Precoding strategies are designed based on the fact that, with CSI known at the BS and potentially with the information of the data symbols as well, multi-user interference is able to be predicted prior to transmission. In fact, information theoretical analysis in [2] shows that when CSI is available at the transmitter, known interference will not affect the capacity of the broadcast channel. More specifically, the DPC method implies that it is optimal to code along interference, instead of attempting to mitigate or cancel interference. Nevertheless, the 
majority of existing linear precoding schemes still aim to eliminate, avoid or limit the interference [3]-[9]. In these traditional precoding schemes, the precoding matrix is designed based on the CSI only and therefore operate on a block level. In other words, the same precoding matrix is applied across a block of symbols and is updated when the channel changes. This means that only the power of the interference can be controlled, which leads to the statistical view that the effect of interference is similar to noise. On the other hand, if we observe interference from an instantaneous instead of statistical point of view, recent studies have shown that CI precoding via SLP is able to control both the power and the direction of the interfering signals on the received complex plane on a symbol level, such that the interference can act as an additional source of the desired signal power and contribute to the symbol detection, which therefore further improves the system performance [32], [33]. Based on the above description, interference exploitation techniques are foreseen to be most useful in systems where interference can be predicted and manipulated. To motivate the exploitation of interference in precoding designs, we firstly present illustrative examples to demonstrate how instantaneous interference can be divided into CI and destructive interference (DI) below, followed by the systematic CI characterization in Section II.

Let's first consider a simple example where the desired symbol $u$ is from a nominal BPSK constellation [34], and without loss of generality we assume $u=1$. We express the received signal as [35]

$$
y=u+i+n=r+n,
$$

where $i$ is the interfering signal, $r$ denotes the received signal excluding noise, and $n$ denotes the additive noise at the receiver side. We consider two distinct cases: (i) $i>0$ and (ii) $i<0$. When $i>0$, the resulting noiseless received signal $r>1$, which means that the interference has pushed $r$ further away from the detection threshold of BPSK, compared with the original data symbol $u$. In this case, the interfering signal $i$ contributes to the useful signal power and is in fact 'constructive'. Given a fixed noise power, $y=u+i+n$ is more likely to be correctly detected than the interferencefree case $\tilde{y}=u+n$, and an improved performance can be expected. On the other hand, when $i<0$, the interfering signal causes the received signal $r$ to move closer to the detection threshold, where the interfering signal reduces the useful signal power and is therefore 'destructive'. In this case, the noiseless received signal $r=u+i$ is more vulnerable to noise than $\tilde{r}=u$.

The above examples have only considered the effect of interfering symbols. To make the concept of interference exploitation more explicit, in the following example we further take the effect of wireless channels into account. In this example, we consider a geometrical representation of an interference scenario with random channels, as shown in Fig. 2, where for simplicity we still assume that $u=1$ is the desired data symbol, $i=1$ is the data symbol from the interferer, $h_{u}$ denotes the wireless channel between the transmitter and the receiver, while $h_{i}$ and $\tilde{h}_{i}$ represent the channel between the interferer and the receiver for achieving

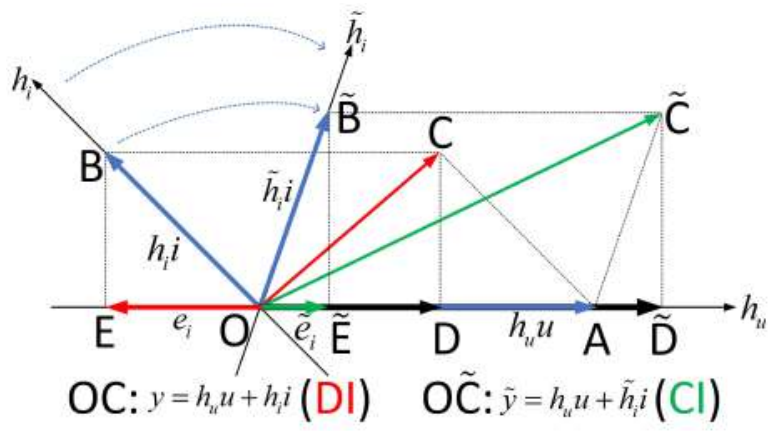

Fig. 2: The geometrical representation of CI and DI

DI and CI, respectively. Accordingly, the received signal can be expressed as [35]

$$
y_{\mathrm{DI}}=h_{u} u+h_{i} i, y_{\mathrm{CI}}=h_{u} u+\tilde{h}_{i} i,
$$

where we have assumed a noiseless case to focus on the effect of interference. In Fig. 2, DI is achieved when the channel between the interferer and the receiver is $h_{i}$. To be more specific, $\overrightarrow{O A}=h_{u} u$ is the useful signal while $\overrightarrow{O B}=h_{i} i$ is the interfering signal. $\overrightarrow{O C}=y_{\mathrm{DI}}$ is the received signal, and $\overrightarrow{O D}$ represents its projection on the axis of $h_{u}$, whose amplitude directly determines the detection performance. Geometrically, we observe that $|\overrightarrow{O D}|<|\overrightarrow{O A}|$, and consequently the interference is destructive since it reduces the useful signal power. To take a closer look, we can project the interfering signal $\overrightarrow{O B}$ onto the axis of $h_{u}$, and it is then observed that the direction of the effective interfering signal $\overrightarrow{O E}=e_{i}$ is to the opposite side of the desired signal $\overrightarrow{O A}$, which is similar to the case of (ii) $i<0$ discussed in the previous example and results in DI. On the other hand, when the interfering channel is shifted from $h_{i}$ to $\tilde{h}_{i}$, the interfering signal becomes constructive to the desired data symbol $u$, since they add up constructively and yield a received symbol whose amplitude is larger than that of the original transmit signal, as shown in Fig. 2 where $|\overrightarrow{O D}|>|\overrightarrow{O A}|$. This can also be observed from the fact that $\overrightarrow{O \tilde{E}}=\tilde{e}_{i}$, which is the projection of the interfering signal $\overrightarrow{O \tilde{B}}$ onto $h_{u}$, is in the same direction as the useful signal $\overrightarrow{O A}$, which is similar to the case of (i) $i>0$ discussed in the previous example and leads to CI. The above observation implies that for a given data symbol combination, some channel realizations may lead to CI while some other channel realizations may yield DI. Based on the above two simple examples, it is important to note that the classification of interference into constructive or destructive depends both on the data symbol combination and the CSI, as will be mathematically shown in Section II.

\section{CI Exploitation via Symbol-Level Precoding}

With the two examples illustrated above, we are now able to give the definition of CI: $\mathrm{CI}$ is the interference that pushes the received signals away from all of their corresponding decision boundaries of the modulated-symbol constellation, which thus 
contributes to the useful signal power ${ }^{1}$. Moreover, to exploit $\mathrm{CI}$ in the precoding design, firstly it should be highlighted that CI-based precoding has to be shifted from block-level operation to symbol-level operation, i.e., SLP is the method to achieve the CI effect. It should be noted that SLP is not limited as a method to exploit CI effects only, but also finds its application in hardware-efficient BS architecture, as will be discussed in Section V.

Early works on CI precoding techniques have focused on the adaptation of simple linear precoding methods such as ZF and RZF for CI exploitation [36]-[38]. In [36] and [37], for the first time the instantaneous interference in a MIMO system is characterized and classified into CI and DI, and a selective precoding is proposed where the $\mathrm{CI}$ is retained while the DI is cancelled via ZF. A more advanced approach termed correlation-rotation precoding is proposed in [38], where instead of being cancelled as in [36] and [37], the DI is manipulated and further rotated to be aligned with the desired data symbols such that DI becomes CI. Compared with the selective precoding in [36] and [37] that exploits interference only when it is constructive, the correlation-rotation precoding proposed in [38] directly controls interference such that all the interference for each user becomes constructive in the system.

The concept of CI has subsequently been applied to the non-linear THP method in [23], [24] and VP precoding in [39]. The interference-optimized THP (IO-THP) proposed in [23] introduces a complex scaling to the first user such that the interfering signals are better aligned to the symbols of interest, and by optimizing the complex scaling factor to minimize the power of the modified transmit signals, IO-THP reduces the power loss of the conventional THP schemes. As a step further, the power-efficient THP (PE-THP) method proposed in [24] allows complex scaling for a number of users, instead of for the first user only as in [23]. Compared with IO-THP in [23], the performance improvements come from the fact that PE-THP allows a larger number of variables to be optimized jointly within the constructive area and the signal-to-noise ratio (SNR) threshold, which generally leads to a better and more power-efficient THP solution. [39] proposes CI techniques in the context of VP precoding by substituting the search for the perturbation vectors with a linear scaling precoder, which removes the sophisticated sphere-search process and is the first optimization-based CI technique that involves a linear symbolscaling operation based on quadratic programming (QP).

More recently, CI-based precoding techniques have been widely combined with optimization to achieve further performance improvements [40]-[46]. In [41] and [42], the authors firstly propose a CI-MRT precoding method that improves the performance of correlation-rotation precoding by avoiding the ZF operation. In addition, PM optimization and weighted SB optimization based on the same CI metric are further discussed in [42]. It is worth mentioning that for CI precoding designs in [41] and [42], the received signals are forced to be strictly aligned to the desired data symbols with an increase in the amplitude for achieving CI, which follows the CI metric in

\footnotetext{
${ }^{1}$ Based on this definition, for multi-level modulations only the outer constellation points can exploit CI, which will be discussed later in Section II-B.
}

[38] and is later shown to be sub-optimal and termed 'strict phase rotation' in [58] (Fig. 4a). A more advanced CI metric is introduced in [43] and [44], where the concept of 'constructive region' is characterized for PSK constellations, within which all the interference is shown to be constructive. This relaxed CI metric reveals that it is no longer necessary for the interfering signals to be strictly aligned to the symbols of interest, which leads to further performance gains and is superior to the 'strict phase-rotation' CI metric in [38], [41] and [42]. This advanced CI metric is later termed 'non-strict phase rotation' in [58] (Fig. 4b), and is widely adopted in the subsequent precoding designs for CI exploitation [51], [52], [58]-[63] and its applications. Meanwhile, a similar and sub-optimal relaxed CI metric is also presented in [45], [46], where the 'relaxed detection region' metric that is determined by a phase margin related to the SNR target is introduced.

It should be noted that the above works [23], [24], [36][38], and [41]-[46] have all focused on PSK constellations for CI precoding based on the 'phase-rotation' metric, which is not applicable to QAM modulations since only the real or imaginary part of some constellation points from QAM modulation can exploit CI (Fig. 3). To this end, the extension to multi-level modulations such as QAM has recently been discussed in [47], [48] and [59]-[64], where the 'symbolscaling' CI metric is adopted. Interestingly, in contrast to claims that CI precoding may not be promising for higherorder QAM modulations since only the outer constellation points benefit from CI, [60] shows that substantial gains can still be observed even for a 64QAM constellation, which will also be numerically shown in Section III. This is because CI exploitation precoding not only allows the outer constellation points to benefit from CI, but more importantly also reduces the noise amplification effect, which is more prominent for a high-order QAM modulation. CI precoding has further been extended to generic two-dimension constellations with any shape and size in [56], where the CI metric is termed 'distance preserving CI region' (DPCIR). Additional studies on CI precoding include per-antenna power constraint [49], MMSEbased CI [50], noise-robust CI [51], symbol error rate (SER) minimization [52], non-linear channels [53], CI for generic constellations [54], multi-group multicasting [65], closed-form and iterative CI solutions [57]-[62], etc., and we summarize the major research outputs on CI precoding in Table II.

Meanwhile, a similar concept coined as 'directional modulation' [66]-[68], which was studied in the past in the context of analog RF and antenna components, has also emerged as a promising hardware-efficient approach, where the phase and amplitude of the transmitting signal on each antenna are directly designed such that multiple interference-free or interference-limited symbols can be transmitted to the receiver, which will be discussed in more detail in Section V-F.

\section{Benefits of CI and Symbol-Level Precoding}

With the ability to transform the power of the interfering signals into useful signal power without the need of investing additional transmit power, CI precoding has become an appealing PHY technique for wireless communications. Since future 
TABLE II: A summary for CI precoding techniques in a typical multi-user downlink transmission

\begin{tabular}{|c|c|c|c|}
\hline Reference & Considered Problem & CI Metric & Considered Modulation \\
\hline [37] & Selective CI & Strict phase rotation & PSK \\
\hline [38] & Correlation-rotation $\mathrm{CI}$ & Strict phase rotation & PSK \\
\hline [39] & CI-VP & Symbol scaling & PSK \\
\hline [40] & CI-based SB & Non-strict phase rotation & PSK + QAM \\
\hline [41], [42] & CI-MRT + PM + weighted SB & Strict phase rotation & PSK \\
\hline [43], [44] & CI-based $\mathrm{PM}+\mathrm{SB}$ & Non-strict phase rotation & PSK \\
\hline [45], [46] & CI-based PM + weighted SB & Relaxed detection region & PSK \\
\hline [47], [48] & CI-based PM & Symbol scaling & QAM \\
\hline [49] & CI-based Per-antenna PM & Strict phase rotation & PSK \\
\hline [50] & CI-MMSE precoding & Non-strict phase rotation & PSK \\
\hline$[51],[52]$ & CI-based $\mathrm{PM}+\mathrm{SB}$ & Noise-robust $\mathrm{CI}$ & PSK \\
\hline [53] & CI-based weighted per-antenna PM & Strict phase rotation & APSK \\
\hline [54], [55], [56], [57] & CI-based PM + SB & Distance preserving CI region & Any constellation \\
\hline$[58],[59],[60],[61],[62]$ & Closed-form CI solutions & Non-strict phase rotation + Symbol scaling & PSK + QAM \\
\hline
\end{tabular}

cellular communication systems are foreseen to be deployed in a heterogeneous form, where macro BSs employ hardwareefficient large-scale antenna arrays for both cost efficiency and energy efficiency, while small BSs employ fully-digital smallscale antenna arrays for spectral efficiency. For fully-digital small-scale antenna arrays, using CI-based SLP techniques can greatly enhance the link performance to meet the increasing throughput requirement for future wireless systems, as will be discussed and numerically shown in Section III. Meanwhile, when hardware-efficient large-scale arrays are adopted by macro BSs, CI-based SLP techniques will also be required in order to achieve a satisfactory performance, since traditional precoding techniques usually do not perform well in such hardware-constrained scenarios, as will be discussed in Section $\mathrm{V}$.

Obviously, the most prominent advantage for CI precoding over conventional precoding is the significant performance improvements in terms of error rate performance and transmit power savings. By carefully designing the precoding matrix based on the CSI as well as information of the data symbols, interference that is usually regarded as a detrimental effect and needs to be mitigated now becomes beneficial and further contributes to the increase in the useful signal power [44] which has also been mathematically studied in [48] using an equivalent PHY multicasting model. In addition to the above most significant advantage, some additional benefits that CI precoding enjoys should also be highlighted in particular.

1) Instantaneous Constraints: Conventional precoding methods operate on a block level, and the constraints such as the SINR target for the PM problems or the transmit power requirement for the SB optimizations are met averaged over a transmission block. While these constraints are indeed satisfied from a statistical point of view, during actual transmission these constraints may be violated for some data symbol combinations while over-satisfied for other data symbol combinations within each transmission block.
As a comparison, CI precoding does not have this issue and guarantees that the constraint is strictly satisfied for each data symbol combination during transmission, because CI precoding works on a symbol level and accordingly the constraints are enforced on a symbol level. This can be mathematically observed in [44] as well as in Section III for optimization problems formulated based on CI precoding, where the data symbols have been included in the constraints.

2) Receiver Complexity: CI precoding provides additional complexity reduction at the receiver side. When traditional block-level optimization-based PM or SB precoding is adopted, since the optimization only focuses on the power of the useful signals without considering the phase-rotation effect, each receiver therefore needs to perform phase estimation and compensate the phase-rotation effect of the channel, before the symbols can be correctly decoded, which may suffer from channel estimation errors [35].

In contrast to these traditional precoding strategies, the received symbols for CI precoding are all located in the constructive area, and therefore the phase-compensation process is not required any more, when PSK constellations are employed. This means that the symbol decoding process does not require the estimated CSI at the receivers, as only a simple decision stage is sufficient, which therefore avoids the effect of CSI estimation errors on the decoding process. This is particularly important in the case of downlink transmission where the receivers are typically computationally constrained mobile devices. Accordingly, CI precoding approaches may further lead to savings in the training time and overhead for signaling the composite channels to the receivers, as demonstrated in [35].

3) Data Stream Number Increase: In addition to the above two benefits, another benefit that is recently revealed for CIbased precoding over traditional precoding is the support for an increased number of data symbols that can be simultaneously transmitted [44], [59], [60]. For traditional precoding 


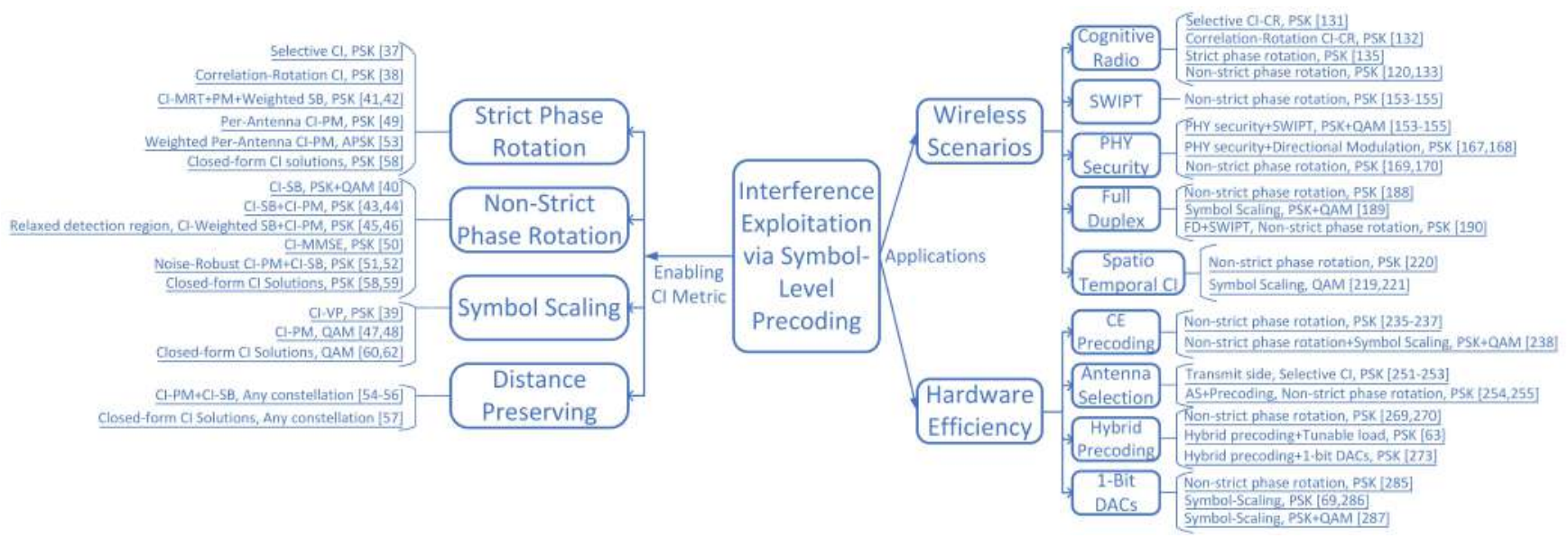

Fig. 3: Classification of CI Metrics and Applications of Symbol-Level Precoding

approaches, the number of data streams that can be supported for simultaneous transmission is limited by the number of transmit antennas at the BS in the downlink. While MRT and RZF precoding can theoretically support a larger number of data streams, the resulting uncoded bit error rate (BER) performance is on the order of $10^{-1}$, which is not practically useful. For CI precoding, on the contrary, it is shown in [59], [60] that it can support a larger number of data streams with a significantly improved BER performance. Specifically, when there are $N_{t}=8$ transmit antennas at the BS and a total number of $K=9$ users in the system, CI precoding is able to achieve an uncoded BER that is lower than $10^{-4}$ when QPSK modulation is considered [59]. A similar result can be observed in [60] for QAM constellations, while the supported number of data streams becomes fewer compared with PSK modulations.

In addition to the above general benefits, a number of application-particular benefits have also been reported and will be overviewed in the respective sections in the following.

\section{E. Current Limitations of CI and Symbol-Level Precoding}

In addition to various benefits CI precoding offers compared with traditional channel-dependent precoders, CI precoding also exhibits some limitations, as discussed below.

1) Complexity: The first and most obvious limitation for CI precoding is its complexity, which also holds for other SLP-based techniques. Compared with traditional block-level precoders where the precoding matrix is updated whenever the channel changes, CI precoding has to update the precoding matrix on a symbol level, which leads to much higher computational costs. Moreover, most CI precoding approaches in the literature are based on optimizations, which means that an optimization problem has to be solved to obtain the desired precoding matrix on a symbol level, which is more demanding than block-level precoders. Both of the above requirements pose significant burden on the hardware components at the BS, especially in practical wireless communication systems that are required to operate on a real-time basis.

Nevertheless, thanks to recent developments in deriving low-complexity solvers for CI precoding, it has been shown in [58] and [60] that an optimal CI precoding structure exists for the CI-based max-min fair optimization problem, while [57] also presents a closed-form expression for CI-based PM problem by following a similar approach, both of which can greatly alleviate the computational costs for CI precoding. This will be discussed in more detail in Section III-F.

2) CI based on Soft Detector: Another limitation for current CI-based SLP schemes is that they are designed for uncoded communication systems, and its superiority is therefore only guaranteed for uncoded systems. In practical wireless communication systems where channel coding is also adopted, it is shown in [66] that CI precoding with existing channel coding schemes is superior to traditional channel-coded precoding schemes. However, it is still unknown whether this is the optimal performance and whether a joint design of CI precoding and channel coding can lead to further performance improvements. To design the CI-optimal soft detector, the priori probabilities of the input symbols producing a soft output indicating the reliability of the decision should be taken into account, which have not been fully addressed in the literature.

3) Adaptive Modulation and SINR Estimation: Current CI precoding approaches are designed for either PSK or QAM constellations, while the extension to a multiple modulation scenario has not been fully discussed. Importantly, since practical wireless communication systems adopt adaptive modulation based on SINR estimation, current CI precoding algorithms designed for single modulation are not directly applicable. Moreover, SINR estimation techniques currently employed may not be suitable for CI-based precoding methods. This will be discussed in Section VII-C.

In the following, we firstly characterize the CI metric, based on which optimization-based CI precoding techniques are discussed, followed by the extension to a variety of wireless scenarios and hardware efficiency, as depicted in Fig. 3.

\section{Constructive Interference Classification AND CHARACTERIZATION}

In this section, we characterize the CI condition for several modulations that are typically considered in wireless com- 

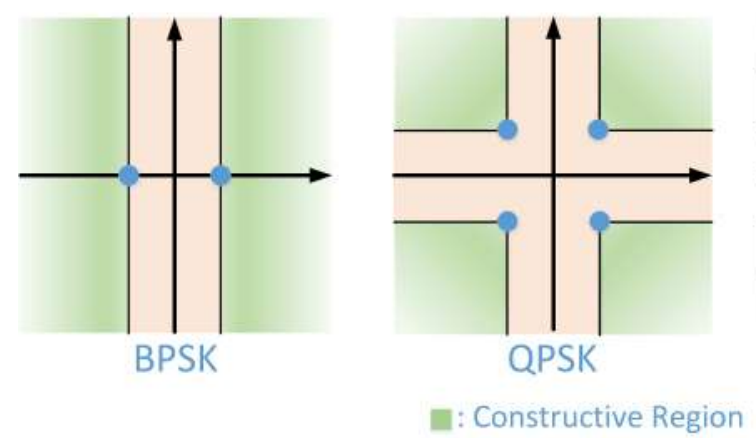

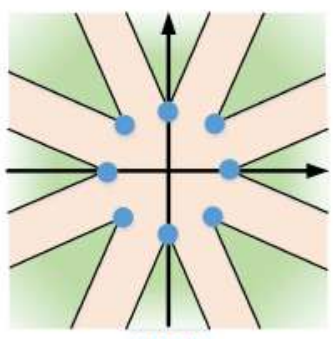

8PSK

: Destructive Region

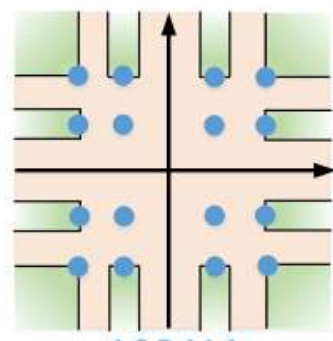

16QAM

Fig. 4: CI region characterization for PSK and QAM constellations

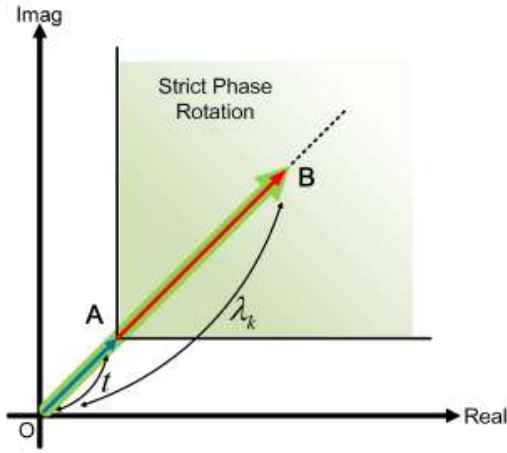

(a) QPSK, 'strict', [58]

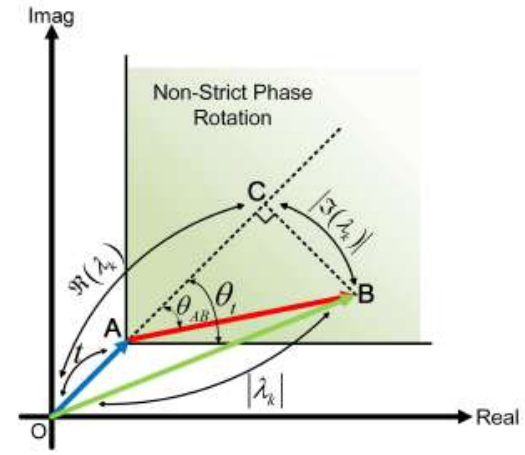

(b) QPSK, 'non-strict', [58]

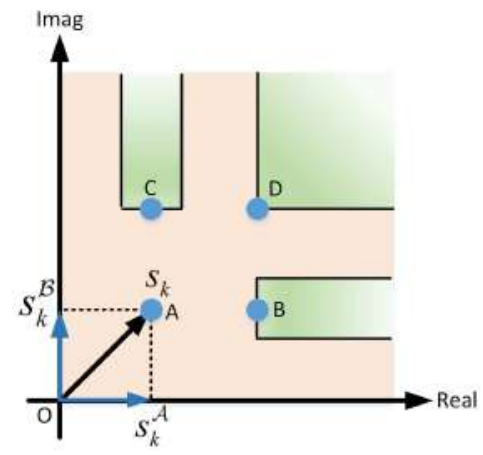

(c) QAM, 'symbol-scaling', [60]

Fig. 5: CI metric for PSK based on 'strict/non-strict phase rotation' and QAM based on 'symbol scaling'

munication systems, which include PSK, QAM, and APSK constellations. For each modulation type, we first illustrate the CI condition geometrically, based on which we present the mathematical condition for achieving CI. While there also exist several alternative CI metrics in the literature [44], [46], [54], and [56], in this paper we employ the CI metric that is firstly introduced in [44] and widely adopted in the subsequent studies for CI-based precoding. Throughout this section, we consider unit-norm constellations to characterize the CI condition for each modulation type.

Based on the definition of CI and following [44], [60], the CI regions for PSK and QAM constellations are shown as the green shaded areas in Fig. 4, where BPSK, QPSK, 8PSK and 16QAM constellations are depicted as representative examples, and their extensions to higher PSK and QAM constellations are trivial. As can be seen, when the received signal is located in the green 'constructive region', its distance to all the detection thresholds is increased compared with the nominal constellation point.

\section{A. Constant-Envelope Constellations (PSK)}

To derive the mathematical formulation of CI conditions for constant-envelope PSK constellations, we consider the 'phase-rotation' metric introduced in [44], and without loss of generality we focus on one quarter of a nominal QPSK constellation, as depicted in Fig. 5a for 'strict phase rotation' and Fig. 5b for 'non-strict phase rotation', where $\overrightarrow{O B}$ denotes the noiseless received signal, which leads to $\overrightarrow{O B}=\mathbf{h}_{k}^{\mathrm{T}} \mathbf{W} \mathbf{s}$. $\overrightarrow{O A}=t \cdot s_{k}$ represents the scaled data symbol, and $t=\sqrt{\Gamma_{k} \sigma^{2}}$ for PM optimizations, where $\Gamma_{k}$ is the corresponding SINR target for user $k$ [44]. For SB optimizations, $t$ is the objective function to be maximized [58]. Based on the observation that $\overrightarrow{O B}=\overrightarrow{O A}+\overrightarrow{A B}$, we obtain that $\overrightarrow{A B}=\mathbf{h}_{k}^{\mathrm{T}} \mathbf{W} \mathbf{s}-t \cdot s_{k}$ is the interfering signals. For notational convenience, we introduce a scalar $\lambda_{k}$ for each data symbol $s_{k}$, which leads to [58]

$$
\overrightarrow{O B}=\mathbf{h}_{k}^{\mathrm{T}} \mathbf{W} \mathbf{s}=\lambda_{k} s_{k},
$$

and the value of $\lambda_{k}$ fully represents the effect of multi-user interference.

For the 'strict phase-rotation' CI metric as considered in [41], [42] and [58], it is obvious that each $\lambda_{k}$ should be purely real to guarantee that the phase of the noiseless received signal for user $k$ is exactly the same as that of $s_{k}$. Furthermore, the received signals should enhance the useful signal power to achieve CI, and therefore the mathematical condition for the 'strict phase-rotation' metric can be expressed as [58]

$$
\lambda_{k} \geq t, \forall k \in \mathcal{K}
$$

where $\mathcal{K}=\{1,2, \cdots, K\}$.

For the 'non-strict phase-rotation' metric proposed in [44], $\lambda_{k}$ can be complex as the phases of the received signals are not necessarily constrained to be strictly aligned to the original symbols of interest. As observed in Fig. 5b, as long as the resulting signals are located in the 'constructive region', all the interference is constructive and further benefits the detection 
performance. Based on the geometry, to have the node ' $\mathrm{B}$ ' located in the constructive area is equivalent to [58]

$$
\theta_{A B} \leq \theta_{\mathrm{th}}
$$

where $\theta_{\text {th }}=\frac{\pi}{\mathcal{M}}$ for a $\mathcal{M}$-PSK constellation. This CI condition in (13) can be further expressed as a function of the complex scalar $\lambda_{k}$, given by [44], [58]

$$
\left[\Re\left(\lambda_{k}\right)-t\right] \tan \theta_{\text {th }} \geq\left|\Im\left(\lambda_{k}\right)\right|, \forall k \in \mathcal{K},
$$

which is widely employed in the literature of CI-based precoding [43], [44], [51], [52], [58]-[63]. Specifically for a BPSK constellation where $\mathcal{M}=2$, the CI condition in (14) for the 'non-strict phase-rotation' metric is simplified into

$$
\Re\left(\lambda_{k}\right)-t \geq 0, \forall k \in \mathcal{K} .
$$

\section{B. Multi-Level Constellations (QAM, APSK)}

In this section, we describe the CI condition for multilevel modulations, where we consider QAM and APSK as two representative examples. The general observation of CI characterization for multi-level modulations is that $\mathrm{CI}$ can be exploited by the outer constellation points, while we consider all the interference for the inner constellation points as destructive. Therefore, the CI condition for multi-level modulations is formulated by decomposing the constellation points into outer constellation points and inner constellation points, as detailed below.

1) QAM: QAM modulation is a typical multi-level modulation widely employed in wireless communication systems. CIbased precoding for QAM constellations is firstly considered in [47], [48], [63] and [64], and is also studied in [60] more recently. For QAM modulation, since only the real (or imaginary) part of some outer constellation points can be scaled to exploit CI, as observed in Fig. 4 and Fig. 5c, the 'phase-rotation' metric in [41]-[44] is no longer applicable to QAM.

Accordingly, the 'symbol-scaling' metric considered in [48], [60] and [63] is presented here. To be more specific, this metric first performs a signal decomposition of the constellation points as well as the noiseless received signals along the detection thresholds, which mathematically leads to [60], [69]

$$
s_{k}=s_{k}^{\mathcal{A}}+s_{k}^{\mathcal{B}}, \mathbf{h}_{k}^{\mathrm{T}} \mathbf{W} \mathbf{s}=\alpha_{k}^{\mathcal{A}} s_{k}^{\mathcal{A}}+\alpha_{k}^{\mathcal{B}} s_{k}^{\mathcal{B}}=\mathbf{\Omega}_{k}^{\mathrm{T}} \mathbf{s}_{k}, \forall k \in \mathcal{K} .
$$

where $s_{k}^{\mathcal{A}}$ and $s_{k}^{\mathcal{B}}$ are parallel to the two detection thresholds for the constellation point $s_{k}, \boldsymbol{\Omega}_{k}=\left[\alpha_{k}^{\mathcal{A}}, \alpha_{k}^{\mathcal{B}}\right]^{\mathrm{T}}$ and $\mathbf{s}_{k}=$ $\left[s_{k}^{\mathcal{A}}, s_{k}^{\mathcal{B}}\right]^{\mathrm{T}}$. In (16), $\alpha_{k}^{\mathcal{A}} \geq 0$ and $\alpha_{k}^{\mathcal{B}} \geq 0$ are two real scalars that jointly represent the effect of interference. Specifically for QAM constellations, we can directly simplify the expression for $s_{k}^{\mathcal{A}}$ and $s_{k}^{\mathcal{B}}$ into [60]

$$
s_{k}^{\mathcal{A}}=\Re\left\{s_{k}\right\}, s_{k}^{\mathcal{B}}=j \cdot \Im\left\{s_{k}\right\} .
$$

For notational convenience, we introduce a set $\mathcal{O}$ that consists of the real scalars corresponding to the real (or imaginary) part of the outer constellation points that can be scaled to exploit $\mathrm{CI}$, and a set $\mathcal{I}$ that consists of the real scalars that correspond to the real (or imaginary) part of the constellation points that cannot be scaled [60], i.e., in Fig. 5c, the set $\mathcal{O}$ includes the scalars for both the real and imaginary part of the constellation point type ' $D$ ', the real part of ' $B$ ', and the imaginary part of ' $C$ '. Similarly, the set $\mathcal{I}$ includes the scalars for both the real and imaginary part of the constellation point type 'A', the real part of ' $C$ ', and the imaginary part of 'B'. Accordingly, the set $\mathcal{O} \cup \mathcal{I}$ includes all the scaling factors $\alpha_{k}^{\mathcal{U}}, \mathcal{U} \in\{\mathcal{A}, \mathcal{B}\}, \forall k \in \mathcal{K}$, and the CI condition for QAM constellations can be expressed as [60]

$$
\alpha_{m}^{\mathcal{O}} \geq t, \alpha_{n}^{\mathcal{I}}=t, \forall \alpha_{m}^{\mathcal{O}} \in \mathcal{O}, \forall \alpha_{n}^{\mathcal{I}} \in \mathcal{I}
$$

Similar to the case of PSK modulation, $t$ is the objective function for SB optimizations, while $t=\sqrt{\Gamma_{k} \sigma^{2}}$ if a PM problem is considered.

It should be noted that while the 'symbol-scaling' metric is primarily considered for QAM constellations, it is applicable to PSK modulation as well, as firstly studied in [39] in the context of VP precoding. Nevertheless, for a generic PSK constellation, the expression for $s_{k}^{\mathcal{A}}$ and $s_{k}^{\mathcal{B}}$ is no longer in the form in (17), and the corresponding derivations can be found in [69] in detail.

2) APSK: APSK is another representative example of multi-level modulation, which has been widely considered in satellite communications [70], [71]. Compared with QAM constellations, the advantage for APSK modulation lies in its low peak-to-average power ratio (PAPR) and is therefore more robust against nonlinear channel effects. The constellation of APSK can be viewed as a combination of several PSK constellations with different amplitudes [70].

Similar to the case of QAM, CI can be exploited by the outer constellation points of APSK, while the inner constellation points should not be scaled. Since the outer constellation of APSK can be regarded as a typical PSK constellation, by following (11), (13) and (14), the CI condition for APSK can be readily expressed as

$$
\left[\Re\left(\lambda_{m}^{\mathcal{O}}\right)-t\right] \tan \theta_{\text {th }} \geq\left|\Im\left(\lambda_{m}^{\mathcal{O}}\right)\right|, \lambda_{n}^{\mathcal{I}}=t,
$$

TABLE III: A summary of mathematical CI conditions for PSK and multi-level modulation

\begin{tabular}{|c|c|c|c|}
\hline Modulation & CI Metric & Signal Representation & Mathematical CI Condition \\
\hline PSK & Strict Phase Rotation [42], [58] & $\mathbf{h}_{k}^{\mathrm{T}} \mathbf{W} \mathbf{s}=\lambda_{k} s_{k}$ & $\lambda_{k} \geq t$ \\
\hline PSK & Non-Strict Phase Rotation [44], [58] & $\mathbf{h}_{k}^{\mathrm{T}} \mathbf{W} \mathbf{s}=\lambda_{k} s_{k}$ & {$\left[\Re\left(\lambda_{k}\right)-t\right] \tan \theta_{\mathrm{th}} \geq\left|\Im\left(\lambda_{k}\right)\right|$} \\
\hline PSK & Symbol Scaling [39], [69] & $\mathbf{h}_{k}^{\mathrm{T}} \mathbf{W s}=\alpha_{k}^{\mathcal{A}} s_{k}^{\mathcal{A}}+\alpha_{k}^{\mathcal{B}} s_{k}^{\mathcal{B}}$ & $\alpha_{k}^{\mathcal{A}} \geq t, \alpha_{k}^{\mathcal{B}} \geq t$ \\
\hline QAM & Symbol Scaling [60] & $\mathbf{h}_{k}^{\mathrm{T}} \mathbf{W} \mathbf{s}=\alpha_{k}^{\mathcal{A}} s_{k}^{\mathcal{A}}+\alpha_{k}^{\mathcal{B}} s_{k}^{\mathcal{B}}$ & $\alpha_{m}^{\mathcal{O}} \geq t, \alpha_{n}^{\mathcal{I}}=t$ \\
\hline APSK & Non-Strict Phase Rotation & $\mathbf{h}_{k}^{\mathrm{T}} \mathbf{W} \mathbf{s}=\lambda_{k} s_{k}$ & {$\left[\Re\left(\lambda_{m}^{\mathcal{O}}\right)-t\right] \tan \theta_{\mathrm{th}} \geq\left|\Im\left(\lambda_{m}^{\mathcal{O}}\right)\right|, \lambda_{n}^{\mathcal{I}}=t$} \\
\hline
\end{tabular}


where $\theta_{\text {th }}=\frac{\pi}{\mathcal{N}}$ when the outer ring of the APSK constellation adopts $\mathcal{N}$-PSK, $\lambda_{m}^{\mathcal{O}} \in \mathcal{O}$, and $\lambda_{n}^{\mathcal{I}} \in \mathcal{I}$. In the case of APSK, the set $\mathcal{O}$ includes the complex scalars corresponding to the outer constellation points of APSK, while $\mathcal{I}$ includes the complex scalars corresponding to the inner constellation points, respectively, and we have $\mathcal{O} \cup \mathcal{I}=\left\{\lambda_{1}, \lambda_{2}, \cdots, \lambda_{K}\right\}$.

We summarize the mathematical CI formulation for the discussed modulation types in Table III.

\section{Symbol-Level Precoding BASED ON OPTIMIZATION}

In this section, we introduce several optimization-based CI precoding designs in the downlink of a MU-MISO system, which includes CI-based VP precoding, CI-based PM, CIbased $\mathrm{SB}^{2}$, etc., as a foundation for the description of the applications of interference exploitation in Section IV and V. In each subsection, we begin with a brief literature review on these precoding approaches and their corresponding mathematical formulations, followed by their adaptations to CIbased designs.

\section{A. CI-VP based on Symbol Scaling}

Traditional non-linear VP approaches apply a perturbation to the data symbols upon the ZF precoding [25]-[27], such that the resulting transmit signals are better aligned to the eigenvectors of the channel inverse matrix, which leads to a significant decrease in the noise amplification effect. Accordingly, a significantly improved error rate performance is observed for VP precoding over ZF precoding in the high SNR regimes. Meanwhile, since VP approach is highly computationally expensive due to the inclusion of the sphere-search process, a number of studies on VP precoding have focused on the reduction in the computational costs [72]-[74]. Specifically, this includes the thresholded VP method where the spheresearch process is terminated when a SNR target is met [72], [73], and the selective VP approach that only perturbs part of the data symbols for maximizing the energy efficiency [74]. In addition to complexity reduction, several studies have also paid their attention to the modulo loss reduction for VP precoding [75]-[77]. Meanwhile, an additional problem for conventional VP precoding in [25] is that it does not directly apply to multimodulation scenarios, since the perturbation basis is modulation dependent [25]. To overcome this, several studies have considered multi-modulation VP precoding, which includes the block-diagonalized VP method in [78], the user-grouping VP method in [79], and a more superior constellation scaling approach in [26], [27].

The precoded signal vector for a traditional VP precoding can be expressed as [25]

$$
\mathbf{x}_{\mathrm{VP}}=\frac{\sqrt{P_{0}}}{\beta_{\mathrm{VP}}} \cdot \mathbf{H}^{\mathrm{H}}\left(\mathbf{H H}^{\mathrm{H}}\right)^{-1}(\mathbf{s}+\tau \cdot \mathbf{l}),
$$

\footnotetext{
${ }^{2}$ The optimization for the sum-rate maximization is not considered, since the Shannon sum-rate expression is not applicable to CI precoding. This will be discussed in Section VII-A.
}

where $P_{0}$ is the transmit power, $\tau$ is the modulation-dependent modulo basis, $l$ is the complex-integer perturbation vector, and

$$
\beta_{\mathrm{VP}}=\left\|\mathbf{H}^{\mathrm{H}}\left(\mathbf{H H}^{\mathrm{H}}\right)^{-1}(\mathbf{s}+\tau \cdot \mathbf{l})\right\|_{2}
$$

is the scaling factor for power normalization, which is also known as the noise amplification factor [25]. For conventional VP precoding, the perturbation vector $\mathbf{l}$ is found by the sphere-search algorithm in the whole complex-integer space to minimize the power normalization factor $\beta_{\mathrm{VP}}$, and a modulo operation with basis $\tau$ is also required at the receiver side to remove the perturbation effect.

CI-based VP approach is proposed in the context of complexity reduction for VP precoding [39]. To extend the concept of CI exploitation to VP precoding, it is natural to constrain the search for the optimal perturbation vectors within the constructive area for each constellation point. This then removes the necessity for the modulo operation at the receiver side, since the perturbation vectors are all constructive and contribute to the original data symbols. Moreover, since the perturbation vectors are enforced in the constructive area, their values can be relaxed into complex continuous and are no longer needed to be complex integers. Accordingly, by introducing a diagonal scaling matrix $\boldsymbol{\Phi}$ with complex entries, the transmit signal vector for constructive VP (CVP) can be transformed into a linear scaling operation, expressed as [39]

$$
\mathbf{x}_{\mathrm{CVP}}=\frac{\sqrt{P_{0}}}{\beta_{\mathrm{CVP}}} \cdot \mathbf{H}^{\mathrm{H}}\left(\mathbf{H H}^{\mathrm{H}}\right)^{-1} \mathbf{\Phi} \mathbf{s},
$$

where the power scaling factor $\beta_{\mathrm{CVP}}$ is obtained as [39]

$$
\beta_{\mathrm{CVP}}=\left\|\mathbf{H}^{\mathrm{H}}\left(\mathbf{H H}^{\mathrm{H}}\right)^{-1} \mathbf{\Phi} \mathbf{s}\right\|_{2} .
$$

By following the decomposition in (16), we transform $\mathbf{\Phi}$ s into

$$
\mathbf{\Phi} \mathbf{S}=\mathbf{T} \operatorname{diag}\left\{\boldsymbol{\Phi}_{\mathbf{E}}\right\} \mathbf{S}_{\mathbf{E}}
$$

such that the scalars in $\boldsymbol{\Phi}_{\mathbf{E}}$ are real, where $\mathbf{T} \in \mathcal{R}^{K \times 2 K}$, $\mathbf{\Phi}_{\mathbf{E}} \in \mathcal{R}^{2 K \times 1}$ and $\mathbf{s}_{\mathbf{E}} \in \mathcal{C}^{2 K \times 1}$ are given by

$$
\begin{aligned}
& \mathbf{T}=\left[\begin{array}{cccccccc}
1 & 1 & 0 & 0 & \cdots & \cdots & 0 & 0 \\
0 & 0 & 1 & 1 & \ddots & \ddots & 0 & 0 \\
\vdots & \vdots & \ddots & \ddots & \ddots & \ddots & \vdots & \vdots \\
0 & 0 & \cdots & \cdots & 0 & 0 & 1 & 1
\end{array}\right]=\mathbf{I}_{K} \otimes[1,1] \\
& \mathbf{\Phi}_{\mathbf{E}}=\left[\alpha_{1}^{\mathcal{A}}, \alpha_{1}^{\mathcal{B}}, \alpha_{2}^{\mathcal{A}}, \alpha_{2}^{\mathcal{B}}, \cdots, \alpha_{K}^{\mathcal{A}}, \alpha_{K}^{\mathcal{B}}\right]^{\mathrm{T}} \\
& \mathbf{S}_{\mathbf{E}}=\left[s_{1}^{\mathcal{A}}, s_{1}^{\mathcal{B}}, s_{2}^{\mathcal{A}}, s_{2}^{\mathcal{B}}, \cdots, s_{K}^{\mathcal{A}}, s_{K}^{\mathcal{B}}\right]^{\mathrm{T}} .
\end{aligned}
$$

Accordingly, the optimization problem that minimizes the power scaling factor $\beta_{\mathrm{CVP}}$ based on CI exploitation is given by [39]

$$
\begin{array}{cc}
\mathcal{P}_{\mathrm{CVP}}: & \min _{\boldsymbol{\Phi}_{\mathbf{E}}}\left\|\mathbf{H}^{\mathrm{H}}\left(\mathbf{H H}^{\mathrm{H}}\right)^{-1} \mathbf{T} \operatorname{diag}\left\{\boldsymbol{\Phi}_{\mathbf{E}}\right\} \mathbf{s}_{\mathbf{E}}\right\|_{2}^{2} \\
\text { s.t. } & \mathrm{C} 1: \alpha_{k}^{\mathcal{U}} \geq \alpha_{0}, \mathcal{U} \in\{\mathcal{A}, \mathcal{B}\}, \forall k \in \mathcal{K}
\end{array}
$$

which can be further transformed into a QP and solved efficiently. In $\mathcal{P}_{\mathrm{CVP}}, \alpha_{0}>0$ is a lower threshold, whose value will not affect the final solution, and it is convenient to select $\alpha_{0}=1$. Compared with traditional VP precoding, the CVP method requires less than $10 \%$ the complexity when 
the number of antennas is larger than $K=N_{\mathrm{T}}=6$, by substituting the sophisticated sphere-search process with a linear scaling operation [39].

In addition to the formulation in (22), an alternative CI-VP approach has recently been presented in [80], where the data symbol vector $\mathbf{s}$ is replaced with an equivalent symbol vector $\tilde{\mathbf{s}}=[1,1, \cdots, 1] \in \mathcal{R}^{K \times 1}$, and the precoded signal vector is given by [80]

$$
\mathbf{x}_{\mathrm{CI}-\mathrm{VP}}=\frac{\sqrt{P_{0}}}{\beta_{\mathrm{CI}-\mathrm{VP}}} \mathbf{H}^{\mathrm{H}}\left(\mathbf{H} \mathbf{H}^{\mathrm{H}}\right)^{-1} \mathbf{B}(\tilde{\mathbf{s}}+\tilde{\mathbf{u}}),
$$

where $\tilde{\mathbf{u}}$ is the real non-negative perturbation vector and $\mathbf{B}$ is a rotation matrix defined as [80]

$$
\mathbf{B}=\left[\begin{array}{ccccc}
s_{1} & 0 & 0 & \cdots & 0 \\
0 & s_{2} & 0 & \cdots & 0 \\
0 & 0 & s_{3} & \cdots & 0 \\
\vdots & \vdots & \vdots & \ddots & \vdots \\
0 & \cdots & 0 & 0 & s_{K}
\end{array}\right] .
$$

The equivalent symbol vector $\tilde{\mathbf{s}}$ is real-valued rotated versions of the original complex data symbols. The rotation is accounted accordingly in (28) so that users still receive the correct symbols, since $\mathbf{B} \tilde{\mathbf{s}}=\mathbf{s}$. The power scaling factor is therefore calculated as

$$
\beta_{\mathrm{CI}-\mathrm{VP}}=\left\|\mathbf{H}^{\mathrm{H}}\left(\mathbf{H H}^{\mathrm{H}}\right)^{-1} \mathbf{B}(\tilde{\mathbf{s}}+\tilde{\mathbf{u}})\right\|_{2} .
$$

Consequently, the optimization problem can be constructed as [80]

$$
\begin{array}{cl}
\mathcal{P}_{\text {CI-VP }}: & \min _{\tilde{\mathbf{u}}}\left\|\mathbf{H}^{\mathrm{H}}\left(\mathbf{H} \mathbf{H}^{\mathrm{H}}\right)^{-1} \mathbf{B}(\tilde{\mathbf{s}}+\tilde{\mathbf{u}})\right\|_{2} \\
\text { s.t. } & \mathrm{C} 1: \tilde{u}_{k} \geq 0, \forall k \in \mathcal{K}
\end{array}
$$

The above optimization problem (30) is a non-negative least squares (NNLS) problem and can be solved directly using the Fast NNLS [81] or the closed-form algorithm given by [82], [83]. The introduction of the equivalent real symbol vector in this CI-VP approach allows to reduce the search space in the optimization problem and decrease its computational complexity by searching only for real-valued and non-negative perturbation vector.

\section{B. Power Minimization}

Traditional optimization-based PM problems aim to minimize the total transmit power subject to a minimum received SINR target at each receiver side [6]-[8], [84]-[86]. An uplinkdownlink duality has been revealed in [7] and [8], based on which an efficient algorithm is proposed to solve the downlink precoding optimization. Another method to solve PM optimizations is to transform the PM optimization into a semi-definite programming (SDP). Using the semi-definite relaxation (SDR) approach [84]-[86], the rank-relaxed SDP becomes a convex optimization that can be conveniently solved, and it has been proven that a rank-1 solution always exists when the problem is feasible [84]. A rank-reduction algorithm is further developed in [84] and [85] to effectively reduce the rank of the solution to the relaxed SDP problem, when additional shaping constraints are further included in the PM optimizations. It is further shown in [86] that the exploitation of real-valued orthogonal space time block coding (OSTBC) can effectively increase the degree of freedom in the optimization design, which leads an improved performance.

Mathematically, from a statistical point of view and treating interference as harmful, the SINR expression for user $k$ can be expressed as [6]

$$
\gamma_{k}=\frac{\left|\mathbf{h}_{k}^{\mathrm{T}} \mathbf{w}_{k}\right|^{2}}{\sum_{i \neq k}\left|\mathbf{h}_{k}^{\mathrm{T}} \mathbf{w}_{i}\right|^{2}+\sigma^{2}},
$$

which is obtained based on $\mathbb{E}\left\{\mathbf{s s}^{\mathrm{H}}\right\}=\mathbf{I}$. Given (31), a typical PM problem that targets at minimizing the average transmit power subject to the SINR threshold $\Gamma_{k}$ for user $k$ can be formulated as [6]

$$
\begin{aligned}
\mathcal{P}_{\mathrm{PM}}: & \min _{\mathbf{w}_{k}} \sum_{k=1}^{K}\left\|\mathbf{w}_{k}\right\|_{2}^{2} \\
\text { s.t. } & \mathrm{C} 1: \gamma_{k} \geq \Gamma_{k}, \forall k \in \mathcal{K}
\end{aligned}
$$

The above power minimization problem $\mathcal{P}_{\mathrm{PM}}$ can be efficiently solved either via SDR or via uplink power allocation algorithms by exploiting the uplink-downlink duality [6].

To apply the concept of CI exploitation to PM problems, firstly it should be noted that the SINR expression in (31) is no longer valid, since (i) the instantaneous symbol-level interference is related to not only the CSI but also the data symbols, and (ii) the interfering signals become constructive and contribute to the useful signal power. Following the discussion in Section II and recalling (11) and (14), the constructive PM (CPM) optimization for $\mathcal{M}$-PSK modulation based on the 'non-strict phase-rotation' metric can be expressed as [44]

$$
\begin{aligned}
\mathcal{P}_{\mathrm{CPM}}^{\mathrm{PSK}}: & \min _{\mathbf{x}}\|\mathbf{x}\|_{2}^{2} \\
\text { s.t. } & \mathrm{C} 1: \mathbf{h}_{k}^{\mathrm{T}} \mathbf{x}=\lambda_{k} s_{k}, \forall k \in \mathcal{K} \\
& \mathrm{C} 2:\left[\Re\left(\lambda_{k}\right)-\sqrt{\Gamma_{k} \sigma^{2}}\right] \tan \frac{\pi}{\mathcal{M}} \geq\left|\Im\left(\lambda_{k}\right)\right|, \forall k \in \mathcal{K}
\end{aligned}
$$

where $\Gamma_{k}$ is the SINR target for the $k$-th receiver. For convenience we have used $\mathbf{x}=\mathbf{W}$ s, since designing the precoding matrix $\mathbf{W}$ for CI-based SLP precoding is equivalent to directly designing the precoded signal vector $\mathbf{x}$. We further note that since the precoding matrix is symbol-dependent, the objective function also includes the data symbol vector $\mathbf{s}$, which guarantees that the transmit power is minimized on the symbol level, as discussed in Section I-C. Compared with traditional PM optimization $\mathcal{P}_{\mathrm{PM}}$ which is non-convex and has to resort to algorithms such as SDR, we observe that the constraints in $\mathcal{P}_{\text {CPM }}^{\text {PSK }}$ are linear and the constructed CPM is convex in nature, which can be efficiently solved via off-the-shelf optimization tools [87]. By following a similar procedure, the CPM formulation for QAM constellations can be constructed as

$$
\begin{array}{ll}
\mathcal{P}_{\mathrm{CPM}}^{\mathrm{QAM}}: & \min _{\mathbf{x}}\|\mathbf{x}\|_{2}^{2} \\
\text { s.t. } & \mathrm{C} 1: \mathbf{h}_{k}^{\mathrm{T}} \mathbf{x}=\mathbf{\Omega}_{k}^{\mathrm{T}} \mathbf{s}_{k}, \forall k \in \mathcal{K} \\
& \mathrm{C} 2: \alpha_{m}^{\mathcal{O}} \geq \sqrt{\Gamma_{k} \sigma^{2}}, \forall \alpha_{m}^{\mathcal{O}} \in \mathcal{O} \\
& \mathrm{C} 3: \alpha_{n}^{\mathcal{I}}=\sqrt{\Gamma_{k} \sigma^{2}}, \forall \alpha_{n}^{\mathcal{I}} \in \mathcal{I}
\end{array}
$$




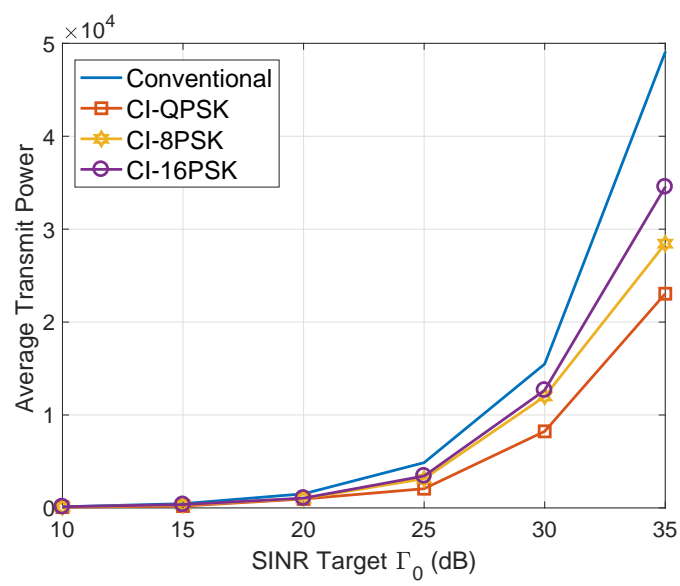

Fig. 6: Transmit power v.s. SINR threshold $\Gamma_{0}, \Gamma_{k}=\Gamma_{0}, \forall k$, $K=N_{t}=4, \sigma^{2}=1$

Similar to $\mathcal{P}_{\mathrm{CPM}}^{\mathrm{PSK}}$ for PSK constellations, the CPM problem for QAM constellations is also convex and can be readily solved.

To validate the significant transmit power savings that CI precoding exhibits, below in Fig. 6 we present a numerical example for CPM optimizations $\mathcal{P}_{\mathrm{CPM}}$ versus traditional PM formulation $\mathcal{P}_{\mathrm{PM}}$ that is solved via the SDR approach for several PSK constellations in a $4 \times 4$ MU-MISO system. Significant performance improvements can be observed for CIbased PM results over the data-independent PM result, with power savings up to $60 \%$ for QPSK, $40 \%$ for 8 PSK, and $20 \%$ for 16PSK, which reveals the superiority of interference exploitation in PM problems.

\section{SINR Balancing}

A typical SB optimization aims to improve the fairness in the wireless communication systems, by maximizing the minimum received SINR among the users subject to a total available transmit power budget at the BS [7], [8]. However, the SB problem is a non-convex optimization problem, which is in general difficult to obtain its optimal solution. To this end, a unified analytical framework is developed in [7], with which the optimal solution to the downlink SB optimization is shown to be equivalent to the optimum of a dual uplink problem. An efficient iterative algorithm that enjoys a fast convergence speed is also developed [7] to obtain this optimal solution. Meanwhile, [8] employs the conic optimization approaches to solve the downlink SB optimization, where an important 'inverse problem property' is revealed. Based on this property, it is shown in [8] that the SB optimization can be optimally solved by solving a set of PM problems via the bisectionsearch method. When a per-antenna power constraint instead of the sum-power constraint is considered, it is shown in [9] that the bisection-search framework is still effective to find the globally optimal solution by solving a set of dual per-antenna power balancing problems.

Based on the SINR expression in (31), a traditional SB optimization problem subject to a sum-power constraint can be formulated as [8]

$$
\begin{array}{ll}
\mathcal{P}_{\mathrm{SB}}: & \max _{\mathbf{w}_{k}} t \\
\text { s.t. } & \mathrm{C} 1: t \leq \frac{\left|\mathbf{h}_{k}^{\mathrm{T}} \mathbf{w}_{k}\right|^{2}}{\sum_{i \neq k}\left|\mathbf{h}_{k}^{\mathrm{T}} \mathbf{w}_{i}\right|^{2}+\sigma^{2}}, \forall k \in \mathcal{K} \\
& \mathrm{C} 2: \sum_{k=1}^{K}\left\|\mathbf{w}_{k}\right\|_{2}^{2} \leq P_{0}
\end{array}
$$

where $P_{0}$ is the transmit power allowance. Compared with PM optimizations, SB problems are more difficult to solve. Fortunately, the optimal solution to the SB problems can be obtained either through an iterative algorithm introduced in [7], or via a bisection-search method proposed in [8] based on the inverse problem property.

To extend CI exploitation to SB optimizations, we follow a similar procedure as done for PM optimizations and formulate a constructive SB (CSB) optimization problem for $\mathcal{M}$-PSK constellations, given by [58], [59]

$$
\begin{aligned}
\mathcal{P}_{\mathrm{CSB}}^{\mathrm{PSK}}: & \max _{\mathbf{x}} t \\
\text { s.t. } & \mathrm{C} 1: \mathbf{h}_{k}^{\mathrm{T}} \mathbf{x}=\lambda_{k} s_{k}, \forall k \in \mathcal{K} \\
& \mathrm{C} 2:\left[\Re\left(\lambda_{k}\right)-t\right] \tan \frac{\pi}{\mathcal{M}} \geq\left|\Im\left(\lambda_{k}\right)\right|, \forall k \in \mathcal{K} \\
& \mathrm{C} 3:\|\mathbf{x}\|_{2}^{2} \leq P_{0}
\end{aligned}
$$

Recalling Fig. 5, an interesting geometrical observation for $\mathcal{P}_{\mathrm{CSB}}^{\mathrm{PSK}}$ in (36) is that the optimization aims to maximize the distance between the 'constructive region' and the detection thresholds, such that the received signals that lie in the 'constructive region' are pushed as far away from the thresholds as possible. Moreover, an important observation for CPM and CSB optimizations is also revealed in 'Corollary 2' of [52], where it is shown that these two problems are inverse problems as well, which is similar to the case for conventional PM and SB optimizations. Based on this property, efficient algorithms based on the barrier method have been presented in [52], which are shown to be superior over the gradient descent algorithm that suffers from a low convergence speed.

The extension of CI-based SB problems to multi-level modulations is trivial, by incorporating the CI conditions for the corresponding multi-level constellations discussed in Section II-B in the formulated optimizations. For example, when QAM constellations are considered, the CSB optimization can be formulated as [60]

$$
\begin{aligned}
\mathcal{P}_{\mathrm{CSB}}^{\mathrm{QAM}}: & \max _{\mathbf{x}} t \\
\text { s.t. } & \mathrm{C} 1: \mathbf{h}_{k}^{\mathrm{T}} \mathbf{x}=\boldsymbol{\Omega}_{k}^{\mathrm{T}} \mathbf{s}_{k}, \forall k \in \mathcal{K} \\
& \mathrm{C} 2: t \leq \alpha_{m}^{\mathcal{O}}, \forall \alpha_{m}^{\mathcal{O}} \in \mathcal{O} \\
& \mathrm{C} 3: t=\alpha_{n}^{\mathcal{I}}, \forall \alpha_{n}^{\mathcal{I}} \in \mathcal{I} \\
& \mathrm{C} 4:\|\mathbf{x}\|_{2}^{2} \leq P_{0}
\end{aligned}
$$

The optimization problem formulation for APSK can be obtained in a similar way and is omitted for brevity.

Compared with traditional SB optimizations that are in general difficult to handle, the CI-based SB optimization $\mathcal{P}_{\mathrm{CSB}}^{\mathrm{PSK}}$ and $\mathcal{P}_{\mathrm{CSB}}^{\mathrm{QAM}}$ are both convex and can be readily solved via 


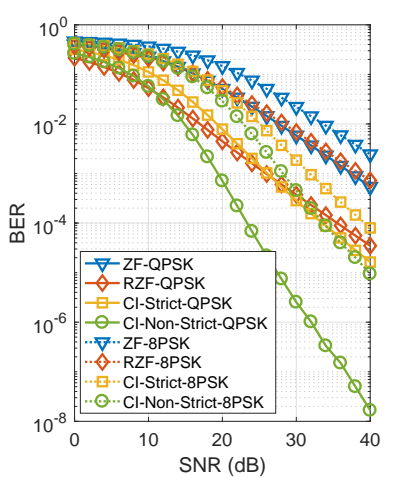

(a) PSK, Uncoded

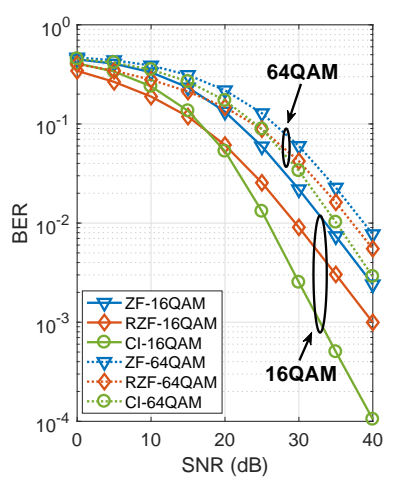

(b) QAM, Uncoded

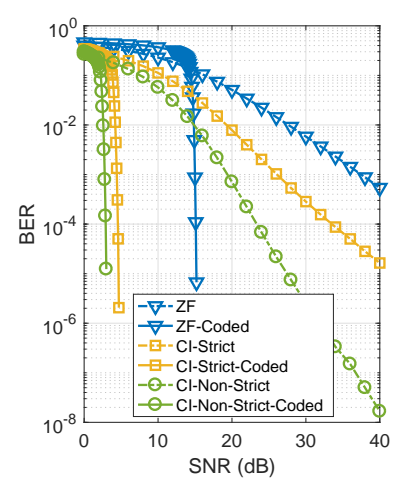

(c) PSK, Coded

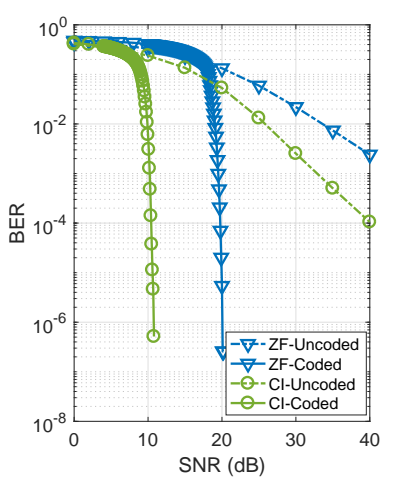

(d) QAM, Coded

Fig. 7: Uncoded and coded BER v.s. SNR, $K=N_{t}=12, P_{0}=1$, LDPC, code rate $1 / 4$

convex optimization tools to obtain their optimal solutions. Moreover, compared with traditional SB optimizations where an average transmit power is maintained, the symbol-level CSB optimization guarantees that the transmit power constraint is strictly met on a symbol-by-symbol basis, as observed in the power constraint which is on a symbol level. In addition, a closer look shows that $\mathcal{P}_{\mathrm{CVP}}$ that follows the symbol-scaling CI metric in Section III-A and $\mathcal{P}_{\mathrm{CSB}}^{\mathrm{PSK} / \mathrm{QAM}}$ in Section III-C are indeed equivalent problems and return the same precoded signals, based on the observation that the objective function $t$ in the CSB optimization is equal to the inverse of the power scaling factor $\beta_{\mathrm{CVP}}$, and therefore maximizing $t$ is equivalent to minimizing the power scaling factor.

To validate the superior performance of interference exploitation, we present a representative numerical BER result of CI precoding in Fig. 7 for a $12 \times 12$ MU-MISO system, where symbol-level ZF precoding and RZF precoding are employed as benchmarks for fairness of comparison. For PSK modulations, we depict the results for both the 'strict phase rotation' and 'non-strict phase rotation' CI metric, while we employ the 'symbol scaling' metric for QAM constellations. In Fig. 7a, we observe that the SNR gain for CI precoding based on 'non-strict phase rotation' is more than $10 \mathrm{~dB}$ for both QPSK and 8PSK, when compared with the RZF precoding. When the QAM constellation is employed instead, as shown in Fig. $7 \mathrm{~b}$, the SNR gain for CI precoding can still be up to $4.5 \mathrm{~dB}$ for $16 \mathrm{QAM}$ and $2.5 \mathrm{~dB}$ for $64 \mathrm{QAM}$ compared with RZF precoding. Additional numerical results presented in [60] show that the SNR gains can become more prominent when the system scales up. It is also observed that the performance gains extend to the case of coded BER results, when standard low-density parity-check (LDPC) codes are employed [88].

\section{Physical-Layer (PHY) Multicast Reformulation}

CI precoding has an interesting interpretation from the perspective of PHY multicasting [42], [44], [48] and [53]. PHY multicasting refers to the transmission where a common message is transmitted to all the receivers in the network, where no multi-user interference is observed since a single data stream is sent to all receivers [89]-[95]. The typical PM and SB problem for PHY multicasting is studied in [89], where both problems can be transformed into SDP forms and solved via SDR. To circumvent the drawback that SDR-based PHY multicasting is only effective when there are not too many users, as demonstrated in [90], a stochastic precoding is proposed in [90], which is irrespective of the number of users in the system, and the case with finite-alphabet inputs is further studied in [91]. Precoding for multiple co-channel multicast groups has been considered in [92]-[94], where a total transmit power constraint and a per-antenna power constraint is considered, respectively. The extension of PHY multicasting to a multi-cell network is investigated in [95].

From the interference point of view, as firstly revealed in [41], CI precoding considered in this paper resembles PHY multicasting precoding in a way that there exists no multi-user interference, where we note that CI precoding requires symbollevel operation, whereas multicasting does not. For PHY multicasting this is inherent because of single-stream transmission, while for CI precoding this is achieved by manipulating the interfering signals such that the resulting received signals lie in the constructive area and all the interference contributes to the useful signal power.

Mathematically, it has been shown in [44] and [53] that CPM optimization can be transformed and reformulated into a virtual PHY multicasting optimization. To be more specific, for the $\mathcal{P}_{\mathrm{CPM}}^{\mathrm{PSK}}$ problem shown in (33), by introducing a modified channel vector $\tilde{\mathbf{h}}_{k}=\frac{\mathbf{h}_{k}}{s_{k}}$ and with the introduced $\mathbf{x}=\mathbf{W s}$, $\mathcal{P}_{\text {CPM }}^{\text {PSK }}$ is equivalent to the following multicast problem [44]:

$$
\begin{aligned}
& \mathcal{P}_{\mathrm{CPM}}^{\mathrm{PHY}}: \min _{\mathbf{x}}\|\mathbf{x}\|_{2}^{2} \\
& \text { s.t. } \quad \mathrm{C} 1:\left[\Re\left(\tilde{\mathbf{h}}_{k}^{\mathrm{T}} \mathbf{x}\right)-\sqrt{\Gamma_{k} \sigma^{2}}\right] \tan \theta_{\text {th }} \geq\left|\Im\left(\tilde{\mathbf{h}}_{k}^{\mathrm{T}} \mathbf{x}\right)\right|, \forall k \in \mathcal{K}
\end{aligned}
$$

and it has been revealed that the optimal solution to $\mathcal{P}_{\mathrm{CPM}}^{\mathrm{PSK}}$ in (33) and $\mathcal{P}_{\mathrm{CPM}}^{\mathrm{PHY}}{ }^{\mathrm{M}}$ in (51) has the following connection [44]:

$$
\mathbf{w}_{k}^{*} s_{k}=\frac{\mathbf{x}^{*}}{K} \Rightarrow \mathbf{w}_{k}^{*}=\frac{\mathbf{x}^{*}}{K \cdot s_{k}} .
$$

Again, compared with traditional PHY multicasting [96] that is non-convex and needs to be solved via SDR methods, the virtual PHY multicasting formulation for CI precoding is 


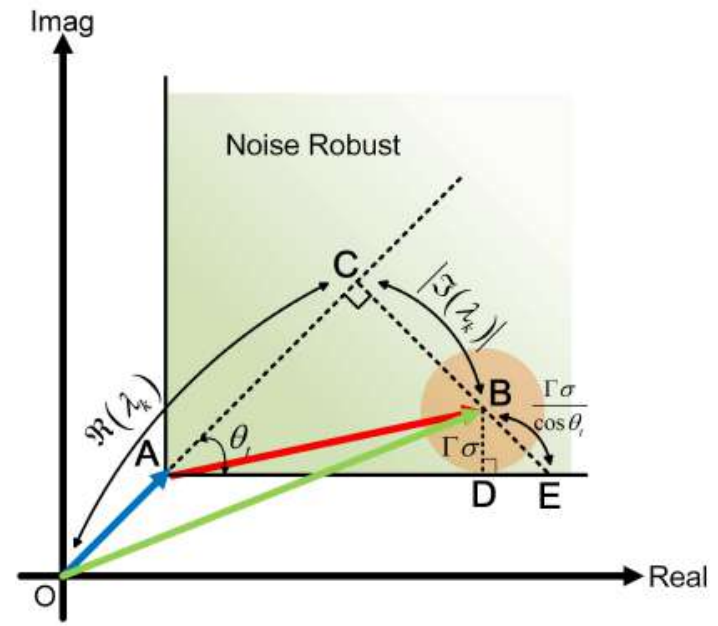

Fig. 8: Characterization for noise-robust CI condition

convex and can be readily solved. In addition to the above multicasting reformulation for the CI metric employed in [44], some similar multicast reformulations can also be found in [42] and [48] for the CI metric considered in the corresponding works.

\section{E. Symbol Error Probability (SEP) Optimization}

It should be noted that many CI-based precoding works [39]-[48] have constructed their CI formulations based on the noiseless received signal $\mathbf{h}_{k}^{\mathrm{T}} \mathbf{W} \mathbf{s}$, as observed in (11) where the effect of noise is not taken into account, which may lead to sub-optimal solutions. To fill in this gap, [51], [52], [66] and [68] consider the noise-robust CI condition, where given a pre-defined noise variance, the received symbol still falls into the correct detection region of the constellation. As shown in Fig. 8, for a given noiseless received signal $\overrightarrow{O B}$, the noise uncertainty region (denoted by the brown zone) needs to be incorporated into the precoding design, such that the received signal including noise still satisfies the SNR target $\Gamma_{k}$. Specifically, [52] aims to design the precoding matrix such that when the noise is located in the noise uncertainty region, the received signal including noise still falls into the constructive region of the constellations, which can be correctly decoded. Based on the geometry in Fig. 8, the updated noise-robust CI condition for a $\mathcal{M}$-PSK constellation can be mathematically expressed as [52]

$$
\Re\left(\lambda_{k}\right) \tan \frac{\pi}{\mathcal{M}}-\frac{\Gamma_{k} \sigma}{\cos \frac{\pi}{\mathcal{M}}} \geq\left|\Im\left(\lambda_{k}\right)\right|, \forall k \in \mathcal{K} .
$$

By incorporating the noise-robust CI condition (40) into the precoding design, the corresponding CPM and CSB can be formulated as [52]

$$
\begin{array}{cl}
\mathcal{P}_{\mathrm{NR}-\mathrm{CPM}}^{\mathrm{PSK}}: & \min _{\mathbf{x}}\|\mathbf{x}\|_{2}^{2} \\
\text { s.t. } & \mathrm{C} 1: \mathbf{h}_{k}^{\mathrm{T}} \mathbf{x}=\lambda_{k} s_{k}, \forall k \in \mathcal{K} \\
& \mathrm{C} 2: \Re\left(\lambda_{k}\right) \tan \frac{\pi}{\mathcal{M}}-\frac{\Gamma_{k} \sigma}{\cos \frac{\pi}{\mathcal{M}}} \geq\left|\Im\left(\lambda_{k}\right)\right|, \forall k \in \mathcal{K}
\end{array}
$$

and

$$
\begin{array}{cl}
\mathcal{P}_{\mathrm{NR}-\mathrm{CSB}}^{\mathrm{PSK}}: & \max _{\mathbf{x}} t \\
\text { s.t. } & \mathrm{C} 1: \mathbf{h}_{k}^{\mathrm{T}} \mathbf{x}=\lambda_{k} s_{k}, \forall k \in \mathcal{K} \\
& \mathrm{C} 2: \Re\left(\lambda_{k}\right) \tan \frac{\pi}{\mathcal{M}}-\frac{t \sigma}{\cos \frac{\pi}{\mathcal{M}}} \geq\left|\Im\left(\lambda_{k}\right)\right|, \quad \forall k \in \mathcal{K} \\
& \mathrm{C} 3:\|\mathbf{x}\|_{2}^{2} \leq P_{0}
\end{array}
$$

respectively. Moreover, a closer observation shows that $\mathcal{P}_{\mathrm{CSB}}^{\mathrm{PSK}}$ in (36) and $\mathcal{P}_{\mathrm{NR}-\mathrm{CSB}}^{\mathrm{PSK}}$ are indeed equivalent problems and return the same solution, based on the fact that

$$
\begin{aligned}
& \Re\left(\lambda_{k}\right) \tan \frac{\pi}{\mathcal{M}}-\frac{t \sigma}{\cos \frac{\pi}{\mathcal{M}}} \geq\left|\Im\left(\lambda_{k}\right)\right| \\
\Rightarrow & {\left[\Re\left(\lambda_{k}\right)-\frac{t \sigma}{\sin \frac{\pi}{\mathcal{M}}}\right] \tan \frac{\pi}{\mathcal{M}} \geq\left|\Im\left(\lambda_{k}\right)\right| }
\end{aligned}
$$

and maximizing $\frac{t \sigma}{\sin \frac{\pi}{\mathcal{M}}}$ is equivalent to maximizing $t$ itself.

[52] also presents an alternative SEP-based CI precoding, where the detection-region based CI constraint in (40) is replaced by a probabilistic constraint. By defining the SEP as the probability when the received signals including noise falls out of the decision region of a specific constellation point, where incorrect detection occurs, the SEP-based CI condition for a $\mathcal{M}$-PSK constellation can be derived as [52]

$$
\Re\left(\lambda_{k}\right) \tan \frac{\pi}{\mathcal{M}}-\frac{\operatorname{erf}^{-1}(1-2 p) \sigma}{\cos \frac{\pi}{\mathcal{M}}} \geq\left|\Im\left(\lambda_{k}\right)\right|, \forall k \in \mathcal{K},
$$

where $\operatorname{erf}^{-1}(\cdot)$ is the Gaussian error function. $p$ is the target SEP for a PM optimization, while $p$ becomes the objective function when a SB optimization is considered. By incorporating (44) into the problem formulation, the corresponding SEP-based CPM and CSB optimizations can be constructed accordingly, which is omitted for brevity. The connection between CI precoding based on the noise-robust design in (40) and CI precoding based on SEP in (44) is also revealed in [52].

\section{F. Closed-Form Iterative Precoding}

Compared with traditional block-level precoding approaches that are optimized when the channel changes, it should be noted that CI precoding has to perform optimizations on a symbol level, and the resulting computational complexity becomes an important issue for all CI-based precoding approaches. Therefore, low-complexity solutions and efficient algorithms have become an indispensable part for the realization of CI precoding. In the literature, several efficient algorithms have already been proposed for CI precoding, as detailed in [42], [44], [52], [56].

Thanks to the simple structures of the objective function as well as the linear constraints for CI-based PM and SB optimizations in Section III-B and Section III-C, recently it has been shown in [58]-[60] that there exist an optimal precoding structure for CI precoding. Based on the introduction of $\mathbf{x}=\mathbf{W s}$ and the observation that how the power is distributed among $\mathbf{w}_{i} s_{i}$ does not affect the solution, it is therefore safe to assume that each $\mathbf{w}_{i} s_{i}$ is identical, and the power constraint 
for the CSB optimization in (36) and (37) can be further transformed into [58]

$$
\|\mathbf{x}\|_{2}^{2} \leq P_{0} \Rightarrow \sum_{i=1}^{K} s_{i}^{*} \mathbf{w}_{i}^{\mathrm{H}} \mathbf{w}_{i} s_{i} \leq \frac{P_{0}}{K} .
$$

Based on this equivalent power constraint, by analyzing the optimization problem $\mathcal{P}_{\mathrm{CSB}}^{\mathrm{PSK}}$ via Karush-Kuhn-Tucker (KKT) conditions and further formulating the dual problem, it is shown that the optimal precoded signal $\mathrm{x}$ for PSK modulation can be expressed as [58]

$$
\mathbf{x}=\mathbf{H}^{\mathrm{H}}\left(\mathbf{H H}^{\mathrm{H}}\right)^{-1} \operatorname{diag}\left(\sqrt{\frac{P_{0}}{\mathbf{u}^{\mathrm{T}} \mathbf{S} \mathbf{T}^{-1} \mathbf{S}^{\mathrm{T}} \mathbf{u}}} \mathbf{U T}^{-1} \mathbf{S}^{\mathrm{T}} \mathbf{u}\right) \mathbf{s} .
$$

We refer the interested readers to [58] for a detailed derivation and the expressions for $\mathbf{S}, \mathbf{T}$, and $\mathbf{U}$. Based on this closedform expression, it is observed in [58] that CI precoding operates on a symbol level, as both $\mathbf{T}$ and $\mathbf{u}$ are dependent on the data symbol s. Another interesting interpretation based on (46) is that CI precoding can be viewed as the combination of ZF precoding with a pre-scaling operation that is adaptive to the data symbols. In fact, it has been shown in [58] that CI precoding can be regarded as a generalization of $\mathrm{ZF}$ precoding, and ZF precoding is a special case of CI precoding when the scaling factors $\lambda_{k}$ in (11) are all identical. $\mathbf{u} \in \mathcal{R}^{2 K \times 1}$ in (46) needs to be optimized, which can be obtained via the following optimization [58]:

$$
\begin{array}{cl}
\mathcal{P}_{\mathbf{u}}^{\text {PSK }}: & \min _{\mathbf{u}} \mathbf{u}^{\mathrm{T}}\left(\mathbf{S T}^{-1} \mathbf{S}^{\mathrm{T}}\right) \mathbf{u} \\
\text { s.t. } & \mathrm{C} 1: \mathbf{1}^{\mathrm{T}} \mathbf{u}=1 \\
& \mathrm{C} 2: u_{i} \geq 0, \forall i \in\{1,2, \cdots, 2 K\}
\end{array}
$$

which is a standard QP optimization over a simplex.

When a QAM constellation is considered instead, the optimal precoded signal can be obtained in a similar way, which is given by [60]

$$
\mathbf{x}=\mathbf{H}^{\mathrm{H}}\left(\mathbf{H H}^{\mathrm{H}}\right)^{-1} \mathbf{U d i a g}\left(\sqrt{\frac{P_{0}}{\mathbf{u}^{\mathrm{T}} \mathbf{V}^{-1} \mathbf{u}}} \mathbf{F}^{-1} \mathbf{V}^{-1} \mathbf{u}\right) \mathbf{s}_{\mathbf{E}},
$$

and we refer the interested readers to [60] for detailed derivations. The corresponding dual vector $\mathbf{u}$ can also be obtained via a QP optimization, while in the case of QAM modulation the QP is no longer optimized over a simplex, since only $u_{m}$ that corresponds to $\alpha_{m}^{\mathcal{O}} \in \mathcal{O}$ needs to be constrained as non-negative. By following the steps as in [58]-[60], the optimal precoding structure for CPM optimizations and the corresponding QP formulations can be derived in a similar way. Meanwhile in the literature, an exact closed-form but sub-optimal solution has also been derived for CPM problems based on the DPCIR metric in [57].

We further note the complexity reduction with the derived optimal precoding structure. Compared with the original CPM and CSB optimizations that belong to second-order cone programming (SOCP), the formulated optimizations for $\mathbf{u}$ are $\mathrm{QP}$ problems, which can be more efficiently solved using the interior-point methods, as widely acknowledged in the literature [97]-[99]. Moreover, for both PSK and QAM constellations, an efficient iterative algorithm is also developed to solve the corresponding QP optimization, as detailed in [58] and [60]. This proposed algorithm allows a closed-form solution within each of its iteration, and obtains the optimal QP solution within only a few iterations. Another important feature for the iterative closed-form algorithm proposed in [58] and [60] is that it returns a feasible precoding matrix after each iteration, which is a great advantage over other efficient algorithms based on gradient descent method [42], [44], [56] or barrier method [52]. To be more specific, the iterative closed-form algorithm starts with ZF precoding, and evolves to the optimal CI precoding with the iteration number increasing, which offers a flexible performance-complexity tradeoff compared with other algorithms and makes it most appealing in practical systems, where performance has to be compromised for complexity reduction.

In addition, it should be highlighted that the above optimal precoding structure results and the corresponding QP formulations are based on the conventional case where $K \leq N_{t}$ and $\left(\mathbf{H H}^{\mathrm{H}}\right)^{-1}$ is applicable. As already mentioned in Section I-C, CI precoding also supports the case of $K>N_{t}$, and the corresponding precoding structure for PSK and QAM modulation has been derived in [59] and [60], respectively. By exploiting the singular value decomposition (SVD), the expression for $\mathbf{W}$ in the case of $K>N_{t}$ immediately follows (46) and (48) by substituting $\left(\mathbf{H H}^{\mathrm{H}}\right)^{-1}$ with the pseudoinverse $\left(\mathbf{H H}^{\mathrm{H}}\right)^{+}$[100], while it should be noted that the expression for $\mathbf{T}$ in (46) and $\mathbf{V}$ in (48) is different in the case of $K>N_{t}$, which then leads to a different QP objective function.

\section{G. Robust CI Precoding}

The above studies and results on CI precoding have been derived based on the assumption that perfect CSI is known at the transmitter side, which is however not achievable since perfect CSI is not available in a practical wireless communication system. Therefore, it is important to consider a more realistic scenario and design robust CI-based precoding, when CSI is not perfect at the transmitter.

In the case of imperfect CSI, an additive error model is usually considered and the actual channel can be modeled as [101]

$$
\mathbf{h}_{k}=\hat{\mathbf{h}}_{k}+\mathbf{e}_{k},
$$

where $\hat{\mathbf{h}}_{k}$ denotes the estimated CSI known at the BS, and $\mathbf{e}_{k}$ represents the CSI uncertainty. The additive channel estimation errors can be modeled into two different forms, dependent on the duplex mode of the communication system [101]. In the time-division duplex (TDD) mode, the CSI can be directly estimated at the BS using the uplink-downlink reciprocity and is subject to estimation errors. In this case, the entry of $\mathbf{e}_{k}$ can be modeled as a zero-mean Gaussian random variable, whose variance is inversely proportional to the transmit SNR. With this statistical CSI error model, the robust design is usually designed by optimizing the outage performance [102], [103]. On the other hand, when the frequency-division duplex (FDD) mode is considered, the CSI error $\mathbf{e}_{k}$ is usually modeled as 
norm bounded to characterize the quantization errors incurred by limited feedback [101]. In this case, the channel uncertainty can be considered as bounded by a spherical region $\mathcal{V}_{k}=$ $\left\{\mathbf{e}_{k} \mid\left\|\mathbf{e}_{k}\right\|_{2}^{2} \leq \delta_{k}^{2}\right\}$, and the robust precoding algorithms are usually designed based on the worst-case received SINR [102], [104]-[106]. The robust PM optimization in the case of normbounded CSI errors can usually be formulated as [105]

$$
\begin{aligned}
\mathcal{P}_{\mathrm{R}-\mathrm{PM}}: & \min _{\mathbf{w}_{k}} \sum_{k=1}^{K}\left\|\mathbf{w}_{k}\right\|_{2}^{2} \\
\text { s.t. } & \mathrm{C} 1: \gamma_{k} \geq \Gamma_{k}, \forall \mathbf{e}_{k} \in \mathcal{V}_{k}, \forall k \in \mathcal{K} \\
& \mathrm{C} 2: \mathbf{h}_{k}=\hat{\mathbf{h}}_{k}+\mathbf{e}_{k}, \forall k \in \mathcal{K}
\end{aligned}
$$

This conventional robust PM problem $\mathcal{P}_{\text {R-PM }}$ can be transformed into a SDP form and solved via the SDR approach [107].

To date, several works have considered the robust design for CI-based precoding [44], [108], [109]. In [44], the robust version of CI precoding for both $\mathrm{PM}$ and $\mathrm{SB}$ optimization against norm-bounded CSI errors is studied, where the worstcase robust design for CI precoding is formulated based on the multicast formulation. By expanding the complex representation of the considered problem into its real equivalence, the robust version of the CI condition for a generic PSK modulation is derived. Mathematically, this leads to the following CI-based robust precoding design [44]:

$$
\begin{aligned}
\mathcal{P}_{\mathrm{R}-\mathrm{CPM}}^{\mathrm{PSK}}: & \min _{\mathbf{t}_{1}, \mathbf{t}_{2}}\left\|\mathbf{t}_{1}\right\|_{2}^{2} \\
\text { s.t. } \quad \mathrm{C} 1: & \left(\hat{\mathbf{h}}_{k}^{\mathrm{E}}\right)^{\mathrm{T}} \mathbf{t}_{1}-\left(\hat{\mathbf{h}}_{k}^{\mathrm{E}}\right)^{\mathrm{T}} \mathbf{t}_{2} \tan \theta_{\mathrm{th}}+\delta_{k}\left\|\mathbf{t}_{1}-\mathbf{t}_{2} \tan \theta_{\mathrm{th}}\right\|_{2} \\
& +\sqrt{\Gamma_{k} \sigma^{2}} \tan \theta_{\mathrm{th}} \leq 0, \forall k \in \mathcal{K} \\
\mathrm{C} 2: & -\left(\hat{\mathbf{h}}_{k}^{\mathrm{E}}\right)^{\mathrm{T}} \mathbf{t}_{1}-\left(\hat{\mathbf{h}}_{k}^{\mathrm{E}}\right)^{\mathrm{T}} \mathbf{t}_{2} \tan \theta_{\mathrm{th}}+\delta_{k}\left\|\mathbf{t}_{1}-\mathbf{t}_{2} \tan \theta_{\mathrm{th}}\right\|_{2} \\
& +\sqrt{\Gamma_{k} \sigma^{2}} \tan \theta_{\mathrm{th}} \leq 0, \forall k \in \mathcal{K} \\
\mathrm{C} 3: & \mathbf{t}_{1}=\mathbf{\Pi t}_{2}
\end{aligned}
$$

where

$$
\mathbf{t}=\mathbf{W} \mathbf{s}, \mathbf{t}_{1}=\left[\mathbf{t}_{\Im}^{\mathrm{T}}, \mathbf{t}_{\Re}^{\mathrm{T}}\right]^{\mathrm{T}}, \mathbf{t}_{2}=\left[\mathbf{t}_{\Re}^{\mathrm{T}},-\mathbf{t}_{\Im}^{\mathrm{T}}\right]^{\mathrm{T}},
$$

and

$$
\hat{\mathbf{h}}_{k}^{\mathrm{E}}=\left[\left(\hat{\mathbf{h}}_{k}^{\Re}\right)^{\mathrm{T}},\left(\hat{\mathbf{h}}_{k}^{\Im}\right)^{\mathrm{T}}\right]^{\mathrm{T}}, \boldsymbol{\Pi}=\left[\begin{array}{cc}
\mathbf{0}_{K} & \mathbf{I}_{K} \\
-\mathbf{I}_{K} & \mathbf{0}_{K}
\end{array}\right] .
$$

$\mathcal{P}_{\mathrm{R}-\mathrm{CPM}}^{\mathrm{PSK}}$ is a standard SOCP formulation and can be solved efficiently [44]. It is shown that compared with traditional PM and SB optimizations [6] and [7], the transmit power savings for CI-based robust design can be as large as $3 \mathrm{~dB}$ when the error bound is $\delta_{k}^{2}=0.0005$, which can become more prominent when the value of the error bound increases.

In addition, when only quantized CSI is available at the transmitter, [108] divides the interference into predictable interference that can be manipulated to be constructive, and unpredictable interference caused by the quantization in the CSI. Based on the quantized CSI error model, the proposed method in [108] aims to enhance the useful signal power by controlling the predictable interference, while minimizing the effect of uncertainty from unpredictable interference. An upper bound of the unpredictable interference is firstly derived, based on which the formulated optimization problem is transformed into a convex one and an iterative algorithm based on the gradient descent method is further introduced.

Alternative robust CPM optimizations have recently been proposed in [109], where both the statistical and normbounded CSI error model are considered. When a normbounded CSI error model is assumed, the worst-case CI constraint is firstly formulated and a robust CPM optimization is proposed, which is similar to [44]. On the other hand, when the statistical CSI errors are assumed, the robust approach is designed based on the probabilistic CI constraints, which is equivalent to designing the precoding matrix such that the probability of violating the CI constraint is below a predefined threshold. By applying a decorrelation transformation and employing a lower bound instead, a linear inequality constraint is derived in [44], based on which the robust CPM optimization against statistical CSI errors can be formulated as a convex optimization problem and solved efficiently.

\section{H. Summary, Insights and Lessons Learnt}

In this section, we have reviewed CI-based SLP techniques in the downlink transmission of a MU-MISO system, which are shown to be more prominent than traditional closed-form and optimization-based precoding designs in terms of error rate performance (both with and without channel coding) and transmit power savings, as verified by the numerical results. In the following, we conclude this section by highlighting the insights of CI-based SLP techniques.

In terms of the CI metric, while currently the "phaserotation' CI metric seems to be more popular in the literature, it is a fact that the 'symbol-scaling' metric is indeed proposed earlier than the 'phase-rotation' metric, which first appeared in the context of VP precoding in [39]. In the current literature, the 'phase-rotation' metric is preferred when PSK modulations are considered, while the 'symbol-scaling' metric has to be adopted when QAM modulations are studied. In addition, the derived noise-robust CI condition (40) in Section III-E is shown to be equivalent to the traditional 'phase-rotation' $\mathrm{CI}$ metric, since optimizing the scaled version of a variable is equivalent to optimizing the variable itself.

In terms of the problem formulation, compared with traditional PM and SB problems that are non-convex, it is shown that CI-based PM and SB problems are convex and can be optimally solved via off-the-shelf convex optimization tools. This is another advantage for CI precoding over traditional optimization-based precoding approaches that are generally more complicated and difficult to be employed in practical wireless communication systems. In addition, the PHY multicast reformulation provides an interesting interpretation for CI precoding. Conceptually, CI precoding is similar to PHY multicast in a way that there exists no multi-user interference. Mathematically, the virtual PHY multicast reformulation further reveals that the optimal precoding vector for one data symbol is a phase-rotated version of the optimal precoding vector for another data symbol, as shown in (39) when PSK signaling is considered. This is further validated by the derived optimal precoding structure in Section III-F. 
The derivation of the optimal closed-form precoding structure for CI-based techniques has been a breakthrough in the field of interference exploitation, which is motivated by the PHY multicast reformulation in Section III-D. For the first time in the literature, it reveals that CI precoding can be seen as a combination of traditional $\mathrm{ZF}$ precoding and a symbol-level scaling for the data symbol vector. Thanks to its closed-form expression, the derived precoding structure further motivates the analytical performance analysis for CI precoding, as recently studied in [110] and [111] for PSK signaling, where the expressions for the achievable rate, the total sum rate upper bound and the SER of CI transmissions are derived.

\section{Applications of CI-BAsed Precoding}

In the previous section, we have shown the CI formulation of conventional PM and SB optimizations, and numerically demonstrated significant performance improvements for CI precoding in terms of error rate and transmit power savings in Fig. 6 and Fig. 7. In this section, we discuss how CI precoding can be adapted to other wireless scenarios and the potential gains of exploiting interference in these scenarios.

\section{A. Cognitive Radio $(C R)$}

Compared with traditional fixed spectrum allocation strategy, CR that enables dynamic spectrum access is an effective way to increase the radio resource utilization and spectral efficiency, which has been extensively studied [10], [112][114]. Dependent on the priority of accessing the spectrum, the users are divided into primary users (PUs) and secondary users (SUs) in underlay CR networks, where PUs have the highest priority for the spectrum resources without being aware of the existence of the SUs in the network, while SUs can only access the network under the premise that their interference to PUs is below a certain threshold [114]. Accordingly, a fundamental challenge for $\mathrm{CR}$ networks is to enable the opportunistic spectrum access for SUs while guaranteeing PUs' quality-ofservice $(\mathrm{QoS})$ requirements, when the CSI of both PUs and SUs is available at the BS.

The tradeoff between throughput maximization and interference minimization for SUs is studied in [115] from an information-theoretic perspective, where the optimal transmission scheme that achieves the capacity of the secondary transmission as well as some sub-optimal algorithms is presented. In addition, precoding designs for CR networks are studied in [116]-[119] for both perfect CSI and imperfect CSI, where a precoding scheme termed MSLNR, which is a combination of the optimal minimum-mean-squared-error (MMSE) receiver and the signal-to-leakage-plus-noise ratio (SLNR) transmitter, is proposed in [116]. A joint downlink precoding and power control optimization is considered in [117] to maximize the weighted sum rate. The formulated non-convex problem is tackled by exploiting the non-negative matrix theory, where a convex approximation as well as a single-input multiple-output (SIMO)-MISO duality is further established. A robust precoding design for a single-SU CR network is considered in [118] in the presence of imperfect
CSI, where the service probability of the SU is maximized through an iterative algorithm. Specifically, a closed-form solution can be obtained in the case of only one PU. A robust linear precoding design is further proposed in [119] for an underlay CR network with multiple PUs, where the formulated optimization is transformed into a convex-concave problem based on the uplink-downlink duality, and an iterative SDP-based algorithm is presented. Typically, a max-min fair problem in the $\mathrm{CR}$ Z-channel that aims to maximize the minimum SINR subject to average interference power to the PUs and total transmit power constraint at the secondary BS can be formulated as [120]

$$
\begin{array}{cl}
\mathcal{P}_{\mathrm{CR}-\mathrm{SB}}: & \max _{\mathbf{w}_{k}} \min _{k} \gamma_{k} \\
\text { s.t. } & \mathrm{C} 1: \sum_{i=1}^{K}\left|\mathbf{g}_{l}^{\mathrm{T}} \mathbf{w}_{i}\right|^{2} \leq \varepsilon_{l}, \quad \forall l \in \mathcal{L} \\
& \mathrm{C} 2: \sum_{k=1}^{K}\left\|\mathbf{w}_{k}\right\|_{2}^{2} \leq P_{0}
\end{array}
$$

where $\mathcal{L}=\{1,2, \cdots, L\}, \mathrm{g}_{l}$ denotes the channel vector between the secondary $\mathrm{BS}$ and the $l$-th $\mathrm{PU}$, and $\varepsilon_{l}$ is the maximum allowed interference power. $\mathrm{C} 1$ therefore guarantees that the interference for the PUs from the secondary BS is below the required threshold. The formulated problem $\mathcal{P}_{\mathrm{CR}-\mathrm{SB}}$ can be solved via the bisection-search method and sequential QP [121]-[123]. Additional robust precoding designs for CR networks against imperfect CSI can be found in [124]-[127], and CR has also been combined with relay in [128]-[130].

CI-based precoding has shown to be effective in CR networks [120], [131]-[136]. Early works include [131] and [132] based on selective CI precoding and correlation-rotation CI precoding, where a parallel transmission system aided with a cognitive relay is considered in [131] while an overlay CR network is investigated in [132]. The corresponding performance advantages of $\mathrm{CI}$ precoding over ZF precoding have been shown numerically in terms of SER, for both considered scenarios. More recently, the optimization-based CI precoding method proposed in [44] has been applied to the CR Zchannel in [120], [133] and [135]. Compared with [131] and [132] that adopt the 'strict phase-rotation' CI metric with computationally-efficient closed-form CI precoders, the optimization-based approach [135] that adopts the same CI metric is expected to achieve an improved performance, which is however more computationally expensive than [131] and [132] since it needs to solve an optimization problem on a symbol level. [120] and [133] adopt the more advanced 'non-strict phase-rotation' CI metric over [135] and therefore lead to additional transmit power savings compared with [135]. Particularly, [120] derives the condition under which the formulated probabilistic precoding design can be transformed into a convex deterministic optimization, based on which a simple approximation method that allows a convenient SOCP formulation is further introduced for additional reduction in the computational costs. The hybrid analog-digital precoding structure is further applied to CR scenarios in [134], which exhibits significant total power savings at the BS compared with the traditional fully-digital architectures. 
Mathematically, the optimization problem that aims to maximize the minimum SINR subject to the total transmit power constraint and the interference power constraint can be formulated as

$$
\begin{aligned}
\mathcal{P}_{\text {CI-CR-SB }}^{\mathrm{PSK}}: & \max _{\mathbf{x}} t \\
\text { s.t. } & \mathrm{C} 1: \mathbf{h}_{k}^{\mathrm{T}} \mathbf{x}=\lambda_{k} s_{k}, \forall k \in \mathcal{K} \\
& \mathrm{C} 2:\left[\Re\left(\lambda_{k}\right)-t\right] \tan \frac{\pi}{\mathcal{M}} \geq\left|\Im\left(\lambda_{k}\right)\right|, \forall k \in \mathcal{K} \\
\mathrm{C} 3: & \left|\mathbf{g}_{l}^{\mathrm{T}} \mathbf{x}\right|^{2} \leq \varepsilon_{l}, \forall k \in \mathcal{L} \\
\mathrm{C} 4: & \|\mathbf{x}\|_{2}^{2} \leq P_{0}
\end{aligned}
$$

Compared with traditional interference-reduction max-min fair optimizations, an SNR gain of $10 \mathrm{~dB}$ can be observed for the CI-based precoding for the underlay $\mathrm{CR}$ Z-channel scenario in terms of SER, when the SBS is equipped with 10 transmit antennas with a total number of 8 SUs and 2 PUs in the network, as illustrated in [120].

\section{B. Simultaneous Wireless Information and Power Transfer (SWIPT)}

Energy harvesting (EH) and wireless power transfer for wireless communication networks have become a new paradigm that allows user equipments (UEs) to prolong the battery life [11], [12]. In wireless communications, the emitted RF signals carry both the information and energy at the same time, and therefore SWIPT techniques that enable the simultaneous transmission of information symbols and energy to the UEs have become particularly appealing [137], [138]. In [138], three types of SWIPT scenarios are introduced: (i) separate UEs where each individual UE is acting as either an EH receiver or an information decoding (ID) receiver; (ii) time-switching UEs where the UEs are acting as EH receivers within some symbol durations while as ID receivers within other symbol durations; and (iii) power-splitting UEs where UEs divide the power of the received signals into two parts, one part for ID and the other part for EH.

Some precoding designs for MIMO SWIPT systems have been studied in [139]-[142]. [139] and [140] consider the separate-UE SWIPT scenario, where in [139] the ZF precoding method is employed for MIMO SWIPT systems, and it is shown that the harvested energy obtained by the EH receivers can be increased at the cost of an SINR loss of the ID receivers. [140] designs the precoding approaches which aim to maximize the harvested energy for $\mathrm{EH}$ receivers while guaranteeing the SINR target of the IDs, and the globally optimal solutions are obtained via the SDR method. A powersplitting SWIPT scenario is studied for a MISO multicasting system in [141] and for a unicast system in [142], where the joint optimization on the precoding vector and the receive power splitting ratio is investigated. When power-splitting UEs are assumed, as the most common considered case, the typical PM formulation for a unicast SWIPT system can be expressed as $[142]$

$$
\begin{aligned}
\mathcal{P}_{\text {SWIPT }}: & \min _{\mathbf{w}_{k}, \rho_{k}} \sum_{k=1}^{K}\left\|\mathbf{w}_{k}\right\|_{2}^{2} \\
\text { s.t. } & \mathrm{C} 1: \frac{\left|\mathbf{h}_{k}^{\mathrm{T}} \mathbf{w}_{k}\right|^{2}}{\sum_{i \neq k}\left|\mathbf{h}_{k}^{\mathrm{T}} \mathbf{w}_{i}\right|^{2}+\sigma^{2}+\frac{\sigma_{\mathrm{C}}^{2}}{\rho_{k}}} \geq \Gamma_{k}, \forall k \in \mathcal{K} \\
& \mathrm{C} 2:\left(1-\rho_{k}\right)\left(\sum_{i=1}^{K}\left|\mathbf{h}_{k}^{\mathrm{T}} \mathbf{w}_{i}\right|^{2}+\sigma^{2}\right) \geq E_{k}, \forall k \in \mathcal{K} \\
& \mathrm{C} 3: 0 \leq \rho_{k} \leq 1, \forall k \in \mathcal{K}
\end{aligned}
$$

where $\sigma_{\mathrm{C}}^{2}$ is the additional noise power introduced in the RF to baseband conversion, $E_{k}$ is the harvested energy threshold for UE $k, \rho_{k}$ represents the fraction of power for ID, and $\left(1-\rho_{k}\right)$ is the fraction of power for $\mathrm{EH}$. $\mathrm{C} 1$ and $\mathrm{C} 2$ are the downlink received SINR and harvested energy requirements, respectively. This formulated PM problem, as well as the PM formulation for multicast case in [141], can be efficiently solved via the SDR method, which is shown to be tight in both scenarios. In addition to the above studies, the joint information and energy precoding methods for SWIPT are also investigated in MIMO interference channels in [143]-[146], and SWIPT techniques have been combined with PHY security in [147]-[150] by considering the broadcast nature of wireless communication systems. In [151] and [152], the hybrid analogdigital structure has been applied to SWIPT scenarios, which demonstrates noticeable total power savings at the BSs.

The concept of CI exploitation has been applied to powersplitting SWIPT systems in [153]-[155]. Compared with traditional SWIPT systems that regard multi-user interference as harmful in the ID process, CI-based SWIPT precoding methods take advantages of the CI, which is inherent in the downlink transmission, as an additional power source for both useful information and wireless energy. A joint downlink precoding and receive power splitting optimization is formulated as a PM problem, subject to the QoS target for the ID receivers and EH thresholds for the EH receivers, given by [155]

$\mathcal{P}_{\text {CI-SWIPT }}^{\text {PSK }} \min _{\mathbf{x}, \rho_{k}}\|\mathbf{x}\|_{2}^{2}$

s.t. $\mathrm{C} 1: \mathbf{h}_{k}^{\mathrm{T}} \mathbf{x}=\lambda_{k} s_{k}, \forall k \in \mathcal{K}$

$\mathrm{C} 2:\left[\Re\left(\lambda_{k}\right)-\sqrt{\Gamma_{k}\left(\sigma^{2}+\frac{\sigma_{\mathrm{C}}^{2}}{\rho_{k}}\right)}\right] \tan \theta_{\mathrm{th}} \geq\left|\Im\left(\lambda_{k}\right)\right|, \forall k \in \mathcal{K}$

$\mathrm{C} 3:\left|\mathbf{h}_{k}^{\mathrm{T}} \mathbf{x}\right| \geq \sqrt{\frac{E_{k}}{1-\rho_{k}}}, \forall k \in \mathcal{K}$

$\mathrm{C} 4: 0 \leq \rho_{k} \leq 1, \forall k \in \mathcal{K}$

Compared with PM problems for interference-reduction MIMO SWIPT systems where the PM problems can be transformed and readily solved via SDR approaches, CI-based PM problems for MIMO SWIPT systems are more difficult to handle due to the non-convex EH constraints. This is opposed to many other application scenarios of CI techniques where usually the adoption of the $\mathrm{CI}$ condition leads to simplification of the optimization problems. In [153], the CI-based non- 
convex problem is handled by using difference of convex optimization and the successive linear approximation method, which returns a sub-optimal solution. [154] and [155] further improve the schemes proposed in [153], where SOCP-based and SDP-based algorithms with polynomial complexity are further introduced, which provide the upper and lower bounds to the optimal solution, respectively. By further conducting the asymptotic analysis, the optimality of these algorithms are established when the modulation order and SINR target go to infinity. By allowing a SLP design for MIMO SWIPT systems, the CI-based approach exhibits a transmit power saving as large as $8.6 \mathrm{dBw}$ when $E_{k}=-30 \mathrm{dBm}$ and $9.2 \mathrm{dBw}$ when $E_{k}=-10 \mathrm{dBm}$, compared with traditional precoding designs based on SDR.

\section{Physical-Layer (PHY) Security}

Compared with wired communications, a wireless communication system is naturally more susceptible and vulnerable to security threats due to its broadcast nature. Traditionally, the security issues are handled at the network layer by keybased cryptographic techniques. Nevertheless, PHY security approaches, which artificially add structured redundancy in the transmit signals in the physical layer such that the legitimate users can correctly decode the confidential information while the eavesdroppers (Eves) cannot, have drawn increasing research attention in the information-theoretic society in recent years [13]-[16]. By employing PHY transmission schemes that are specifically designed for security using multiple antennas, PHY security techniques can improve the information security and act as an additional security layer on top of the traditional cryptographic approaches.

One possible approach for realizing PHY security is through downlink precoding, which is able to direct the signals carrying confidential information to the legitimate users while minimizing the power leakage to the Eves, as studied in [156][158], where the secrecy rate maximization is discussed when the BS has either perfect or imperfect CSI of the Eves. Another promising approach for PHY security is to send generated artificial noise (AN) signals to interfere the Eves deliberately, as firstly proposed in [159] and further investigated in [160][162]. The design for the AN-aided PHY security techniques is largely dependent on whether the BS has the CSI of the Eves. When no CSI of the Eves is available at the BS, a popular design is to generate an isotropic AN that is uniformly spread in the null space of the legitimate channel [159]. By doing so, the communication for the legitimate users is not affected by the AN while the reception of the Eves is degraded. If the knowledge of the Eves' CSI is known perfectly or partially at the $\mathrm{BS}$, this information can further be exploited to generate spatially selective AN that is much more effectively than the isotropic AN, as shown in [160]-[162]. Specifically, when the BS aims to transmit confidential messages to a legitimate user in the presence of $K$ Eves with full CSI available, the ANaided secure PM optimization subject to QoS constraints can be formulated as [160]

$$
\begin{array}{ll}
\mathcal{P}_{\text {PHY-S }}: & \min _{\mathbf{w}, \mathbf{z}}\|\mathbf{w}\|_{2}^{2}+\|\mathbf{z}\|_{2}^{2} \\
\text { s.t. } & \mathrm{C} 1: \frac{\left|\mathbf{h}_{\mathrm{L}}^{\mathrm{T}} \mathbf{w}\right|^{2}}{\left|\mathbf{h}_{\mathrm{L}}^{\mathrm{T}} \mathbf{z}\right|^{2}+\sigma^{2}} \geq \Gamma_{\mathrm{L}} \\
& \mathrm{C} 2: \frac{\left|\mathbf{h}_{\mathrm{E}, k}^{\mathrm{T}} \mathbf{w}\right|^{2}}{\left|\mathbf{h}_{\mathrm{E}, k}^{\mathrm{T}} \mathbf{z}\right|^{2}+\sigma^{2}} \leq \Gamma_{\mathrm{E}, k}, \forall k \in \mathcal{K}
\end{array}
$$

where $\mathbf{h}_{\mathrm{L}}$ is the channel between the legitimate user and the $\mathrm{BS}$, and $\mathbf{h}_{\mathrm{E}, k}$ is the channel between the $k$-th eavesdropper and the BS. C1 guarantees that the received SINR for the legitimate user meets a pre-defined threshold for correct detection, while C2 ensures that the Eves cannot correctly decode the confidential information. The above optimization $\mathcal{P}_{\text {PHY-S }}$ can readily be solved by the SDR method as shown in [160] and [162]. Several other representative works on PHY security can be found in [163]-[165] for two-way relay networks and [166] for transmit antenna selection (AS).

It has been shown in the literature that CI-based precoding can be applied to PHY security techniques for additional performance improvements [167]-[171]. [167] and [168] focus on the practical scenario where the legitimate transmitter does not have the knowledge of its channel to the eavesdropper, based on which security-enhancing techniques through SLP are proposed for different transmitter and eavesdropper antenna configurations. Compared with [167] and [168], [169] considers the CI-based secure precoding design for both the ideal case where the channel knowledge of the legitimate users and the eavesdroppers is available and the practical case where the legitimate transmitter does not have access to the eavesdroppers' CSI. It is demonstrated that when the CSI of the Eves is not known to the BS, the AN design can be shifted from an isotropic null space based method to a CIbased method for transmit power savings, which is similar to the idea of conventional interference exploitation. On the other hand, in the case where partial/full CSI is available, it allows a more advanced CI-based approach where the AN signals are designed to be constructive to the legitimate users and destructive to the Eves at the same time, which further reduces the required transmit power at the BS while impeding the signal detection at the Eves. More recently, [170] reexamines the destructive region for PHY security scenarios in [169] and introduces the 'complete destructive region', based on which several new secure precoding designs are proposed for a single-eavesdropper scenario with full, statistical and no Eve's CSI respectively, which are shown to be superior to the secure precoding designs in [169].

When PSK modulations are employed, the optimization problem for CI-based precoding design in PHY security can 
mathematically be formulated as [169]

$$
\begin{aligned}
\mathcal{P}_{\text {CI-PHY-S }}^{\mathrm{PSK}}: \min _{\mathbf{x}_{\mathrm{L}}, \mathbf{z}}\left\|\mathbf{x}_{\mathrm{L}}+\mathbf{z}\right\|_{2}^{2} \\
\text { s.t. } \mathrm{C} 1: \mathbf{h}_{\mathrm{L}}^{\mathrm{T}}\left(\mathbf{x}_{\mathrm{L}}+\mathbf{z}\right)=\lambda_{\mathrm{L}} s_{\mathrm{L}} \\
\text { C2 }: \mathbf{h}_{\mathrm{E}, k}^{\mathrm{T}}\left(\mathbf{x}_{\mathrm{L}}+\mathbf{z}\right)=\lambda_{\mathrm{E}, k} s_{\mathrm{L}}, \forall k \in \mathcal{K} \\
\text { C3 : }\left[\Re\left(\lambda_{\mathrm{L}}\right)-\sqrt{\Gamma_{\mathrm{L}} \sigma^{2}}\right] \tan \theta_{\mathrm{th}} \geq\left|\Im\left(\lambda_{\mathrm{L}}\right)\right| \\
\text { C4 : }\left[\Re\left(\lambda_{\mathrm{E}, k}\right)-\sqrt{\Gamma_{\mathrm{E}, k} \sigma^{2}}\right] \tan \theta_{\mathrm{th}} \leq \Im\left(\lambda_{\mathrm{E}, k}\right), \forall k \in \mathcal{K} \\
\text { C5 }:\left[\Re\left(\lambda_{\mathrm{E}, k}\right)-\sqrt{\Gamma_{\mathrm{E}, k} \sigma^{2}}\right] \tan \theta_{\mathrm{th}} \geq-\Im\left(\lambda_{\mathrm{E}, k}\right), \forall k \in \mathcal{K}
\end{aligned}
$$

where C3 guarantees CI for the legitimate user, while $\mathrm{C} 4$ and C5 ensure that the AN signals are destructive to the Eves. $\mathcal{P}_{\text {CI-PHY-S }}^{\text {PSK }}$ is a standard SOCP problem and can be efficiently solved with interior-point based solvers. Similar benefits for CI-based design over interference-reduction designs are also observed when PHY security is combined with SWIPT, as studied in [171].

\section{Full-Duplex (FD) Communications}

Traditional wireless communication systems work either in FDD or TDD mode, with both being the half-duplex (HD) model. To meet the high demand for spectral efficiency and QoS requirement of the future wireless systems, FD communications, which allow simultaneous transmission and reception, have been considered as one of the solutions [17][19]. While FD transmission can theoretically double the spectral efficiency of the HD systems, the self interference from the transmit antennas to the receive antennas of the FD transceivers can severely affect the quality of communication in practical scenarios. Thanks to the recent major breakthroughs for practical FD systems which allow a self interference cancellation of up to $100 \mathrm{~dB}$ with the use of additional hardware, as illustrated in [172], [173], FD communications have received increasing research attention in recent years [174]-[180].

In [174], a pricing-based precoding method specifically tailored for the suppression of self interference is proposed, which guarantees the linearity of the receiver and achieves a spectral efficiency that is nearly 1.8 times of a HD system. In [175], precoding approaches for a sum-rate maximization in FD systems are designed based on the sequential convex programming, and a joint consideration of forward precoding and self interference cancellation is further studied in [176]. A downlink precoding and uplink multi-user combining problem for sum-rate maximization in FD systems is investigated in [177]. The formulated non-convex optimization is handled by exploiting the uplink-downlink duality, and a minorizationmaximization (MM) algorithm is proposed. The practical deployment of a multi-user MIMO system with a FD BS and HD UEs is considered in [178], where a joint optimization in precoding designs and uplink/downlink user selection is proposed. For a typical multi-objective optimization problem that aims to jointly minimize the total downlink and uplink transmit power for the considered FD system, the optimization problem can be formulated as [179], [180]

$$
\begin{aligned}
& \mathcal{P}_{\mathrm{FD}}: \min _{\mathbf{w}_{k}, P_{j}} \max _{a=1,2}\left\{\eta_{a}\left(Q_{a}^{*}-Q_{a}\left(\mathbf{w}_{k}, P_{j}\right)\right)\right\} \\
& \text { s.t. } \quad \mathrm{C} 1: \frac{\left|\mathbf{h}_{k}^{\mathrm{T}} \mathbf{w}_{k}\right|^{2}}{\sum_{i \neq k}\left|\mathbf{h}_{k}^{\mathrm{T}} \mathbf{w}_{i}\right|^{2}+\sigma^{2}} \geq \Gamma_{k}^{\mathrm{DL}}, \forall k \in \mathcal{K} \\
& \mathrm{C} 2: \frac{P_{j}\left|\mathbf{u}_{j}^{\mathrm{T}} \mathbf{g}_{j}\right|^{2}}{\sum_{n \neq j} P_{n}\left|\mathbf{u}_{j}^{\mathrm{T}} \mathbf{g}_{n}\right|^{2}+\sum_{k=1}^{K}\left|\mathbf{u}_{j}^{\mathrm{T}} \mathbf{H}_{\mathrm{SI}} \mathbf{w}_{k}\right|^{2}+\sigma^{2}\left\|\mathbf{u}_{j}\right\|_{2}^{2}} \geq \Gamma_{j}^{\mathrm{UL}} \\
& \forall j \in \mathcal{J}
\end{aligned}
$$$$
\text { C3 : } P_{j} \geq 0, \forall j \in \mathcal{J}
$$

where $\mathrm{C} 1$ and $\mathrm{C} 2$ are to guarantee the downlink and uplink SINR requirement, respectively. We refer the interested readers to [179] and [180] for a detailed explanation on the notations in $\mathcal{P}_{\mathrm{FD}}$, where it is also shown that the formulated precoding design for FD communications can be solved via the SDR approach. Additional studies on FD communications include applications to PHY security in [181]-[183], and FD relays in [184]-[187].

CI exploitation can also be applied to FD communications for additional performance improvements, as recently studied in [188]-[190] for both PSK and QAM constellations. The CIbased PM problem in a multi-user FD system is considered in [188] and [189], where a multi-objective optimization via the weighted Chebycheff method is employed to study the tradeoff between the two desirable design objectives, which are the total downlink transmit power at the BS and the total uplink power from the UEs. When PSK constellations are considered, the corresponding CI-based optimization for FD communications can be formulated as [189]

$$
\begin{aligned}
& \mathcal{P}_{\mathrm{CI}-\mathrm{FD}}^{\mathrm{PSK}}: \min _{\mathbf{x}, P_{j}, t} t \\
& \text { s.t. } \quad \mathrm{C} 1: \mathbf{h}_{k}^{\mathrm{T}} \mathbf{x}=\lambda_{k} s_{k}, \forall k \in \mathcal{K} \\
& \mathrm{C} 2:\left[\Re\left(\lambda_{k}\right)-\sqrt{\Gamma_{k}^{\mathrm{DL}} \sigma^{2}}\right] \tan \theta_{\mathrm{th}} \geq\left|\Im\left(\lambda_{k}\right)\right|, \forall k \in \mathcal{K} \\
& \mathrm{C} 3: \frac{P_{j}\left|\mathbf{u}_{j}^{\mathrm{T}} \mathbf{g}_{j}\right|^{2}}{\sum_{n \neq j} P_{n}\left|\mathbf{u}_{j}^{\mathrm{T}} \mathbf{g}_{n}\right|^{2}+\left|\mathbf{u}_{j}^{\mathrm{T}} \mathbf{H}_{\mathrm{SI}} \mathbf{x}\right|^{2}+\sigma^{2}\left\|\mathbf{u}_{j}\right\|_{2}^{2}} \geq \Gamma_{j}^{\mathrm{UL}} \\
& \mathrm{C} 4: \eta_{a}\left(Q_{a}^{*}-Q_{a}\left(\mathbf{x}, P_{j}, t\right)\right) \leq t, \forall a \in\{1,2\} \quad \forall j \in \mathcal{J} \\
& \mathrm{C} 5: P_{j} \geq 0, \forall j \in \mathcal{J}
\end{aligned}
$$

where $\mathrm{C} 2$ is the CI-adapted SINR requirement for the downlink transmission. $\mathcal{P}_{\mathrm{CI}-\mathrm{FD}}^{\mathrm{PSK}}$ is shown to be a convex problem and can be readily solved. Numerical results have shown a $2 \mathrm{dBw}$ downlink transmit power saving and a $7 \mathrm{dBw}$ uplink transmit power saving by exploiting the $\mathrm{CI}$ in a system where FD BS is equipped with 8 antennas with a total number of 6 downlink users and 3 uplink users, which also leads to the substantial reduction in the self interference. Importantly, the multi-objective framework allows the flexible tradeoff between the uplink power savings and the downlink power savings, which further leads to an improvement in the overall energy 
efficiency. In addition, CI-based FD communications have also been considered in the presence of imperfect CSI for energy harvesting scenarios in [190], where a robust design for the joint minimization of the uplink and downlink transmit power and maximization of the harvested energy is studied subject to channel estimation errors. It is shown by numerical results that the gain in the harvested energy can be as large as $5 \mathrm{dBm}$ with an increase in the downlink transmit power.

\section{E. Multi-Cell Distributed Antennas (DAs)}

A DA system is a wireless communication architecture where multiple geographically DAs are connected to a central processing unit through distributed remote radio heads (RRHs) [191], [192], where in the literature it is also referred to as 'BS cooperation', 'network MIMO' or 'multi-cell processing', etc. While consuming an increased backhaul requirement compared with traditional centralized multi-antenna BSs, DA systems intuitively have the advantage of greatly reducing the path loss of the communication links, thanks to the reduced distance between the transmitting antennas and receiving UEs. Therefore, the DA system is a promising architecture to reduce the required transmit power of the BS for a fixed channel quality and leads to a more uniform coverage inside the cell [191], [192]. Due to these benefits, DA systems have received considerable research attention, which includes the studies on spectral efficiency [193]-[196], energy efficiency [197] and their tradeoffs [198], power allocation schemes for energy efficiency maximization in [199]-[201], precoding designs in [201]-[204], and AS strategies in [205].

More recently, several DA systems have also been proposed in the massive MIMO regime, for example the "cell-free massive MIMO' in [206]-[210]. Cell-free massive MIMO systems employ a large number of access points (APs) equipped with single antenna or a few antennas geographically distributed over a wide area, which exhibits significant throughput improvements compared with small-cell deployments [206]. In a cell-free massive MIMO system, the concept of 'cell' does not exist, since the entire area is covered by distributed APs that cooperate phase-coherently via a backhaul network to the central processing unit, which names this DA system [206]. One key feature of cell-free massive MIMO systems is that, only local signal processing at each RRH is sufficient without the need for centralized processing, when the MRT precoding is employed [206]-[208]. In addition to the simple MRT precoding, [209] and [210] investigate the cell-free massive MIMO systems when ZF precoding is employed, where power control algorithms are presented for SB optimization in [209] and for energy efficiency maximization in [210]. In addition to cell-free massive MIMO architectures, there is another promising DA system termed 'fog massive MIMO' that has recently been proposed in [211] and [212], which exploits a small number of RRHs with each RRH deploying a large-scale antenna array.

The concept of CI precoding has been extended to DA systems in [213], where the PHY security is enhanced by CI precoding for a user-centric DA system. A joint CI-based secure precoding and a DA selection method is proposed for transmit PM problem, in which the AN signals are designed to be constructive to the UEs while destructive to the Eves. The formulated problems are discussed for the case when only imperfect CSI is known as well as the case when the CSI of the Eves is completely unknown. As a step further, CI precoding has also been extended to a multi-cell scenario in [214] based on both deterministic optimization and probabilistic optimization, where several coordination schemes are proposed for downlink transmission. A fully-coordinated strategy is firstly considered, where both the intra-cell interference and the intercell interference are exploited for performance improvements based on the CI formulation, by sharing the information of both the channel and data symbols among the BSs. A partially-coordinated scheme is also introduced for coordination overhead reduction, where the intra-cell interference is manipulated to be constructive while the inter-cell interference is suppressed by only sharing the CSI among the BSs. In a numerical example where 3 BSs cooperate with 3 users in each cell, [214] shows a $4 \mathrm{dBm}$ power saving gain based on the deterministic optimization and a $6 \mathrm{dBm}$ power saving gain based on the probabilistic optimization.

\section{F. Spatio-Temporal CI: Faster-than-Nyquist Signaling}

Faster-than-Nyquist (FTN) signaling [215]-[218] is a signal processing scheme allowing a notable improvement of the spectral efficiency of wireless communication systems. The key idea of FTN signaling is a reduction of the time spacing between two adjacent pulses (the symbol period) below the one satisfying the Nyquist condition. In other words, in FTN signaling the data rate is increased by accelerating the transmitted pulses in the temporal dimension (time packing), thus introducing controlled inter-symbol interference (ISI) which needs to be handled. The main problem of FTN signaling is the need to cope with the introduced ISI, which in turn results in complex receivers relying on trellis decoders as well as ad-hoc equalization schemes, whose computational costs are often prohibitive in practical applications. In [219]-[221], a novel transmission technique has been proposed, which merges the aggressive frequency reuse relying on precoding, in particular SLP, with FTN signaling. In a generic MUMISO system, these works extend the concept of SLP at the transmitter side in order to tackle not only the interference in the spatial dimension (the multi-user interference), but also the interference in the temporal dimension (the ISI intentionally introduced through FTN signaling). Such an extension allows FTN signaling in a MU-MISO framework and, at the same time, solves the problem of complex FTN receivers, as the ISI is completely handled at the transmitter. This transmission technique is referred to as spatio-temporal CI, as it enhances the $\mathrm{CI}$ both in the temporal and in the spatial dimensions, thus gleaning benefits from both the domains.

The application of SLP in the context of FTN signaling relies on a new system model, which takes into account the temporal variation of the transmitted streams at each antenna by modeling the pulse shaping filters. Considering a MUMISO system with $N_{\mathrm{T}}$ transmit antennas and $K$ single-antenna user terminals, the main idea is to split each data stream in 
temporal blocks of $S$ symbols. The data symbols for the different users, for a given block, can be represented in a matrix $\mathbf{S}=\left[\mathbf{s}_{1} \ldots \mathbf{s}_{K}\right]^{\mathrm{T}} \in \mathcal{C}^{K \times S}$, while the precoded symbol streams can be aggregated in a matrix $\mathbf{D}=\left[\mathbf{d}_{1} \ldots \mathbf{d}_{N_{\mathrm{T}}}\right]^{\mathrm{T}} \in \mathcal{C}^{N_{\mathrm{T}} \times S}$. Denoting $T$ as the symbol period and $\mu$ as the oversampling factor, the pulse-shaped transmitted waveform for the generic $n$-th antenna can be represented through its discrete samples, spaced by $t_{s}=\frac{T}{\mu}$, given by [219]

$x_{n}[m]=\sum_{j=1}^{S} d_{n}[j] \alpha\left[(m-1) t_{s}-(j-1) T\right], m=1, \ldots, \mu S$,

where $\alpha(t)$ represents the considered unit energy pulse and $d_{n}[j]$ is the $j$-th element of the symbol vector $\mathbf{d}_{n}$. The output (oversampled) signals from all the antennas can be aggregated in a matrix $\mathbf{X}=\left[\mathbf{x}_{1} \ldots \mathbf{x}_{N_{\mathrm{T}}}\right]^{\mathrm{T}} \in \mathcal{C}^{N_{\mathrm{T}} \times \mu S}$. With this definition, a compact way to represent the pulse shaping operation is $\mathbf{X}=\mathbf{D A}_{\mathrm{TX}}$, with $\mathbf{A}_{\mathrm{TX}} \in \mathcal{R}^{S \times \mu S}$ being a block Toeplitz matrix including the filter taps.

By aggregating the received symbols at the $K$ users in a matrix $\mathbf{Y} \in \mathcal{C}^{K \times S}$, the spatio-temporal communication model can be written as [219], [221]

$$
\mathbf{Y}=\mathbf{H X} \mathbf{A}_{\mathrm{RX}}+\tilde{\mathbf{Z}} \mathbf{A}_{\mathrm{RX}}=\mathbf{H D A}+\mathbf{Z}
$$

with $\tilde{\mathbf{Z}}$ being the noise in the oversampled domain, $\mathbf{A}_{\mathrm{RX}} \in$ $\mathcal{R}^{\mu S \times S}$ modeling the matched filtering and downsampling operation performed at each receiver, and $\mathbf{A}=\mathbf{A}_{\mathrm{TX}} \mathbf{A}_{\mathrm{RX}} \in$ $\mathcal{R}^{S \times S}$ representing the combination of the filters at the transmitter and at the receiver. The overall spatio-temporal system model, accounting for the ISI through $\mathbf{A}$ and the multi-user interference through $\mathbf{H}$, is represented in the block scheme of Fig. 9. By vectorizing the introduced signal matrices over the temporal dimension, the spatio-temporal communication model can be expressed as [219]

$$
\mathbf{y}=\left(\mathbf{H} \otimes \mathbf{A}^{\mathrm{T}}\right) \mathbf{d}+\mathbf{z}=\mathbf{G} \mathbf{d}+\mathbf{z},
$$

which is formally similar to the spatial model of (3) used in the traditional SLP literature. The matrix $\mathbf{G}=\mathbf{H} \otimes \mathbf{A}^{\mathrm{T}} \in$ $\mathcal{C}^{K S \times N_{\mathrm{T}} S}$ is an equivalent representation of the channel matrix in this spatio-temporal model.

The above spatio-temporal communication model has been used in [219] to formulate a CI-PM problem with QoS constraints, which is mathematically equivalent to the PM problem of [222]. The related problem is convex and can be solved resorting to $\mathrm{CVX}$. Moreover, a sequential approach has been proposed in [219] and [221], where the residual

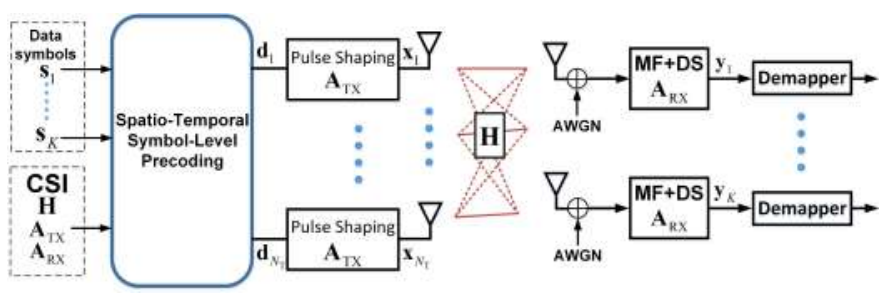

Fig. 9: Block diagram of the SLP approach based on spatiotemporal CI
ISI amongst the subsequent temporal blocks is also tackled. Numerical results have shown that SLP schemes based on FTN can outperform the Nyquist-based counterparts both in terms of energy efficiency and achievable rate, with performance gains up to $25 \%$.

\section{G. Summary, Insights and Lessons Learnt}

In this section, we have discussed the adaptation of CI precoding to a number of wireless scenarios. By transforming interference into useful signal power, CI-based techniques also exhibit noticeable performance improvements in these scenarios, as summarized below.

It is quite straightforward to extend the concept of interference exploitation to CR scenarios. This is because from a mathematical point of view, $\mathrm{CR}$ scenarios only introduce an additional constraint to the optimization problem, which constrains the interference power to the PUs below a certain threshold, when a typical CR-Z channel is considered. Thus, the CI approaches can be readily extended to CR scenarios by incorporating the CI condition into the problem formulation, as shown in (55).

In terms of the application in SWIPT scenarios, CI-based techniques however lead to more complicated problem formulations compared with traditional interference-reduction approaches, which is opposed to various other wireless scenarios where incorporating the CI condition usually leads to simplified problem formulation. This is because of the nonconvex harvested energy constraint, which is handled by SDP reformulation in traditional approaches. However, the CI condition cannot be transformed into a SDP form, which makes the CI-based SWIPT problems difficult to handle, and thus [153]-[155] have adopted the successive linear approximation method to obtain a feasible solution.

The benefits of applying CI-based techniques to PHY security are two folds, as shown in [169] and [170]. On one hand, the multi-user interference can be made constructive to the transmission of the legitimate users to further benefit their detection performance, which is similar to the application of $\mathrm{CI}$ in the downlink transmissions of traditional multi-antenna systems. On the other hand, when the CSI of the eavesdroppers is available at the BS, CI-based designs in PHY security can be designed such that the AN signals are further destructive to the eavesdroppers at the same time, which further degrades the detection performance for the potential eavesdroppers.

Similar to the case of PHY security, we can observe the benefits of CI-based techniques from two perspectives when applied to FD communication systems. On one hand, CI-based FD precoding approaches lead to downlink transmit power savings at the BS, which is similar in traditional CI-based PM problems. Meanwhile, due to the joint optimization in both the uplink transmission and downlink transmission, interference exploitation also allows uplink transmit power savings for the first time in the CI literature. Both of the above benefits further lead to the improvement in terms of energy efficiency.

Finally, the application of CI-based SLP approaches in FTN signaling is quite insightful to interference exploitation. First of all, by incorporating the spatio-temporal communication 


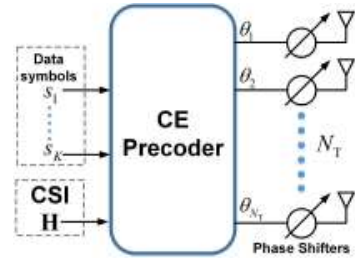

(a) Constant-envelope precoding

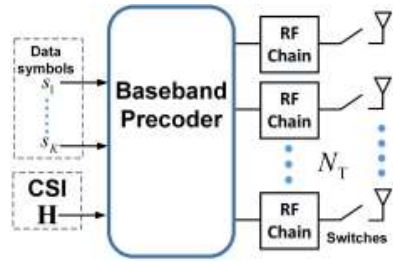

(b) Antenna selection

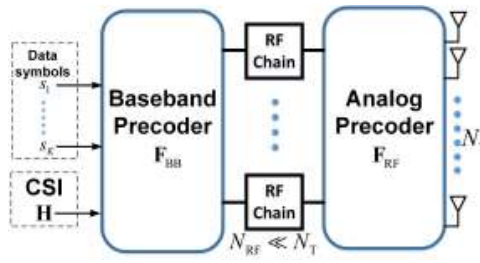

(c) Hybrid precoding

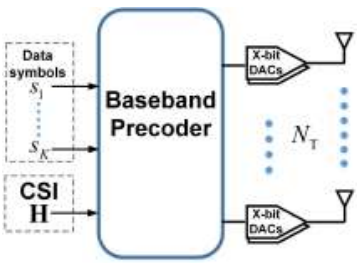

(d) Low-resolution DACs

Fig. 10: A variety of hardware-efficient BS architecture

model, the CI-based techniques can be directly applied to the scenario of FTN signaling, which leads to noticeable performance gains in terms of energy efficiency and achievable sum rate compare with traditional FTN-based approaches. Moreover, the benefits that CI-based techniques are able to deliver in FTN signaling further motivate the use of CI-based approaches in a broader range of interference scenarios, such as ISI and adjacent-channel interference (ACI).

\section{Symbol-Level PreCoding For Hardware EFFICIENCY}

In this section, we further extend the concept of CI exploitation to hardware-constrained large-scale antenna systems for hardware efficiency, which includes constant-envelope precoding (CEP), AS, hybrid analog-digital (AD) precoding, lowresolution digital-to-analog converters (DACs), non linearities, and RF-domain SLP. Aimed at improving both the cost efficiency and energy efficiency of the BS, these schemes attempt to reduce the complexity of the BS architecture and the number of some hardware components, as shown in Fig. 10 and Fig. 12, respectively.

\section{A. Constant-Envelope Precoding (CEP)}

In recent years, the use of a large-scale antenna array at the transmitter has been shown to offer remarkable benefits compared with a small-scale MIMO system [223], [224]. Unlike traditional small-scale MIMO systems that employ highly linear and power-inefficient radio frequency (RF) amplifiers, the practical implementation of large-scale MIMO systems requires the RF amplifiers to be power efficient, otherwise the consequent power consumption of the BS would be prohibitively high. Unfortunately, power-efficient RF amplifiers usually experience poor linearity characteristics and therefore further require the input signals to have a low PAPR. Accordingly, CE transmission, which enforces each antenna element to emit $\mathrm{CE}$ signals and allows the use of the most power-efficient and cheapest power amplifiers, as shown in Fig. 10a, has become an active research direction [225]-[229], which holds great potential for the practical implementation of large-scale antenna systems.

In [225], a single-user single-stream CE transmission is considered, where it is shown that in this case the noiseless received signal region is doughnut shaped. Based on this observation, a near-optimal capacity-achieving input distribution is derived. It is further revealed in [226] that the inner radius of the doughnut-shaped region has a closed-form expression.
A joint optimization of CEP and antenna subset selection is proposed in [227] from a geometric perspective for a singleuser MISO system. CEP has been further extended to a multiuser case in [228], where the CEP problem is formulated as a non-linear least-squares (NLS) optimization to minimize the multi-user interference, given by [228]

$$
\begin{gathered}
\mathcal{P}_{\mathrm{CEP}}: \quad \min _{\boldsymbol{\theta}} \sum_{k=1}^{K}\left|\sum_{n=1}^{N_{\mathrm{T}}} h_{k, n} e^{j \theta_{n}}-\sqrt{E_{k}} s_{k}\right|^{2} \\
\text { s.t. } \quad \mathrm{C} 1:\left|\theta_{n}\right| \leq \pi, \forall n \in\left\{1,2, \cdots, N_{\mathrm{T}}\right\}
\end{gathered}
$$

where $\sqrt{E_{k}}$ is the magnitude of user $k$ 's data symbol $s_{k}$, $h_{k, n}$ is the $n$-th entry of $\mathbf{h}_{k}$, and $\boldsymbol{\theta}=\left[\theta_{1}, \theta_{2}, \cdots, \theta_{N_{\mathrm{T}}}\right]^{\mathrm{T}}$. An iterative algorithm is presented in [228] to efficiently obtain the phases of the CE signals. Building upon this, a cross-entropy optimization (CEO) method is introduced in [229] for $\mathcal{P}_{\mathrm{CEP}}$, which achieves an improved performance over [228]. Some other works include CEP for frequency-selective channels in [230], a joint transceiver design for CEP in a point-to-point (P2P) MIMO system in [231], CEP for MISO multicasting in [232], [233], and CEP with quantized phases in [234].

A closer look at the above studies reveals that CEP has to operate on a symbol level, since the phases of the CE signals are dependent on the information of the data symbols. From this point of view, CI-based precoding that also requires a symbol-level operation can be a perfect match with CEP. The concept of CI exploitation has been applied to CEP firstly in [235] and [236], where instead of minimizing the multi-user interference, constructive CEP aims to maximize the CI effect subject to transmitting $\mathrm{CE}$ signals at each antenna element, which can be formulated as [236]

$$
\begin{array}{cl}
\mathcal{P}_{\mathrm{C}-\mathrm{CEP}}^{\mathrm{PSK}}: & \max _{\mathbf{x}} t \\
\text { s.t. } & \mathrm{C} 1: \mathbf{h}_{k}^{\mathrm{T}} \mathbf{x}=\lambda_{k} s_{k}, \forall k \in \mathcal{K} \\
& \mathrm{C} 2:\left[\Re\left(\lambda_{k}\right)-t\right] \tan \theta_{\text {th }} \geq\left|\Im\left(\lambda_{k}\right)\right|, \forall k \in \mathcal{K} \\
& \mathrm{C} 3:\left|x_{n}\right|=\sqrt{\frac{P_{0}}{N_{\mathrm{T}}}}, \forall n \in\left\{1,2, \cdots, N_{\mathrm{T}}\right\}
\end{array}
$$

where $\mathbf{x}=\left[x_{1}, x_{2}, \cdots, x_{N_{\mathrm{T}}}\right]^{\mathrm{T}}$. $\mathcal{P}_{\mathrm{C}-\mathrm{CEP}}^{\mathrm{PSK}}$ is generally non-convex because of the non-convex constraint $\mathrm{C} 3$. To address this non-convex problem, a CEO-based and a two-step CVXbased approach are proposed in [235] and [236] for $\mathcal{P}_{\mathrm{C}-\mathrm{CEP}}^{\mathrm{PSK}}$ respectively, both of which are shown to achieve significant performance improvements over the traditional CEP methods in [228] and [229] based on interference minimization. While not explicitly mentioned, it should be noted that the CEP approach for MISO multicasting proposed in [232], which 
maximizes the minimum scaling effect among the users, is also based on the concept of interference exploitation by employing the symbol-scaling CI metric. Recently, [237] studies the CEP problem from the perspective of Riemannian manifold. By approximating the real representation of the original problem and mapping it onto a Riemannian manifold, an efficient Riemannian conjugate gradient algorithm is proposed, and additional performance improvements in terms of SER are observed compared with the CEP methods in both [235] and [236]. The above studies in [235]-[237] show that the concept of interference exploitation can be readily extended to CEP problems and significant performance benefits are observed. When quantized phases are considered, CI-based CEP problems are studied in [238] for both PSK and QAM signaling.

Additionally, the SLP techniques proposed for hardwareefficient architectures in [239] are also candidates for CE transmission. While the proposed architectures include gain control at the transmitter, as contrary to CEP, it is shown that if the gain is kept constant regardless of the channel realization or the transmitted symbols, the derived algorithm that is based on the coordinate descent method is shown to be an efficient solution for CE transmission, which is able to reduce multiuser interference with much fewer transmit antennas than other CEP techniques in the literature. The proposed solution is suitable for PSK as well as multi-level constellations. A more detailed explanation of this work will be presented in Section V-F.

\section{B. Antenna Selection $(A S)$}

From the descriptions for CEP schemes, it is observed that $\mathrm{CE}$ transmission attempts to reduce the hardware complexity and the consequent power consumption at the BS by employing power-efficient and low-cost RF amplifiers. In addition to the CE transmission, AS is also a low-cost and low-complexity alternative for power-efficient and cost-efficient BSs with large-scale antenna arrays, which has received continuous research attention [240], [241]. In AS techniques, only a subset of the entire antenna array is activated for transmission or reception, as shown in Fig. 10b, which allows for a reduction in the number of active RF chains and consequently a reduction in the power consumption. In addition, AS also benefits from exploiting the degrees of freedom provided by the excess of antennas, i.e., antenna diversity at the BS [240], [241].

AS techniques have already been a popular research topic in small-scale MIMO systems [242]-[247], which exhibit benefits in terms of power efficiency [242]. The initial AS approach is based on the exhaustive search method [243], whose computational cost could become impractically high when the number of antennas scales up. For AS at the receiver side, a lowcomplexity AS scheme that maximizes the channel capacity has been introduced in [244], which is aimed at minimizing the performance loss caused by reducing the number of active antennas at the receiver side. Meanwhile, AS methods at the transmitter side have been studied in [245]-[247], where it is shown in [245] that AS can maximize the received SNR when a maximum ratio combining (MRC) detector is considered at the receiver. [246] and [247] further reveal that the error rate performance can be further improved by designing transmit AS techniques. More recently, research on AS has been extended to large-scale antenna arrays in [248]-[250], where the energy efficiency benefits offered by AS techniques are shown in [248] and [249]. [250] further reveals that a simple random AS scheme can significantly improve the energy efficiency performance of the BS with a large-scale antenna array.

To extend the concept of CI exploitation to AS techniques, a transmit AS scheme has been designed in [251] to minimize the error rate as an initial attempt, where a partial sub-channel orthogonalization is employed to exploit the constructive part of the existing interference while nullifying the destructive part. Building upon [251], a more advanced CI-driven AS technique is introduced in [252] and [253], which maximizes the CI effect for the users by selecting the antenna subset that achieves the highest CI effect. With the proposed AS algorithms, it is shown that the combination of CI-based AS with MRT precoding is able to outperform more complicated ZF precoding with traditional computationally expensive AS schemes. More recently, thanks to the developments of optimization-based CI precoding in [43] and [44], a joint AS and precoding method based on optimization is further proposed in [254] and [255] for interference exploitation, which are superior to [251]-[253]. A mix-integer optimization problem is firstly formulated, which maximizes the minimum CI effect among the users by jointly optimizing the transmit AS decision and the precoded signals, given by [255]

$$
\begin{aligned}
\mathcal{P}_{\mathrm{C}-\mathrm{AS}}^{\mathrm{PSK}}: & \max _{\mathbf{x}, \mathbf{a}} t \\
\text { s.t. } & \mathrm{C} 1: \mathbf{h}_{k}^{\mathrm{T}} \mathbf{x}=\lambda_{k} s_{k}, \forall k \in \mathcal{K} \\
& \mathrm{C} 2:\left[\Re\left(\lambda_{k}\right)-t\right] \tan \theta_{\text {th }} \geq\left|\Im\left(\lambda_{k}\right)\right|, \forall k \in \mathcal{K} \\
& \mathrm{C} 3:\|\mathbf{x}\|_{2}^{2} \leq P_{0} \\
& \mathrm{C} 4:|\operatorname{sgn}(\mathbf{x})|=\mathbf{a} \\
& \mathrm{C} 5: \sum_{n=1}^{N_{\mathrm{T}}} a_{n}=N_{\mathrm{AC}}, a_{n} \in\{0,1\}, \forall n
\end{aligned}
$$

where $\operatorname{sgn}(\cdot)$ is the sign function, and $\mathbf{a}=\left[a_{1}, a_{2}, \cdots, a_{N_{\mathrm{T}}}\right]^{\mathrm{T}}$. C4 and C5 jointly guarantee that only $N_{\mathrm{AC}}$ transmit antennas are active and transmit precoded signals. In [255], three sub-optimal methods are also introduced for the formulated problem $\mathcal{P}_{\mathrm{C}-\mathrm{AS}}^{\mathrm{PSK}}$ to reduce the computational complexity of the joint approach. Similarly, remarkable performance improvements for this CI-based joint AS and precoding have been observed through extensive numerical results in [255], which demonstrates the superiority of interference exploitation in the area of AS.

Compared with the above symbol-level interferenceexploitation AS techniques [251]-[255], another symbol-level AS scheme that aims to minimize the multi-user interference can be mathematically formulated as the following optimization problem [239], [256]

$$
\begin{array}{ll}
\mathcal{P}_{\mathrm{AS}}: & \min _{\mathbf{x}}\|\mathbf{H} \mathbf{x}-\sqrt{\gamma} \cdot \mathbf{s}\|_{2}^{2} \\
\text { s.t. } & \mathrm{C} 1:\|\mathbf{x}\|_{0}=N_{\mathrm{AC}}
\end{array}
$$


where $\gamma$ is the SNR. $\mathcal{P}_{\mathrm{AS}}$ is a linear least-squares problem with an $\ell_{0}$-norm constraint, which is generally non-convex and requires an exhaustive search solution. To obtain a feasible solution with a lower computational cost, $\mathcal{P}_{\mathrm{AS}}$ can be reformulated into a regularized least-squares regression problem, as detailed in [239] and [256].

\section{Hybrid Analog-Digital (AD) Precoding}

Compared with AS techniques which reduce the hardware complexity by reducing the number of active antennas and accordingly reducing the number of active RF chains, another potential technique that also employs a reduced number of $\mathrm{RF}$ chains is the hybrid AD precoding, which has drawn extensive research attention in the past few years [257], [258]. Different from AS techniques, all the antennas are active in hybrid $\mathrm{AD}$ structures, and the signal processing is divided into analog part and digital part, as shown in Fig. 10c, where the analog part usually consists of low-cost phase shifters [259]. Hybrid AD structures have firstly been considered for the future millimeter-wave (mmWave) communications as a promising structure for practical implementation. For mmWave communications, while the small wavelength of mmWave signals allows the use of a large-scale antenna array in a small form factor to combat the severe pathloss [260], dedicating a single RF chain for each antenna element becomes nearly infeasible for mmWave transceivers, since the hardware components working at mmWave bands are costly and power expensive [257]-[259]. By reducing the number of $\mathrm{RF}$ chains employed at the transceivers, hybrid AD structures are able to greatly reduce the hardware complexity and the corresponding power consumption at the cost of only a slight performance loss, thus achieving an improved balance between performance, complexity and cost.

In [261], a single-user mmWave communication system has been considered, and the hybrid precoding and combining are joint designed to maximize the spectral efficiency, where an orthogonal matching pursuit (OMP)-based algorithm is proposed. The hybrid precoding design for a single-user mmWave transmission in [262] considers both the fullyconnected and the partially-connected AD structures, where a manifold optimization based algorithm is proposed based on the alternating minimization framework. A multi-user transmission has further been considered in [263], where the analog precoder/combiner is designed to maximize the effective channel gain while the digital precoder is designed to mitigate the multi-user interference based on $\mathrm{ZF}$, constituting a two-stage hybrid precoding. An important proposition is established in [264], where it is shown that hybrid precoding is able to realize any fully-digital precoding when the number of RF chains is twice the number of data streams. Meanwhile, [264] also proposes a near-optimal hybrid precoding design for both single-user and multi-user transmissions, when the number of RF chains becomes fewer. [265] has specifically focused on the partially-connected structures in a multi-user scenario and proposed hybrid precoding designs based on the concept of successive interference cancellation (SIC). By assuming the digital precoder to be a diagonal matrix, the total spectral efficiency optimization problem is decomposed into a series of simple sub-rate optimization problems, which can be efficiently solved by the power iteration algorithm. Additional works on hybrid precoding include some low-complexity designs based on MRT in [266], virtual path selection in [267], and SVD in [268]. Mathematically, a common multiuser hybrid precoding problem aimed at spectral efficiency maximization can be formulated as [267]

$$
\begin{aligned}
\mathcal{P}_{\mathrm{HAD}}: & \max _{\mathbf{F}_{\mathrm{RF}}, \mathbf{f}_{k}^{\mathrm{BB}}, \mathbf{w}_{k}} \\
\text { s.t. } & \sum_{k=1}^{K} \log _{2}\left(1+\frac{\left|\mathbf{w}_{k}^{\mathrm{H}} \mathbf{H}_{k} \mathbf{F}_{\mathrm{RF}} \mathbf{f}_{k}^{\mathrm{BB}}\right|^{2}}{\sum_{i \neq k}\left|\mathbf{w}_{k}^{\mathrm{H}} \mathbf{H}_{k} \mathbf{F}_{\mathrm{RF}} \mathbf{f}_{i}^{\mathrm{BB}}\right|^{2}+\sigma^{2}}\right) \\
& \mathrm{C} 1: \mathbf{F}_{\mathrm{RF}} \in \mathcal{F}, \mathbf{w}_{k} \in \mathcal{W}, \forall k \in \mathcal{K} \\
& \mathrm{C} 2:\left\|\mathbf{F}_{\mathrm{RF}}\left[\mathbf{f}_{1}^{\mathrm{BB}}, \mathbf{f}_{2}^{\mathrm{BB}}, \cdots, \mathbf{f}_{K}^{\mathrm{BB}}\right]\right\|_{F}^{2}=P_{0}
\end{aligned}
$$

which assumes single-stream transmission for each user and analog combining only at the receiver side. The constraint $\mathrm{C} 1$ is to ensure that the analog precoder $\mathbf{F}_{\mathrm{RF}}$ and analog combiner $\mathbf{w}_{k}$ implemented with phase shifters have constant-envelope entries.

If we assume the analog precoder to be fixed when we design the precoding methods for the digital part, hybrid AD structures are equivalent to a fully-digital MIMO system transmitting through an effective analog channel. From this point of view, interference exploitation techniques can be readily applied to hybrid $\mathrm{AD}$ structures for additional performance improvements, as recently shown in [269]-[273]. In [269], the digital part of hybrid precoding employs CI precoding, and several analog precoding designs particularly tailored for CIbased hybrid precoding are presented and compared. It should be noted that since CI-based precoder is data-dependent, the common spectral efficiency expression is not applicable to CIbased hybrid precoding designs. Accordingly, by considering a transmit power minimization problem for CI-based hybrid precoding in a MU-MISO system, the corresponding optimization problem can be formulated as [269]

$$
\begin{aligned}
\mathcal{P}_{\mathrm{C}-\mathrm{HAD}}^{\mathrm{PSK}}: & \min _{\mathbf{F}_{\mathrm{RF}}, \mathbf{x}}\left\|\mathbf{F}_{\mathrm{RF}} \mathbf{x}\right\|_{2}^{2} \\
\text { s.t. } & \mathrm{C} 1: \mathbf{h}_{k}^{\mathrm{T}} \mathbf{F}_{\mathrm{RF}} \mathbf{x}=\lambda_{k} s_{k}, \forall k \in \mathcal{K} \\
& \mathrm{C} 2:\left[\Re\left(\lambda_{k}\right)-\sqrt{\Gamma_{k} \sigma^{2}}\right] \tan \theta_{\mathrm{th}} \geq\left|\Im\left(\lambda_{k}\right)\right|, \forall k \in \mathcal{K} \\
& \mathrm{C} 3: \mathbf{F}_{\mathrm{RF}} \in \mathcal{F}
\end{aligned}
$$

where $\mathbf{F}_{\mathrm{RF}}$ is updated on a block level and $\mathbf{x}$ is updated on a symbol level. This problem is generally non-convex because of the constant-envelope constraint for the entries in $\mathbf{F}_{\mathrm{RF}}$. Therefore, [269] decomposes the joint design into the analog precoding design, followed by the digital precoding design. As a step further, a CI-based hybrid precoding that is specifically designed to be robust against phase errors in the phase shifters is proposed in [270], where the optimal robust digital precoding is obtained based on the cutting plane method and alternating procedure, when the analog part of the hybrid precoder is fixed. Significant performance improvements in terms of SER can be observed for CI-based hybrid designs 
compared with traditional hybrid methods. Additional studies upon the above existing works include [271] and [272] that extend hybrid precoding to tunable MIMO scenarios, as well as [273] that combines hybrid precoding with low-resolution DACs.

\section{Low-Resolution DACs}

In addition to the use of low-cost RF amplifiers as in CEP and the activation of a reduced number of RF chains as in AS and hybrid precoding, another potential technique to reduce the cost and power consumption per RF chain at the BSs is to employ low-resolution DACs [274], as shown in Fig. 10d. It is known that the power consumption of DACs grows linearly with the bandwidth and exponentially with the resolution [274], and each transmit signal is generated by a pair of DACs connected to the RF chain. Given hundreds of antenna elements at a large-scale antenna array, a large number of DACs are also required, which poses a significant practical challenge if high-resolution DACs are deployed. Therefore, the use of low-resolution DACs, especially the extreme case 1-bit DACs, can greatly simplify the hardware cost and the corresponding power consumption at the BS. In addition, the output signals of 1-bit DACs are CE signals, which allows the use of power-efficient amplifiers to further reduce the hardware complexity.

There have been an increasing number of studies on the downlink transmission design with low-resolution DACs. To be more specific, linear precoding methods with few-bit DACs for downlink MIMO systems have firstly been studied in [275]-[277], where due to the coarse quantization, significant performance degradation is observed compared with the ideally unquantized case, especially when 1-bit DACs are considered. Nonlinear precoding designs, which directly design the precoded signals based on the CSI and the data symbols, have further been studied in [29], [278]-[284], and the optimization problem can be formulated as [29]

$$
\begin{aligned}
\mathcal{P}_{\mathrm{DAC}}: & \min _{\mathbf{x}}\left\|\mathbf{s}-\beta_{\mathrm{DAC}} \cdot \mathbf{H x}\right\|_{2}^{2}+K \beta_{\mathrm{DAC}}^{2} \sigma^{2} \\
\text { s.t. } & \mathrm{C} 1: \mathbf{x} \in \mathcal{X}_{\mathrm{DAC}} \\
& \mathrm{C} 2: \beta_{\mathrm{DAC}}>0
\end{aligned}
$$

which aims to minimize the MSE between the transmit data symbols and the received symbols. $\mathcal{X}_{\mathrm{DAC}}$ is the set consisting of the output signals for low-resolution DACs, and in the case of 1-bit DACs, $\mathcal{X}_{\mathrm{DAC}}=\left\{ \pm \sqrt{\frac{P_{0}}{2 N_{\mathrm{T}}}} \pm \sqrt{\frac{P_{0}}{2 N_{\mathrm{T}}}} \cdot j\right\}$. In [278], a non-linear precoding method based on the biconvex relaxation framework is proposed for $\mathcal{P}_{\mathrm{DAC}}$, which achieves a promising performance with a low computational cost. Its corresponding very large-scale integration (VLSI) design architectures have further been illustrated in [279] to showcase the efficacy. [280] proposes several 1-bit precoding schemes based on SDR, sphere encoding, and squared $\ell_{\infty}$-norm relaxation. Meanwhile, a 1-bit precoding method is described in [281] based on the branch-and-bound framework, which can theoretically achieve the optimal performance. Some other downlink precoding designs for low-resolution DACs include SER minimization in [282]-[284] and alternating minimization in [29]. A general observation is that non-linear precoding designs can achieve a significantly better performance than the linear methods, when low-resolution DACs are employed at the transmitter.

To achieve a promising error rate performance, the precoding designs for 1-bit DACs have to be non-linear and exploit the information of the data symbols, which creates the opportunity for interference exploitation, as recently studied in [69] and [285]-[287]. [285] considers the transmit signal design for 1-bit massive MIMO system based on CI optimization, where the CI effect is maximized subject to the output constraints of DACs, and the corresponding optimization problem is constructed as [285]

$$
\begin{aligned}
\mathcal{P}_{\mathrm{C}-\mathrm{DAC}}^{\mathrm{PSK}}: & \max _{\mathbf{x}} t \\
\text { s.t. } & \mathrm{C} 1: \mathbf{h}_{k}^{\mathrm{T}} \mathbf{x}=\lambda_{k} s_{k}, \forall k \in \mathcal{K} \\
& \mathrm{C} 2:\left[\Re\left(\lambda_{k}\right)-t\right] \tan \theta_{\text {th }} \geq\left|\Im\left(\lambda_{k}\right)\right|, \forall k \in \mathcal{K} \\
& \mathrm{C} 3: \mathbf{x} \in \mathcal{X}_{\mathrm{DAC}}
\end{aligned}
$$

In the case where 1-bit DACs are employed, by expressing $\mathcal{P}_{\mathrm{C}-\mathrm{DAC}}^{\mathrm{PSK}}$ into a real representation and relaxing the 1bit constraint, the formulated optimization problem is shown to be a linear programming (LP), which can be efficiently solved, and the final transmit signal is obtained by enforcing an element-wise normalization. [69] and [286] focus on the 1-bit precoding designs for PSK modulations based on the symbol-scaling CI metric, and a refinement process that is applicable upon any 1-bit schemes has been introduced, where additional performance improvements can be observed. More recently, [287] proposes a near-optimal 1-bit precoding design for both PSK and QAM signaling based on the partial branchand-bound (BB) approach, and the performance improvements are shown to be significant compared with other existing 1bit precoding designs. A joint consideration of hybrid $\mathrm{AD}$ precoding and 1-bit DACs based on interference exploitation has further been studied in [273], where it is shown that compared with hybrid AD structures with ideal DACs, the number of RF chains in the presence of 1-bit DACs has to be much larger than the number of data streams to achieve a near-optimal performance.

In addition to the above studies that specifically focus on 1bit DACs, [288] has discussed the extension from 1-bit DACs to few-bit DACs, where the objective function adopts (71). In the case when $B$-bits DACs are adopted at the BS, the set $\mathcal{X}_{\text {DAC }}$ can be expressed as [288]

$$
\mathcal{X}_{\mathrm{DAC}}=\left\{ \pm \sqrt{\frac{P_{0}}{2 N_{\mathrm{T}}}}, \cdots, \pm \sqrt{\frac{P_{0}}{2^{B-2} N_{\mathrm{T}}}} \pm \sqrt{\frac{P_{0}}{2^{B-1} N_{\mathrm{T}}}}\right\},
$$

which is obtained via normalizing the outputs of a uniform quantizer such that the transmission power constraint is always satisfied. Similar to the case of 1-bit DACs, the resulting optimization problem in the presence of $B$-bits DACs is NP-hard, and the optimal solution obtained via an exhaustive search method is prohibitive in terms of computational complexity for large-scale antenna arrays. To obtain a feasible solution, [288] has developed a cyclic coordinate descent (CCD) algorithm [289] with the effort to provide an efficient solution for this problem. It has been shown in [288] that the complexity of 
the proposed algorithm is only $\mathcal{O}\left(N_{\mathrm{T}}^{2} 2^{B}\right)$ which is much smaller compared with the $\mathcal{O}\left(N_{\mathrm{T}} 2^{B N_{\mathrm{T}}}\right)$ of the exhaustive search approach.

\section{E. Non Linearities}

As already mentioned in Section V-A, it is common to employ power amplifiers characterized by severe non-linear effects in practical systems relying on large-scale antenna arrays at the transmitter. Therefore, good dynamic properties of the per-antenna transmit power are required in order to limit such effects. In this context, the discussed CEP schemes are an effective strategy, since they achieve the best possible PAPR (unit) at the symbol level. Meanwhile, alternative schemes based on SLP have also been proposed in the literature for non-linear channels in [53], [290]-[295], where the aim is not to attain a $\mathrm{CE}$ transmission, but rather to optimize the power dynamics (such as the dynamic range and PAPR) following an optimization framework in line with [42], [222] based on per-user QoS constraints. The main idea of these schemes is to control the instantaneous transmit power and to minimize its peaks, both in the temporal dimension and in the spatial one (i.e. among different antenna elements), so as to limit the performance degradation due to the amplitude-to-amplitude (AM-AM) and the amplitude-to-phase (AM-PM) distortion. An example of AM-AM and AM-PM characteristics of a non-linear amplifier [296] is shown in Figs. 11a and 11b, respectively. In particular, Fig. 11a highlights how the temporal variation of the power around the operating point enhances the distortion. On the other hand, Fig. 11b shows that the spatial variation of the instantaneous power (across different antennas) also leads to signal deterioration through a differential phase shift.

A SLP approach for non-linear systems, proposed in [53], considers a weighted per-antenna PM problem, subject to QoS constraints and a lower bound constraint on the per-antenna transmit power. In this scheme, the imbalances between different RF chains are reduced by constraining the per-antenna transmit power within a specific range. More specifically, the goal is to minimize the maximum power among the different antennas, and meanwhile put a lower bound constraint on such power. The related optimization problem tailored for APSK modulations can be constructed as [53]

$$
\begin{aligned}
\mathcal{P}_{\mathrm{NL}}^{\mathrm{APSK}}: & \min _{\mathbf{x}} r \\
\text { s.t. } & \mathrm{C} 1: \alpha r^{2} \leq \frac{\left|x_{i}\right|^{2}}{p_{i}} \leq r^{2}, \forall i \in\left\{1,2, \cdots, N_{t}\right\} \\
& \mathrm{C} 2:\left|\mathbf{h}_{k}^{\mathrm{T}} \mathbf{x}\right|^{2} \geq \kappa_{k}^{2} \gamma_{k} \sigma^{2}, \forall k \in \mathcal{K} \\
& \mathrm{C} 3: \angle \mathbf{h}_{k}^{\mathrm{T}} \mathbf{x}=\angle s_{k}, \forall k \in \mathcal{K}
\end{aligned}
$$

where the parameter $\alpha$, chosen such that $0 \leq \alpha \leq 1$, determines the lower bound. The closer $\alpha$ is to 1 , the more the power variations will be limited. Nonetheless, the choice of a higher value for $\alpha$ also results in a reduction of the degrees of freedom of the optimization problem. This has been solved through an iterative procedure based on successive convex approximation (SCA). It shall be stressed that this scheme optimizes the power dynamics in the spatial dimension (across

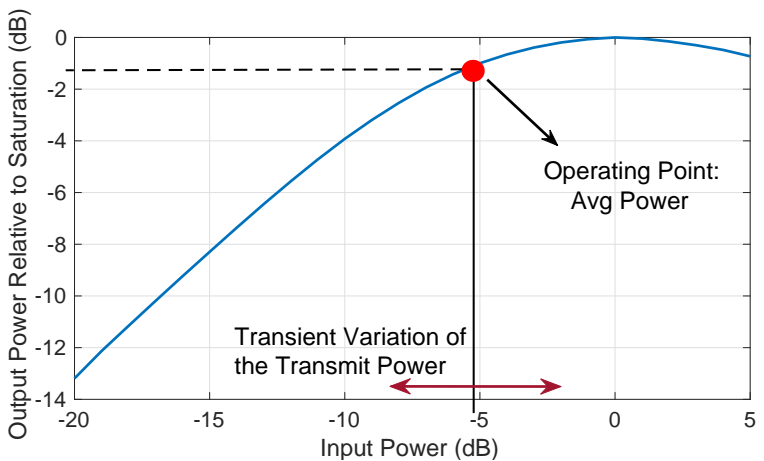

(a) AM-AM

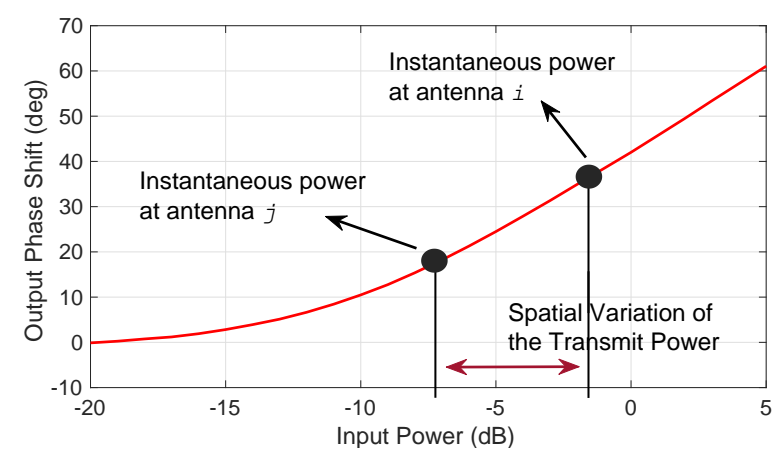

(b) AM-PM

Fig. 11: An example of the AM-AM/AM-PM characteristic of a non-linear amplifier

different antenna elements) only, while not in the temporal one, as the optimization is performed symbol by symbol.

A second strategy to improve the spatial dynamics of the transmitted signals is proposed in [53], [290], which performs a minimization on the spatial PAPR, evaluated amongst the transmitting antennas, under QoS constraints. The related optimization problem is formulated as a non-linear fractional programming, given by [290]

$$
\begin{aligned}
\mathcal{P}_{\mathrm{NL}}^{\mathrm{PAPR}}: & \min _{\mathbf{x}} \frac{\|\mathbf{x}\|_{\infty}^{2}}{\|\mathbf{x}\|_{2}^{2}} \\
\text { s.t. } & \mathrm{C} 1:\left|\mathbf{h}_{k}^{\mathrm{T}} \mathbf{x}\right|^{2} \geq \kappa_{k}^{2} \gamma_{k} \sigma^{2}, \forall k \in \mathcal{K} \\
& \mathrm{C} 2: \angle \mathbf{h}_{k}^{\mathrm{T}} \mathbf{x}=\angle s_{k}, \forall k \in \mathcal{K}
\end{aligned}
$$

which is tackled by resorting jointly to parametric programming and SCA. Both the introduced schemes above have been shown to outperform the state-of-the-art SLP schemes based on QoS constraints optimization, in terms of spatial PAPR, spatial dynamic range, and SER over non-linear channels. While the power minimization scheme with a lower bound constraint has been shown to be more flexible than the spatial PAPR minimization one, the latter is able to achieve a slightly lower SER.

Finally, a spatio-temporal extension of the SLP PAPR minimization approach has been proposed in [291], which is based on the system model introduced in Section IV-F for FTN signaling. This allows a minimization of the PAPR at a waveform level, both in spatial domain and in temporal domain. The associated optimization formulation is formally 
similar to the one in (75), which is thus tackled analogously. This spatio-temporal SLP scheme for non-linear channels has been shown to achieve considerable performance gains with respect to the previous ones, in terms of power distribution and SER over non-linear channels.

\section{F. Single-RF MIMO and RF-domain SLP}

In addition to hardware-efficient structures mentioned in the previous sections, more recently [239] has proposed two new transmitter architectures, illustrated in Fig. 12a and Fig. $12 \mathrm{~b}$, respectively. These two hardware structures deal with the increased hardware complexity and power consumption of existing techniques by eliminating the need for DACs and replacing them with analog components. In the proposed transmitter designs, which are referred to as RF-domain SLP, the processing happens only in the RF domain, as the DACs have been eliminated. The transmitted signals are modulated directly on the antennas by analog phase shifters.

The first architecture in Fig. 12a includes a single variable gain amplifier (VGA), which controls the amplitude $\alpha$ of the signals that are driven to the transmit antennas, where each antenna is driven by a dedicated phase shifter that changes the phase of the RF signal before transmission. As a result, the transmit signal can be expressed as [239]

$$
x_{n}=\alpha e^{j \theta_{n}}, \forall n \in\left\{1,2, \cdots, N_{\mathrm{T}}\right\} .
$$

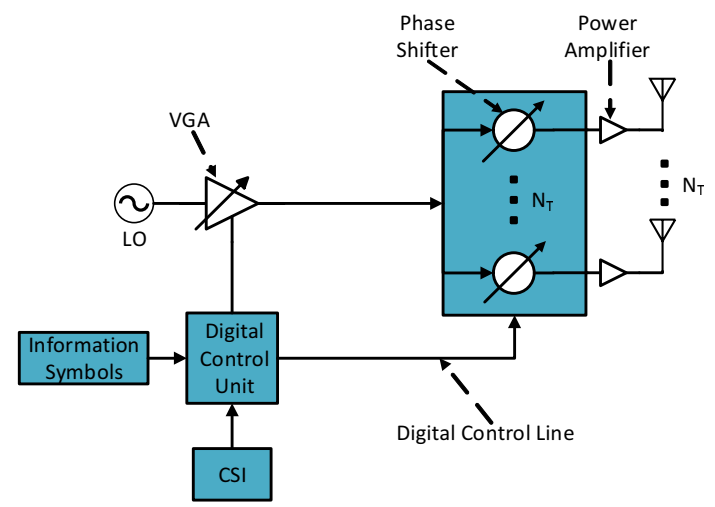

(a) 1-PS per antenna at the transmitter

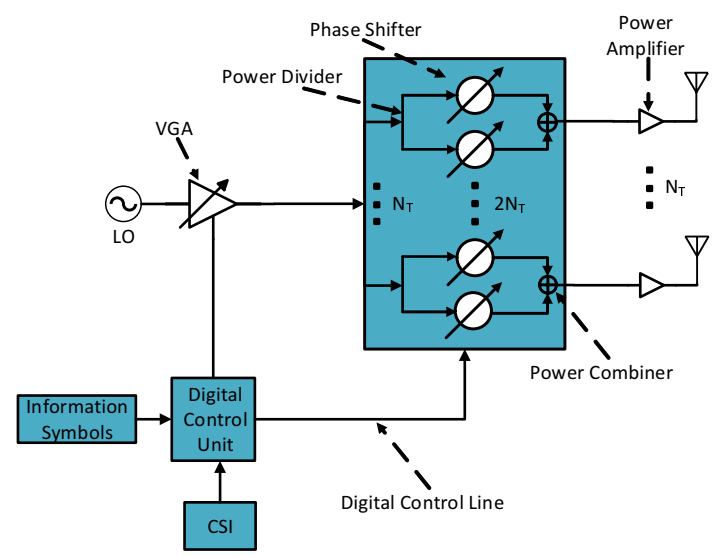

(b) 2-PS per antenna at the transmitter

Fig. 12: Block diagram of the RF-domain SLP
The purpose of the precoding is to find each phase shift $\theta_{n}$ and the gain of the VGA $\alpha$ that minimize the Euclidean distance between the received signal at the user side and the information symbol. Accordingly, the problem can be formulated as [239]

$$
\begin{aligned}
\mathcal{P}_{\mathrm{DM}}^{\mathrm{VGA}}: & \min _{\mathbf{v}, \alpha}\|\alpha \cdot \mathbf{H v}-\sqrt{\gamma} \cdot \mathbf{s}\|_{2}^{2} \\
\text { s.t. } & \mathrm{C} 1:\left|v_{n}\right|=1, \forall n \in\left\{1,2, \cdots, N_{\mathrm{T}}\right\}
\end{aligned}
$$

where $\mathbf{v}$ is an auxiliary variable such that $\mathbf{x}=\alpha \cdot \mathbf{v}$. An iterative solution based on the coordinate descent algorithm has been developed in [239] to solve this non-convex problem, which is shown via numerical results to converge to a local minima. As already mentioned in Section V-A, the derived solution for this architecture is also an efficient solution for CEP, when the VGA gain $\alpha$ is kept constant regardless of the channel realization $\mathbf{H}$ and the information symbols $\mathbf{s}$.

Similar to [297], an alternative transmitter structure has also been proposed in [239], as shown in Fig. 12b, where each antenna element adopts a dual phase shifter structure. With the joint effect of two phase shifters, the amplitude of the transmit signal can also be altered by selecting suitable phases for the two superimposing signals. Therefore, the transmit signal $x_{n}$ on the $n$-th antenna port can be expressed as [239]

$$
x_{n}=\alpha\left(e^{j \theta_{n, 1}}+e^{j \theta_{n, 2}}\right), \forall n \in\left\{1,2, \cdots, N_{\mathrm{T}}\right\} .
$$

The objective of the precoder remains the same, while the constraint has to adapt to the addition of the second phase shifter. Subsequently, the precoding problem is formulated as [239]

$$
\begin{aligned}
\mathcal{P}_{\mathrm{DM}}^{\mathrm{DPS}}: & \min _{\mathbf{v}, \alpha}\|\alpha \cdot \mathbf{H v}-\sqrt{\gamma} \cdot \mathbf{s}\|_{2}^{2} \\
\text { s.t. } & \mathrm{C} 1:\|\mathbf{v}\|_{\infty} \leq 2, \forall n \in\left\{1,2, \cdots, N_{\mathrm{T}}\right\}
\end{aligned}
$$

This optimization problem can be solved using an iterative solution based on the coordinate descent method, while with a different update step compared with that of $\mathcal{P}_{\mathrm{DM}}^{\mathrm{VGA}}$, as detailed in [239]. Numerical results show that the addition of a second phase shifter provides an interesting tradeoff, as a small increase in the power consumption is observed due to the increased number of phase shifters, while a decrease in the computational complexity of the solution is also observed, since the set of constraints of the optimization problem now becomes convex.

The proposed RF-domain systems, where DACs are replaced by analog components, are shown to outperform competing fully-digital and hybrid $\mathrm{AD}$ structures when the number of transmit antennas is much larger than that of users, thanks to the low power consumption of the analog phase shifters. More specifically, it has been shown in numerical results that for a system with 100 transmit antennas and 10 singleantenna users, the RF-domain SLP method with 1-PS and 2PSs can achieve $36 \%$ and $11 \%$ improvement in terms of power efficiency over the hybrid AD precoding, and $135 \%$ and $83 \%$ over the fully-digital SLP scheme, respectively.

\section{G. Summary, Insights and Lessons Learnt}

Large-scale antenna arrays are indispensable components for $5 \mathrm{G}$ and future wireless communication systems, while current hardware-efficient large-scale antenna architectures have 
TABLE IV: A summary for the application of CI-based techniques in various wireless scenarios

\begin{tabular}{|c|c|c|c|c|}
\hline Reference & Considered Scenario & CI Metric & Modulation & CSI Assumption \\
\hline $\begin{array}{c}{[131]} \\
{[132]} \\
{[135]} \\
{[120],[133]}\end{array}$ & $\mathrm{CR}$ & $\begin{array}{c}\text { Selective CI } \\
\text { Correlation-rotation CI } \\
\text { Strict phase rotation } \\
\text { Non-strict phase rotation }\end{array}$ & PSK & Perfect \\
\hline$[153]-[155]$ & SWIPT & Non-strict phase rotation & PSK & Perfect \\
\hline$[171]$ & SWIPT + PHY security & Non-strict phase rotation + Symbol scaling & $\mathrm{PSK}+\mathrm{QAM}$ & Perfect + Imperfect \\
\hline $\begin{array}{c}167],[168] \\
{[169]} \\
{[170]}\end{array}$ & PHY security & $\begin{array}{c}\text { Strict phase rotation }+ \text { Non-strict phase rotation } \\
\text { Non-strict phase rotation }\end{array}$ & PSK & $\begin{array}{c}\text { Perfect } \\
\text { Perfect }+ \text { Imperfect } \\
\text { Perfect }\end{array}$ \\
\hline $\begin{array}{l}{[188]} \\
{[189]}\end{array}$ & FD communications & $\begin{array}{c}\text { Non-strict phase rotation } \\
\text { Non-strict phase rotation }+ \text { Symbol Scaling }\end{array}$ & $\begin{array}{c}\text { PSK } \\
\text { PSK + QAM }\end{array}$ & $\begin{array}{c}\text { Perfect } \\
\text { Perfect }+ \text { Imperfect }\end{array}$ \\
\hline [190] & FD communications + SWIPT & Non-strict phase rotation & PSK & Imperfect \\
\hline $\begin{array}{l}{[213]} \\
{[214]}\end{array}$ & $\begin{array}{c}\text { Multi-cell DA + PHY security + AS } \\
\text { Multi-cell DA }\end{array}$ & Non-strict phase rotation & PSK & Imperfect \\
\hline $\begin{array}{c}{[220]} \\
{[219],[221]}\end{array}$ & Spatio-Temporal CI & $\begin{array}{l}\text { Non-strict phase rotation } \\
\text { Symbol scaling }\end{array}$ & $\begin{array}{l}\text { PSK } \\
\text { QAM }\end{array}$ & Perfect \\
\hline $\begin{array}{c}{[235]-[237]} \\
{[238]}\end{array}$ & CE precoding & $\begin{array}{c}\text { Non-strict phase rotation } \\
\text { Non-strict phase rotation }+ \text { Symbol scaling }\end{array}$ & $\begin{array}{c}\text { PSK } \\
\text { PSK + QAM }\end{array}$ & Perfect \\
\hline $\begin{array}{l}{[251]-[253]} \\
{[254],[255]}\end{array}$ & $\begin{array}{c}\text { Transmit AS } \\
\text { Transmit AS + Precoding }\end{array}$ & $\begin{array}{c}\text { Selective CI } \\
\text { Non-strict phase rotation }\end{array}$ & PSK & $\begin{array}{c}\text { Perfect } \\
\text { Perfect }+ \text { Imperfect }\end{array}$ \\
\hline $\begin{array}{c}{[269],[270]} \\
{[63]}\end{array}$ & $\begin{array}{c}\text { Hybrid precoding } \\
\text { Hybrid precoding }+ \text { Tunable load }\end{array}$ & $\begin{array}{c}\text { Non-strict phase rotation } \\
\text { Non-strict phase rotation }+ \text { Symbol scaling }\end{array}$ & $\begin{array}{c}\text { PSK } \\
\text { PSK + QAM }\end{array}$ & Perfect \\
\hline [273] & Hybrid precoding + 1-bit DACs & Symbol scaling & PSK & Perfect \\
\hline $\begin{array}{c}{[285]} \\
{[69],[286]} \\
{[287]} \\
\end{array}$ & 1-bit DACs & $\begin{array}{c}\text { Non-strict phase rotation } \\
\text { Non-strict phase rotation + Symbol scaling } \\
\text { Symbol scaling }\end{array}$ & $\begin{array}{c}\text { PSK } \\
\text { PSK + QAM }\end{array}$ & Perfect \\
\hline [53], [290], [291] & Non-linear channels & Strict phase rotation & APSK & Perfect \\
\hline
\end{tabular}

to be adopted for both energy efficiency and cost efficiency. Since hardware-constrained large-scale antenna arrays introduce additional non-negligible interference due to imperfect hardware components, the major benefits of exploiting CI for in these areas are the significant performance improvements compared with interference-reduction approaches, as summarized below.

For CEP, CI-based designs lead to both simplification in problem formulation and additional performance improvements. Compared with traditional interference-reduction CEP approaches that need to resort to complex CEO method, it is shown that a simple two-step convex approach based on the CI formulation can already lead to improved error rate performance [236]. By further considering the CI-based CEP problem from the perspective of Riemannian manifold, it is shown in [237] that additional performance improvements can be obtained. Both of the above results exhibit the benefits of exploiting interference in precoding designs for $\mathrm{CE}$ transmission.

Due to the nature of AS, the optimal AS strategy can only be obtained via the exhaustive search method, which is not applicable due to its expensive computational cost, and therefore existing AS approaches can only pursue sub-optimal solutions. Thanks to the capability of exploiting interference, it is shown that CI-AS with MRT precoding can outperform traditional AS schemes with ZF precoding. By combining AS with more complicated optimization-based CI formulation, which leads to a mix-integer optimization problem, remarkable performance improvements can be observed for this joint ASprecoding method.

Similar to the case of AS, the optimal solution to 1bit precoding needs an exhaustive search process, which is prohibitive. Nevertheless, CI-based techniques are shown to be quite effective in the area of low-resolution DACs, as proposed in [69] where computationally-efficient solutions are obtained by reformulating the relaxed 1-bit precoding problem into a LP optimization. For both CEP and 1-bit DACs, it is interesting to observe that while the corresponding strict constraints have been relaxed to arrive at a convex formulation, most of the entries of the solution to the relaxed optimization problems already satisfy the CE constraints or the 1-bit constraints, which demonstrate the superiority of exploiting interference in these scenarios. Moreover, building upon this observation, a near-optimal CEP algorithm and 1-bit precoding design have been proposed based on the BB framework in [298] and [287], respectively. 
As a step further towards practical implementation, Section V-E has discussed the SLP techniques that pursue low-PAPR transmission by controlling the instantaneous transmit power and minimizing the peaks. Moreover, the single-RF MIMO architecture, as discussed in Section V-F, is a new hardwareefficient transmit multi-antenna design, which removes the DACs and replaces them with analog components, where a single VGA is included. Thus, the signal processing happens only in the RF domain and the transmit signals are modulated by phase shifters. Both of the above architectures can be seen as extensions of CEP. For low-PAPR transmission, the strict ' $\mathrm{CE}$ ' requirement is replaced by a constraint that limits the transmit signal on each antenna port to be not larger than a pre-defined threshold, while for single-RF MIMO a scaling on the CE signals is allowed due to the inclusion of the VGA.

For convenience, we summarize the CI-based techniques for the wireless application scenarios discussed in Section IV and Section V in Table IV.

\section{Proof-OF-Concept Testbeds}

Section III - Section V have reviewed how CI precoding can benefit various wireless communication scenarios from the perspective of theoretical studies. In order to further advocate the use of CI-based SLP techniques in practical wireless communication systems, in this section we illustrate the potential gains of exploiting CI in practical systems by describing two testbeds that are currently available to perform CI precoding, hosted in University College London (UCL) and University of Luxembourg (UL), respectively.

\section{A. Multi-Carrier CI Testbed}

A multi-carrier proof-of-concept hardware platform is hosted in UCL, which employs real-time CI precoding.

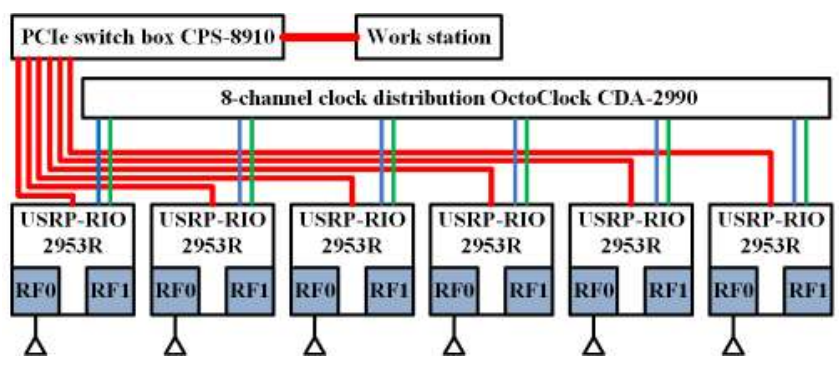

Fig. 13: System architecture of the real-time hardware platform

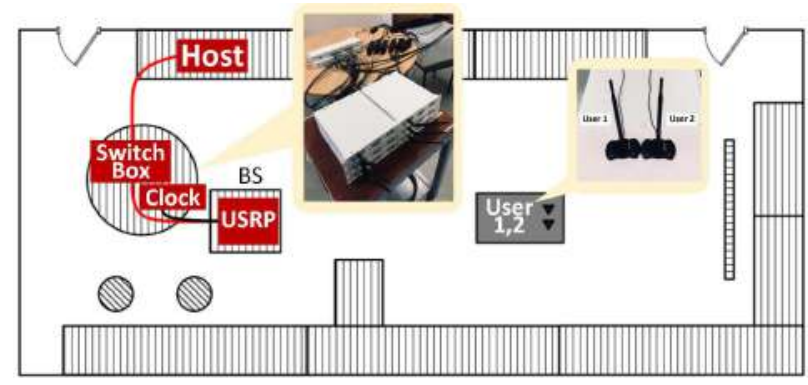

Fig. 14: Illustration of the indoor experimental environment
The platform combines CI precoding with the spectral efficient frequency division multiplexing (SEFDM) [300], [299]. Compared with orthogonal frequency division multiplexing (OFDM), SEFDM breaks the orthogonality by allowing closely-spaced non-orthogonal sub-carriers such that the total occupied bandwidth is reduced, thereby improving the effective spectral efficiency. Meanwhile, this artificial non-orthogonal setting creates inter-carrier interference (ICI), which can be exploited by CI precoding, as studied in [300].

1) Platform Description: The system architecture of the real-time hardware platform is presented in Fig. 13, where the experiment configurations follow the 3GPP NB-IoT standard [301]. In this testbed, omni-directional antennas are used for both the transmitter and receiver to validate the feasibility of precoding. A total number of 6 transmit antennas are configured for the purpose of demonstration, and accordingly 6 software defined ratio USRP-RIO 2953R devices are connected as a 6-antenna BS. Each USRP has two separate and independent RF chains, where one RF chain is for signal transmission and the other one is for signal reception, as seen in Fig. 13. The raw data stream is generated by the work station, which are then sent to a cabled PCI-Express switch box CPS-8910 via an NI MXI-Express Gen $2 \times 8$ cable that supports a data rate up to $3.2 \mathrm{~Gb} / \mathrm{s}$. The switch box separates the raw data stream into 6 data streams, which are delivered to 6 USRPs in parallel also via the NI MXI-Express cable. For the purpose of synchronization, these USRPs are also connected via SMA cables to a CDA-2990 8-channel clock distribution OctoClock module, which can split and amplify a $10 \mathrm{MHz}$ reference signal and a pulse-per-second (PPS) signal to support synchronization for a maximum of 8 USRPs.

This real-time platform operates at $2.4 \mathrm{GHz}$, and the other key parameters are shown in Table V, where the SEFDM approach with a bandwidth compression ratio $v=0.85$ is considered [300]. The experimental environment is an indoor scenario, as shown in Fig. 14, there are a total number of 2 single-antenna receivers that are deployed and placed randomly, where the distance between the BS and the users is flexible and can be up to 9 meters. Due to this limited indoor space, in addition to the over-the-air experiments, a commercialized channel emulator Spirent VR5 [302] is further adopted in order to compare precoding techniques at different SNR values. Assuming that the noise power mainly comes from the receiver side, the VR5 is connected to the 2 users

TABLE V: Parameters of the Experiment System

\begin{tabular}{|l|c|}
\hline Parameter & Value \\
\hline Number of transmit antennas & 6 \\
Number of users & 2 \\
RF center frequency $(\mathrm{GHz})$ & 2.4 \\
Sampling frequency $(\mathrm{MHz})$ & 1.92 \\
FFT size & 128 \\
Number of guard band sub-carriers & 58 \\
Number of data sub-carriers & 12 \\
Number of cyclic prefix samples & 10 \\
Modulation & QPSK \\
Bandwidth compression ratio $(v)[300]$ & 0.85 \\
Sub-carrier bandwidth $(\mathrm{kHz})$ & 15 \\
Sub-carrier spacing & $15 \times v$ \\
Maximum spectral efficiency (bits/s/Hz) & $2 / v$ \\
\hline
\end{tabular}




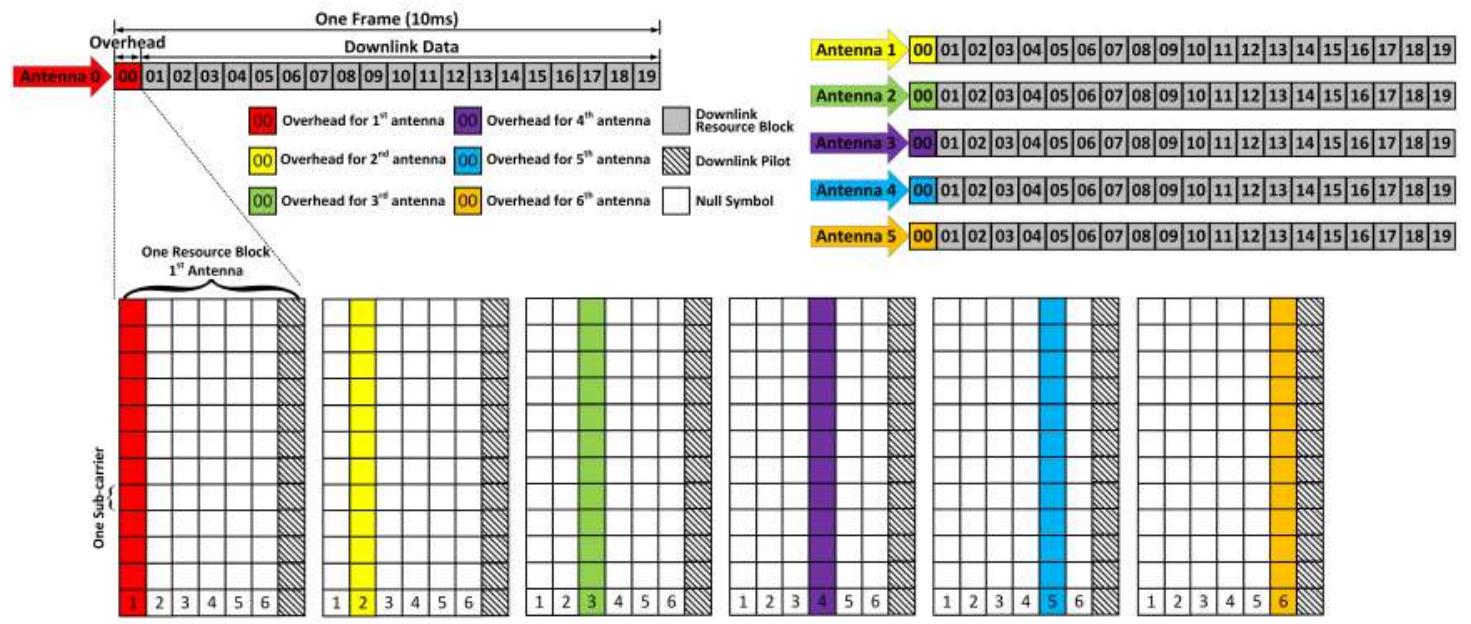

Fig. 15: Frame and resource block structure for each antenna element [299]

via two separate cables. By configuring the VR5, the path loss and the power of the additive Gaussian noise can be set for each user. The receiver bandwidth is set to be $180 \mathrm{kHz}$ and the bandwidth of the additive noise is set to be $1.5625 \mathrm{MHz}$.

2) Frame Structure and CSI Estimation: The transmit signal is generated at the BS with a designed frame structure and resource block structure [299], as shown in Fig. 15. The 10ms frame is divided into 20 time slots with each occupying $0.5 \mathrm{~ms}$, and each time slot consists of 7 OFDM/SEFDM symbols. The first time slot is reserved for CSI estimation, while all the other time slots are used for data transmission. In this realtime platform, the BS obtains the downlink CSI by feedback from the receivers. In order to obtain an accurate estimate of the downlink spatial CSI for each antenna, time-domain orthogonal sounding reference signals (SRSs) are used in the 1 st time slot of each frame. Since the considered system is a $2 \times 6$ MU-MISO scenario, each user only needs to feedback 6 CSI coefficients, which is a reasonable overhead length.

To avoid interference between antennas during CSI estimation, the overhead of CSI estimation for each antenna is allocated at different symbol locations in time, as seen in Fig. 15. More specifically, the overhead of CSI estimation for the first antenna occupies the first OFDM/SEFDM symbol in one resource block, while keeping blank for the following 5 OFDM/SEFDM symbols. With other 5 antennas following the same principle, the overlapping interference can be avoided. The last OFDM/SEFDM symbol in this time slot is reserved for the downlink pilots, which are precoded and then sent simultaneously. These pilot signals are used to compensate for imperfect channel issues such as power normalization and imperfect time and phase synchronization [303].

3) Experimental Validation: The received constellation symbol results that are measured based on over-the-air transmission using this hardware platform are shown in Fig. 15, where the received symbols for the unprecoded system, ZF precoded system and CI precoded system are presented in Fig. 16a, Fig. 16b, and Fig. 16c, respectively. Without precoding, it is observed that the received symbols are scattered in the constellation due to the existence of self-created ICI by SEFDM signals. When $\mathrm{ZF}$ precoding is employed, the distribution of the received symbols becomes more focused at the four nominal QPSK points, as depicted in Fig. 16b, which means that adopting $\mathrm{ZF}$ precoding can improve the performance. When CI precoding is employed, as shown in Fig. 16c, a special received symbol pattern is observed, where we observe that the received symbols are pushed away from the detection thresholds, which follows the design principle of CI precoding. A significantly improved error rate performance can therefore be expected for CI precoded scenario over the unprecoded and $\mathrm{ZF}$ precoded case.

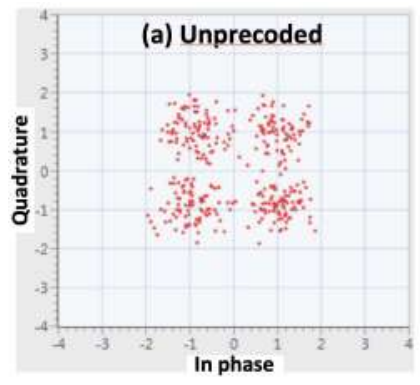

(a) Unprecoded

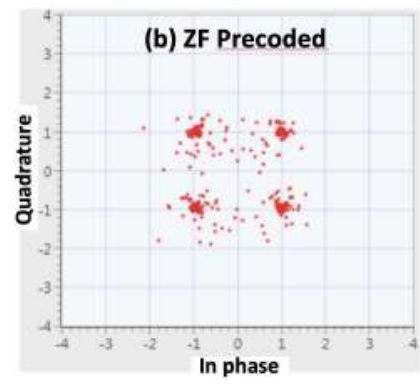

(b) ZF precoded

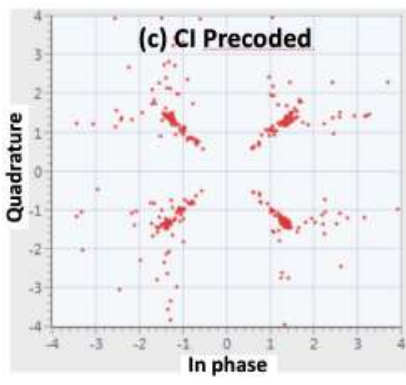

(c) CI precoded

Fig. 16: Experimentally obtained received constellation pattern for unprecoded and precoded system 


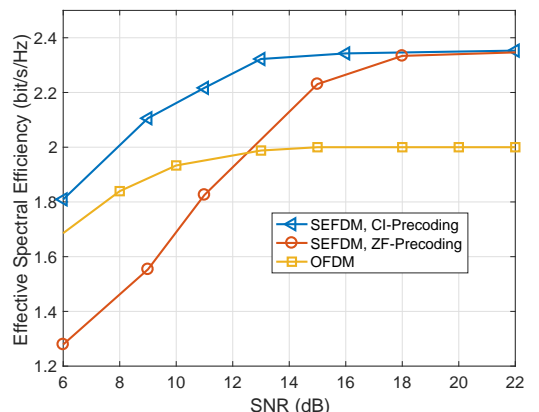

Fig. 17: Experimental result for spectral efficiency, bandwidth compression ratio $v=0.85$

In Fig. 17, the experimental effective spectral efficiency is depicted with an increasing SNR value, where the various SNR values are obtained by tuning the noise power in the VR5 channel emulator. The considered metric, effective spectral efficiency, is calculated as

$$
\mathrm{SE}_{\text {eff }}=\frac{1}{v}(1-\mathrm{BER}) \log _{2} \mathcal{M}
$$

for $\mathcal{M}$-PSK. When QPSK is considered as in the experiment, $\mathcal{M}=4$ and $\mathrm{SE}_{\text {eff }}$ becomes $\mathrm{SE}_{\text {eff }}=\frac{2}{v}(1-\mathrm{BER})$. Based on the result in Fig. 17, we observe that in low-to-medium SNR region, the CI precoded SEFDM system achieves a significantly better performance over the ZF precoded and OFDM case. In the high SNR region, the OFDM case reaches its maximum effective spectral efficiency $2 \mathrm{bits} / \mathrm{s} / \mathrm{Hz}$, because in this case the bandwidth compression ratio is 1 . For the precoding-aided SEFDM results, we observe that both ZF precoded and CI precoded SEFDM approach can reach the maximum effective spectral efficiency $2.35 \mathrm{bits} / \mathrm{s} / \mathrm{Hz}$, both of which outperform the OFDM case.

\section{B. DVB Standard-based CI Testbed}

1) Platform Description: A DVB standard fully-compatible hardware platform is hosted in UL, which employs full frequency reuse in wireless communications named SERENADE [304]. The demonstrator uses the DVB-S2X standard [305] for signal transmission and reception, which includes a novel SLP technique in [82] that optimizes the precoding vectors per every modulated symbol vector, and software defined radios are used to build the testbed. This platform enables the design of a scalable architecture of the transmitter, channel emulator and UEs, as shown in Fig. 18. The commercially available software defined radio platform developed by National Instruments (NI) has been employed for this task. The platform consists of two NI PXI (PCI EXtension for Instruments) 1085 chassis, which allow centralized connection of the set of the NI USRP (Universal Software Radio Peripheral) 2954R and FlexRIO (Reconfigurable IO) 7976R. The NI USRP and FlexRIO have integrated FPGA (Field-Programmable Gate Array) module Kintex-7 from Xilinx.

The transmitter simultaneously transmits 6 precoded signals towards 6 user terminals through a $6 \times 6$ multi-beam channel

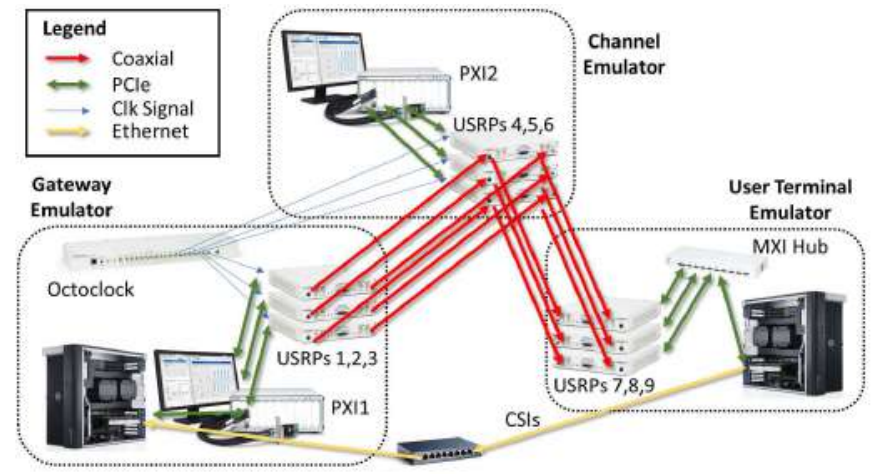

Fig. 18: Implementation platform of the hardware demonstrator

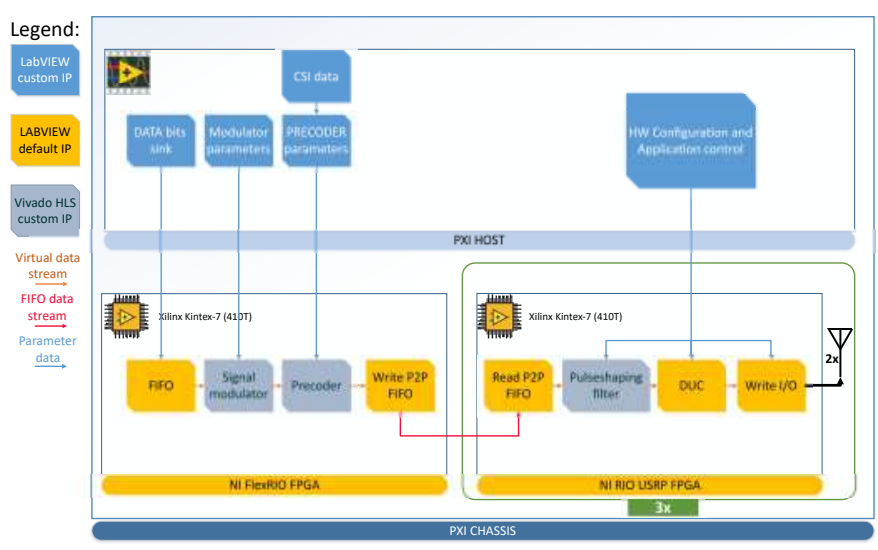

Fig. 19: Block diagram of the transmitter

emulator. The channel emulator acquires the transmitted signals, applies the impairments of the communication channel, Gaussian noise, and the multi-beam interference and transmits the signals to the UEs. Each UE estimates $\mathbf{h}_{k}$ based on the orthogonal Walsh-Hadamard pilot sequences and reports the estimated values to the transmitter through a dedicated feedback channel over an Ethernet link. The transmitter uses this CSI and modulated symbols to compute the precoding matrix. In the full frequency reuse scenario, the multi-user interference is mitigated by precoding techniques. Table VI summarizes the operational parameters of the demonstrator.

The transmitter operates with a central NI FlexRIO FPGA and three NI USRP nodes. Fig. 19 shows the logical connections between the NI FlexRio, the NI USRP nodes and the controller (NI PXI HOST), where the upper blue section

TABLE VI: Parameters of the hardware demonstrator

\begin{tabular}{|l|c|}
\hline Parameter & Value \\
\hline Transmitter IQ channels & 6 \\
Sampling frequency & $1 \mathrm{MHz}$ \\
Oversampling factor & 4 \\
Transmitter TX freq. & $1.21 \mathrm{GHz}$ \\
Channel Emulator RX freq. & $1.21 \mathrm{GHz}$ \\
Channel Emulator TX freq. & $960 \mathrm{MHz}$ \\
User Terminal RX freq. & $960 \mathrm{MHz}$ \\
Filter roll-off factor & $0.2,0.15,0.1,0.05$ \\
Forward Error Correction & yes \\
LDPC code rate & $1 / 2,2 / 3,3 / 4,5 / 6$ \\
\hline
\end{tabular}


TABLE VII: HDL Core Resource Occupation on Kintex-7 (xc7k410TFFG-2)

\begin{tabular}{|c||c|c|c|c|}
\hline$K=N_{t}$ & DSP48E & Slices & look-up tables & Effective baud rate \\
\hline 2 & 16 & 479 & 216 & $166 \mathrm{MBd}$ \\
6 & 72 & 2019 & 2488 & $498 \mathrm{MBd}$ \\
12 & 288 & 9891 & 9938 & $996 \mathrm{MBd}$ \\
16 & 512 & 11683 & 19010 & $1.33 \mathrm{GBd}$ \\
20 & 800 & 21187 & 27602 & $1.66 \mathrm{GBd}$ \\
\hline Available & 1540 & 508400 & 254200 & \\
\hline
\end{tabular}

represents the processes implemented in the host computer and the lower yellow section represents the blocks implemented in the FPGA for fast processing. The transmitter transmits symbols modulated according to the DVB-S2X standard [305], [306], and the streams are jointly precoded by the PRECODE FPGA block. The precoder block multiplies 6 symbols from a single time slot with the precoding matrix $\mathbf{W}$ and sends the streams to the NI USRP nodes. Subsequently, each node oversamples the streams and transmits them through digital up converted (DUC) to the RF domain at a desired carrier frequency.

Fig. 20 shows an architectural block diagram of the UE implementation. A single USRP RIO FPGA unit is capable to simultaneously receive and process two information signals. The processing includes frequency acquisition, matched filtering, time synchronization, frame synchronization, fine phase tracking and CSI estimation. All the frame fields and the CSI information are passed to the host computer for further processing, and the host computer reports the CSI information to the transmitter using a custom feedback channel.

2) FPGA Accelerated Closed-Form SLP: A complete FPGA accelerated design has been developed in [83] to efficiently solve the SLP problem $\mathcal{P}_{\text {CI-VP }}$ in (30). The design is built using Vivado High-Level Synthesis (HLS) to program a closed-form algorithm into HDL core and integrate the design into an FPGA. The estimations for the resource utilization and symbol throughput of the FPGA core are demonstrated in Table VII.

The FPGA core hits the target of the symbol rate of 83

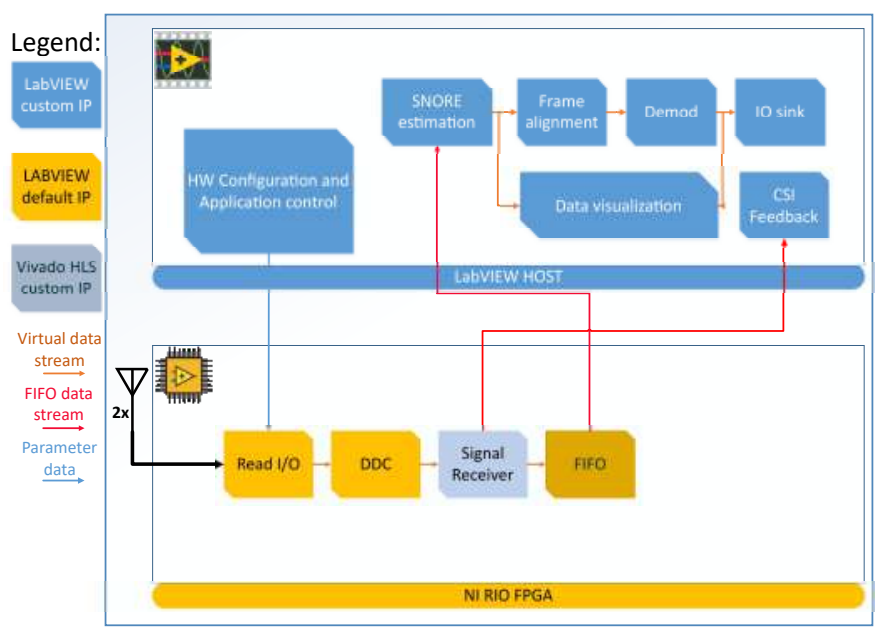

Fig. 20: Block diagram of the UE, where two input RF chains are present in a single USRP

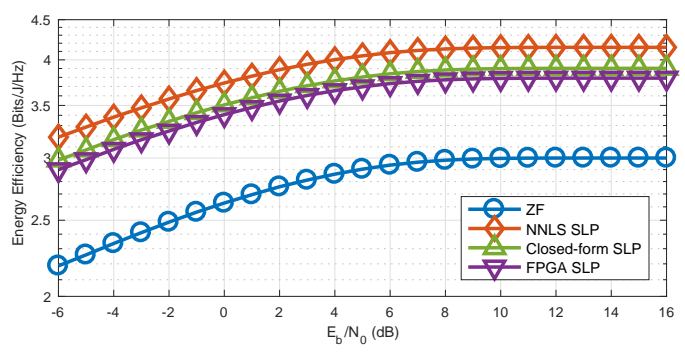

Fig. 21: Energy efficiency of ZF, FPGA SLP, CVX SLP, and closed-form SLP, 8-PSK

MSymbols per second per each beam. The motivation behind the target is the new symbols rates, which are considered in the DVB-S2X standard [305]. The estimated resource consumption by the core design are calculated for various numbers of transmitting antennas and UEs, where $K=N_{t}=$ $2,6,12,16$ and 20. For all the scenarios, the core is optimized to operate at a $166 \mathrm{MHz}$ clock $(\approx 6 \mathrm{~ns}$ per cycle $)$ with a cycle interval 2 . The clock allows to operate at the $166 \mathrm{MHz} / 2=83 \mathrm{MSymbols}$ per second symbol rate per beam. For the case of a $20 \times 20$ MU-MISO case, the design utilizes around 50 percent of the DSP blocks available at the given FPGA model (xc7k410TFFG-2) and the effective baud rate of the core reaches $1.66 \mathrm{GSymbols}$ per second.

In Fig. 21, the energy efficiency is benchmarked as a function of the energy per bit to noise power spectral density ratio $\left(E_{\mathrm{b}} / N_{0}=10 \log _{10}\left(\frac{1}{3 \sigma^{2}}\right)\right)$ for the ZF, Fast NNLS SLP and the closed-form SLP algorithm in [83] running in MATLAB and on FPGA core. The benchmark is performed on 8-PSK modulation symbols and averaged over 50 iterations of $6 \times 6$ channel matrix with a condition number, defined as $\kappa_{2}(\mathbf{H})=\|\mathbf{H}\|_{2}\left\|\mathbf{H}^{-1}\right\|_{2}$, fixed to 18. The difference between the performance of the Fast NNLS and the closedform algorithm running on MATLAB is around $2.5 \mathrm{~dB}$ due to the approximation method used in the closed-form solution. The additional $1 \mathrm{~dB}$ difference can also be observed between the MATLAB and FPGA implementations of the closed-form algorithm, which is due to the losses in fixed-point arithmetic, which are calculated on the hardware.

3) Experimental Validation: The experimental measurements of the output power are conducted using the testbed, as shown in Fig. 22a. It is evident that ZF technique generates signals with a higher averaged total transmit power than the SLP technique. The reduction in the transmit power by SLP becomes more significant as the matrix condition number increases compared with ZF.

In Fig. 22b, we can observe that the received power for $\mathrm{ZF}$ precoding is not constant for a given channel condition number. These variations come from the imperfections in the actual hardware implementation. Some of these imperfections are the limited accuracy in the CSI estimation, and its quantization error. Nevertheless, since these imperfections have the same impact on the ZF and the SLP, we can observe that the SLP approach still has gains in the received power. These gains become more frequent as the matrix condition number is increased. There are particular channel realizations in which 


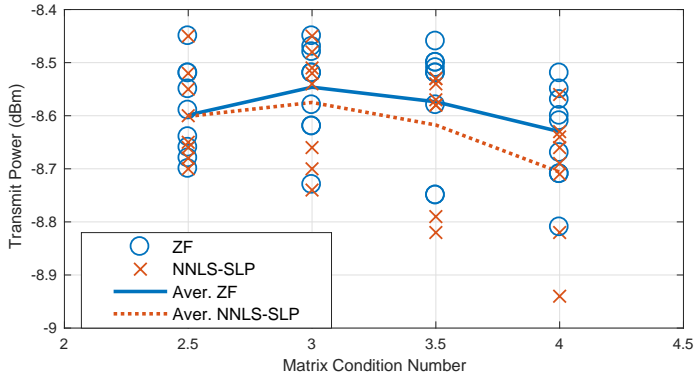

(a) Transmit powers on the output antenna ports

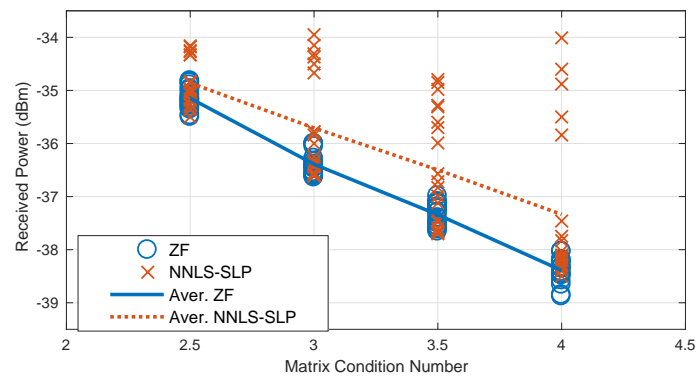

(b) Received powers on the input antenna ports

Fig. 22: Transmit/Received powers in the testbed [307]

the SLP performs the same as ZF for both receivers, and others realizations in which the perturbation vector for the optimal symbols is not strong enough.

\section{OPEn PRoblems ANd Challenges}

In this section, we discuss some remaining open problems and challenges that are to be addressed to motivate further research efforts in the field of CI exploitation and SLP techniques.

\section{A. Communication Theoretical Aspects}

While CI-based precoding techniques have been extensively studied based on optimization, the fundamental extent of the performance improvements from exploiting the CI effects is unknown, since there still lack information-theoretic studies on this topic to provide performance benchmarks. As discussed in this paper, since the precoding matrix for CI-based techniques is dependent on not only the channel knowledge but also the knowledge of the data symbols, it is therefore a modulationdependent precoding scheme, and the Shannon channel capacity expression that was derived based on the assumption of Gaussian inputs becomes no longer valid for CI-based techniques. Instead, the capacity needs to be analyzed based on the complex finite-constellation approaches [308]-[310], which are generally more complicated. Therefore, one open problem is the development of such an analytical framework that is able to offer a benchmark performance, towards which optimization-based CI techniques are designed in the future.

\section{B. Computational Complexity}

While the optimal closed-form precoding structure for $\mathrm{CI}$ precoding has been derived in [58] for PSK signaling and [60] for QAM signaling, where a low-complexity iterative approach that returns the optimal CI precoding matrix is introduced, the resulting computational complexity for CI precoding may still be too high for real-time implementation in current wireless communication systems, since CI-based SLP techniques still need to update the precoding matrix on a symbol level. This is one the most critical issues for SLP techniques that need to be addressed for their practical implementation. While there already exist SLP techniques that enjoy exactly closedform solutions in the literature [57], [60], their performances are sub-optimal. One possible solution to circumvent this drawback is to consider the relationship between the optimal precoding matrices for different input data symbol combinations. If the precoding matrix for one data symbol combination can be conveniently obtained from the precoding matrix for another data symbol combination, the computational costs for SLP techniques can be greatly reduced. However, it is still unknown whether such relationships can be obtained with low complexity or even in a closed form, which remain an open problem.

\section{Adaptive Modulation}

Another open problem is how CI approaches can be extended to adaptive modulation scenarios. In the current literature, it has been extensively discussed how current CIbased schemes can be tailored for different modulations, and particularly how various techniques can enhance the CI effect based on the specific modulation format of the data. Nonetheless, in all of the CI-based techniques currently available in the literature, only single-modulation transmission is assumed among the users, and how the CI techniques can be modified for adaptive modulation scenarios are not discussed. To motivate the use of CI techniques in practical wireless communication systems, the extension to adaptive modulation is particularly important, since typical communication systems in practice do not use a fixed modulation format, but rely on adaptive modulation schemes which adaptively adjust the modulation based on the estimated SINR (SINR estimation is further discussed below). This is eventually connected to the scheduling aspects of wireless communication systems as well. However, the current CI condition is not mathematically universal, where the CI condition for PSK modulations relies on 'phase rotation' while the CI condition for QAM modulations is based on 'symbol scaling'. It is still not known whether both of the above CI conditions can be jointly considered in adaptive modulation scenarios, and it is also not trivial whether the above two CI conditions are equivalent, both of which have not been mathematically proven. Therefore, an open challenge is to account for adaptive modulation schemes in the formulation of CI problems, which would enhance their flexibility and pave the way to practical applications.

\section{SINR Estimation}

It has been mentioned above how typical communication systems use adaptive modulation schemes based on the estimated SINR. Thus, it is particularly important to use a reliable SINR estimation scheme at the receivers [311], in 
order to feed the information back to the transmitter and choose the appropriate modulation type for the quality of wireless transmission. However, the SINR estimation is an additional challenge when CI schemes are employed. In fact, as mentioned earlier, CI-based designs lead to distorted received constellations, with some/all of the outer constellation points pushed further away from their respective detection regions. As a consequence, for multi-level modulations there will be an imbalance between the instantaneous SINR of the outer constellation points (which can be very high due to $\mathrm{CI}$ ) and that of the inner constellation points, while for single-level modulations there will also be an imbalance of the instantaneous SINR among the constellation points, since the CI effect can be different for different constellation points. Such imbalances are not taken into account by current SINR estimation schemes, which only evaluates an average SINR. Therefore, an open challenge in the context of CI precoding is to propose novel SINR estimation methods that are able to take into account the imbalance across different constellation points.

\section{E. Channel-Coded CI}

Another remaining open research area is related to the design of $\mathrm{CI}$ approaches in the presence of channel coding, or forward error correction (FEC) schemes. Since current CIbased precoding schemes are designed for uncoded wireless communication systems, their optimality is therefore only guaranteed for uncoded systems. While the use of FEC over symbol-level precoded waveforms has been assessed in some works (e.g. [219]), it is still unknown whether such a direct combination leads to the optimal coded performance. Moreover, since CI-based techniques aim to push the modulated symbols further away from the decision boundaries in order to gain benefits from CI effects, which leads to an imbalance between the constellation points for different users, it is expected that further performance gains can be achieved if an adaptive coding scheme is adopted. For users that experience a low CI effect, a robust channel coding scheme with a low code rate should be adopted, and a high code rate can be used if the users experience a high CI effect. It is therefore promising to consider a new joint design on adaptive modulation and coding specifically tailored for CI-based approaches. In addition, since $\mathrm{CI}$ precoding is aimed at distorting the received constellation in order to gain benefits from CI effects, CI precoding will not only affect the performance of FEC, as extensively discussed, but also affect the distribution of the received signals, which then requires a redesign of the decoder. Therefore, another open problem is to optimize the transmission by jointly accounting for the design of CI precoding, modulation and FEC.

\section{F. Synchronization in DA Systems}

It is well known that DA systems require strict synchronization between the distributed nodes to achieve the promised performance [312], [313]. When traditional block-level precoders are employed for the DA systems, the synchronization should be guaranteed on a block level. Nevertheless, when SLP techniques instead of traditional block-level precoders are applied at these DA systems, which require accurate synchronization on a symbol level, we conjecture that the timing misalignment will have a much more pronounced impact on symbol-level compared with block-level approaches. Therefore, it is still unknown how current synchronization approaches should be adapted to emerging SLP techniques, which remains another open problem.

\section{G. CI-based Non-Orthogonal Multiple Access (NOMA)}

Recently, non-orthogonal multiple access (NOMA) has received extensive research attention [314], [315]. Compared with orthogonal multiple access (OMA) schemes, NOMA is able to provide a significant spectral efficiency gain by multiplexing different users with different power levels. Essentially, in a multi-user NOMA network, the successive interference cancellation (SIC) approach is adopted for each user to decode the useful information, where the information for other users is regarded as noise. As can be seen, the information for other users is not exploited in traditional multi-user NOMA networks, which creates the opportunity for CI-based techniques. For example, it is not known whether additional performance gains can be achieved when NOMA is combined with CI precoding. As a step further, if the precoding matrix can be judiciously designed such that the received signals are made constructive to NOMA users, the SIC procedure might even be removed since the interference now becomes constructive in such cases. However, both of the above issues have not been well explored in the literature, which therefore still remain as open problems.

\section{H. Waveform Design}

Even though SLP has been applied in a wide range of wireless systems, user cases and architectures, its impact on waveform design is still not well understood. In principle, SLP enables the management of interference between symbols as long as we have an accurate information about the intersymbol channel. This entails that SLP can be applied for a wide range and even a combination of interference types such as ISI, ACI and multi-user interference. In some cases, the application becomes even more straightforward, since the intersymbol channel depends on the digital transceiver (e.g. filtering, sampling) and is static in contrast to dynamic propagation channels. For example, the first study in this direction looked at the combination of ISI and multi-user interference in FTN systems [219], where the artificially created ISI and multi-user interference are both exploited by CI techniques to further benefit the detection performance. In addition, CI precoding has recently been shown to be effective in transforming intercarrier interference into useful signal power when combined with SEFDM waveforms, as shown in [300]. Having witnessed the above benefits that CI-based techniques bring, the CI-based waveform design remains a promising research direction for multi-carrier transmission schemes with ACI, time-frequency packing, channels with memory, etc. 


\section{CONCLUSION}

In this paper, we have provided an extensive tutorial on interference exploitation techniques. The characterization of interference shows that interference classification into constructive or destructive is dependent on the information of both the channel and the data symbols, which implies that interference exploitation techniques need to operate on a symbol level. Moreover, the mathematical condition for achieving CI obtained via the geometry of the constellation points leads to convex formulations of PM and SB problems, which are typically more difficult to handle in the interference-reduction scenarios. The performance gains for interference exploitation in terms of transmit power savings and error rate are presented numerically, where an SNR gain of more than $10 \mathrm{~dB}$ for PSK modulations and up to $4.5 \mathrm{~dB}$ for QAM constellations is observed in terms of uncoded BER results. In terms of the transmit power savings, the gains achieved by exploiting CI can be up to $60 \%$ for QPSK, $40 \%$ for 8 PSK and $20 \%$ for 16PSK. We have also discussed the extension of CI precoding to a variety of wireless communication scenarios and included the description of a proof-of-concept testbed, where CI precoding also exhibits notable performance improvements.

\section{REFERENCES}

[1] L. Zheng and D. N. C. Tse, "Diversity and Multiplexing: A Fundamental Tradeoff in Multiple-Antenna Channels," IEEE Trans. Inf. Theory, vol. 49, no. 5, pp. 1073-1096, May 2003.

[2] M. Costa, "Writing on Dirty Paper," IEEE Trans. Inf. Theory, vol. IT-29, no. 3, pp. 439-441, May 1983.

[3] M. Joham, W. Utschick, and J. A. Nossek, "Linear Transmit Processing in MIMO Communication Systems," IEEE Trans. Sig. Process., vol. 53, no. 8 , pp. 2700-2712, Aug. 2005.

[4] T. Haustein, C. von Helmolt, E. Jorswieck, V. Jungnickel, and V. Pohl, "Performance of MIMO Systems with Channel Inversion," in IEEE 55th Vehicular Technology Conference (VTC Spring), vol. 1, Birmingham, AL, 2002.

[5] C. B. Peel, B. M. Hochwald, and A. L. Swindlehurst, "A VectorPerturbation Technique for Near-Capacity Multiantenna Multiuser Communication-part I: Channel Inversion and Regularization," IEEE Trans. Commun., vol. 53, no. 1, pp. 195-202, Jan. 2005.

[6] M. Bengtsson and B. Ottersten, "Optimal and Suboptimal Transmit Beamforming," Handbook of Antennas in Wireless Communications, Jan. 2001.

[7] M. Schubert and H. Boche, "Solution of the Multiuser Downlink Beamforming Problem with Individual SINR Constraints," IEEE Trans. Veh. Tech., vol. 53, no. 1, pp. 18-28, Jan. 2004.

[8] A. Wiesel, Y. C. Eldar, and S. Shamai (Shitz), "Linear Precoding via Conic Optimization for Fixed MIMO Receivers," IEEE Trans. Sig. Process., vol. 54, no. 1, pp. 161-176, Jan. 2006.

[9] F. Wang, X. Wang, and Y. Zhu, "Transmit Beamforming for Multiuser Downlink with Per-Antenna Power Constraints," in 2014 IEEE International Conference on Communications (ICC), Sydney, NSW, 2014.

[10] S. K. Sharma, T. E. Bogale, S. Chatzinotas, B. Ottersten, L. B. Le, and X. Wang, "Cognitive Radio Techniques under Practical Imperfections: A Survey," IEEE Commun. Surveys\&Tut., vol. 17, no. 4, pp. 18581884, fourthquarter 2015.

[11] I. Krikidis, S. Timotheou, S. Nikolaou, G. Zheng, D. W. K. Ng, and R. Schober, "Simultaneous Wireless Information and Power Transfer in Modern Communication Systems," IEEE Commun. Mag., vol. 52, no. 11, pp. 104-110, Nov. 2014.

[12] T. D. Ponnimbaduge Perera, D. N. K. Jayakody, S. K. Sharma, S. Chatzinotas, and J. Li, "Simultaneous Wireless Information and Power Transfer (SWIPT): Recent Advances and Future Challenges," IEEE Commun. Surveys\&Tut., vol. 20, no. 1, pp. 264-302, firstquarter 2018.

[13] Y. Zou, J. Zhu, X. Wang, and L. Hanzo, "A Survey on Wireless Security: Technical Challenges, Recent Advances, and Future Trends," Proceedings of the IEEE, vol. 104, no. 9, pp. 1727-1765, Sept. 2016.
[14] Y. Shiu, S. Y. Chang, H. Wu, S. C.-H. Huang, and H. Chen, "Physical layer security in wireless networks: A tutorial," IEEE Wireless Commun., vol. 18, no. 2, pp. 66-74, April 2011.

[15] A. Mukherjee, S. A. A. Fakoorian, J. Huang, and A. L. Swindlehurst, "Principles of Physical Layer Security in Multiuser Wireless Networks: A Survey," IEEE Commun. Surveys\&Tut., vol. 16, no. 3, pp. 15501573, thirdquarter 2014.

[16] N. Yang, L. Wang, G. Geraci, M. Elkashlan, J. Yuan, and M. D. Renzo, "Safeguarding 5G Wireless Communication Networks using Physical Layer Security," IEEE Commun. Mag., vol. 53, no. 4, pp. 20-27, April 2015.

[17] S. K. Sharma, T. E. Bogale, L. B. Le, S. Chatzinotas, X. Wang, and B. Ottersten, "Dynamic Spectrum Sharing in 5G Wireless Networks With Full-Duplex Technology: Recent Advances and Research Challenges," IEEE Commun. Surveys\&Tut., vol. 20, no. 1, pp. 674-707, firstquarter 2018.

[18] Z. Zhang, X. Chai, K. Long, A. V. Vasilakos, and L. Hanzo, "Full Duplex Techniques for 5G Networks: Self-Interference Cancellation, Protocol Design, and Relay Selection," IEEE Commun. Mag., vol. 53, no. 5, pp. 128-137, May 2015.

[19] D. Kim, H. Lee, and D. Hong, "A Survey of In-Band Full-Duplex Transmission: From the Perspective of PHY and MAC Layers," IEEE Commun. Surveys\&Tut., vol. 17, no. 4, pp. 2017-2046, fourthquarter 2015.

[20] F. Liu, C. Masouros, A. Li, and T. Ratnarajah, "Radar and Communication Coexistence Enabled by Interference Exploitation," in IEEE Global Communications Conference (GLOBECOM), Singapore, 2017.

[21] F. Liu, C. Masouros, A. Li, T. Ratnarajah, and J. Zhou, "MIMO Radar and Cellular Coexistence: A Power-Efficient Approach Enabled by Interference Exploitation," IEEE Trans. Sig. Process., vol. 66, no. 14, pp. 3681-3695, July 2018.

[22] L. Sun and M. Lei, "Quantized CSI-based Tomlinson-Harashima Precoding in Multiuser MIMO Systems," IEEE Trans. Wireless Commun., vol. 12, no. 3, pp. 1118-1126, Mar. 2013.

[23] C. Masouros, M. Sellathurai, and T. Ratnarajah, "Interference Optimization for Transmit Power Reduction in Tomlinson-Harashima Precoded MIMO Downlinks," IEEE Trans. Sig. Process., vol. 60, no. 5 , pp. 2470-2481, May 2012.

[24] A. Garcia-Rodriguez and C. Masouros, "Power-Efficient TomlinsonHarashima Precoding for the Downlink of Multi-User MISO Systems,' IEEE Trans. Commun., vol. 62, no. 6, pp. 1884-1896, June 2014.

[25] B. M. Hochwald, C. B. Peel, and A. L. Swindlehurst, "A VectorPerturbation Technique for Near-Capacity Multiantenna Multiuser Communication-part II: Perturbation," IEEE Trans. Commun., vol. 53, no. 3, pp. 537-544, Mar. 2005.

[26] A. Li and C. Masouros, "A Constellation Scaling Approach to Vector Perturbation for Adaptive Modulation in MU-MIMO," IEEE Wireless Commun. Lett., vol. 4, no. 3, pp. 289-292, June 2015.

[27] — - "A Two-Stage Vector Perturbation Scheme for Adaptive Modulation in Downlink MU-MIMO," IEEE Trans. Veh. Tech., vol. 65, no. 9, pp. 7785-7791, Sept. 2016.

[28] M. Alodeh, D. Spano, A. Kalantari, C. Tsinos, D. Christopoulos, S. Chatzinotas, and B. Ottersten, "Symbol-Level and Multicast Precoding for Multiuser Multiantenna Downlink: A Survey, Classification and Challenges," IEEE Commun. Surveys\&Tut., vol. 20, no. 3, pp. 1733-1757, thirdquarter 2018.

[29] J. Chen, "Alternating Minimization Algorithms for One-Bit Precoding in Massive Multiuser MIMO Systems," IEEE Trans. Veh. Tech., vol. 67 , no. 8, pp. 7394-7406, Aug. 2018

[30] M. Vu and A. J. Paulraj, "MIMO Wireless Linear Precoding," IEEE Sig. Process. Mag., vol. 24, no. 5, pp. 86-105, Sept. 2007

[31] E. Castañeda, A. Silva, A. Gameiro, and M. Kountouris, "An Overview on Resource Allocation Techniques for Multi-User MIMO Systems," IEEE Commun. Surveys\&Tut., vol. 19, no. 1, pp. 239-284, firstquarter 2017.

[32] C. Masouros, T. Ratnarajah, M. Sellathurai, C. B. Papadias, and A. K. Shukla, "Known Interference in the Cellular Downlink: A Performance Limiting Factor or a Source of Green Signal Power?" IEEE Commun. Mag., vol. 51, no. 10, pp. 162-171, Oct. 2013.

[33] G. Zheng, I. Krikidis, C. Masouros, S. Timotheou, D. A. Toumpakaris, and Z. Ding, "Rethinking the Role of Interference in Wireless Networks," IEEE Commun. Mag., vol. 52, no. 11, pp. 152-158, Nov. 2014.

[34] A. Goldsmith, Wireless Communications, 1st ed. Cambridge University Press, Aug. 2005.

[35] C. Masouros, Harvesting Signal Power from Constructive Interference in Multiuser Downlinks. Springer International Publishing, 2018, pp. $87-122$. 
[36] C. Masouros and E. Alsusa, "A Novel Transmitter-Based SelectivePrecoding Technique for DS/CDMA Systems," IEEE Sig. Process. Lett., vol. 14, no. 9, pp. 637-640, Sept. 2007.

[37] _ - "Dynamic Linear Precoding for the Exploitation of Known Interference in MIMO Broadcast Systems," IEEE Trans. Wireless Commun., vol. 8, no. 3, pp. 1396-1404, Mar. 2009.

[38] C. Masouros, "Correlation Rotation Linear Precoding for MIMO Broadcast Communications," IEEE Trans. Sig. Process., vol. 59, no. 1, pp. 252-262, Jan. 2011.

[39] C. Masouros, M. Sellathurai, and T. Ratnarajah, "Vector Perturbation based on Symbol Scaling for Limited Feedback MISO Downlinks," IEEE Trans. Sig. Process., vol. 62, no. 3, pp. 562-571, Feb. 2014.

[40] D. Kwon, W. Yeo, and D. K. Kim, "A New Precoding Scheme for Constructive Superposition of Interfering Signals in Multiuser MIMO Systems," IEEE Commun. Lett., vol. 18, no. 11, pp. 2047-2050, Nov. 2014.

[41] M. Alodeh, S. Chatzinotas, and B. Ottersten, "A Multicast Approach for Constructive Interference Precoding in MISO Downlink Channel," in IEEE International Symposium on Information Theory (ISIT), Honolulu, HI, 2014.

[42] — "Constructive Multiuser Interference in Symbol Level Precoding for the MISO Downlink Channel," IEEE Trans. Sig. Process., vol. 63, no. 9, pp. 2239-2252, May 2015.

[43] C. Masouros and G. Zheng, "Power Efficient Downlink Beamforming Optimization by Exploiting Interference," in IEEE Global Communications Conference (GLOBECOM), San Diego, CA, 2015.

[44] —_, "Exploiting Known Interference as Green Signal Power for Downlink Beamforming Optimization," IEEE Trans. Sig. Process., vol. 63, no. 14 , pp. 3628-3640, July 2015.

[45] M. Alodeh, S. Chatzinotas, and B. Ottersten, "Energy Efficient SymbolLevel Precoding in Multiuser MISO Channels," in IEEE 16th International Workshop on Signal Processing Advances in Wireless Communications (SPAWC), Stockholm, 2015.

[46] —, "Energy-Efficient Symbol-Level Precoding in Multiuser MISO based on Relaxed Detection Region," IEEE Trans. Wireless Commun., vol. 15, no. 5, pp. 3755-3767, May 2016.

[47] _ "Constructive Interference through Symbol Level Precoding for Multi-Level Modulation," in IEEE Global Communications Conference (GLOBECOM), San Diego, CA, 2015.

[48] — "Symbol-Level Multiuser MISO Precoding for Multi-Level Adaptive Modulation," IEEE Trans. Wireless Commun., vol. 16, no. 8, pp. 5511-5524, Aug. 2017.

[49] D. Spano, M. Alodeh, S. Chatzinotas, and B. Ottersten, "Per-Antenna Power Minimization in Symbol-Level Precoding," in IEEE Global Communications Conference (GLOBECOM), Washingtong, DC, 2016.

[50] Y. I. Choi, J. W. Lee, C. G. Kang, and M. Rim, "Constructive Multi-User Interference for Symbol-Level Link Adaptation: MMSE Approach," in IEEE Global Communications Conference Workshops (GLOBECOM Wkshps), Singapore, 2017.

[51] K. L. Law and C. Masouros, "Constructive Interference Exploitation for Downlink Beamforming based on Noise Robustness and Outage Probability," in IEEE International Conference on Acoustics, Speech and Signal Processing (ICASSP), Shanghai, 2016.

[52] — "Symbol Error Rate Minimization Precoding for Interference Exploitation," IEEE Trans. Commun., vol. 66, no. 11, pp. 5718-5731, Nov. 2018.

[53] D. Spano, M. Alodeh, S. Chatzinotas, and B. Ottersten, "Symbol-Level Precoding for the Nonlinear Multiuser MISO Downlink Channel," IEEE Trans. Sig. Process., vol. 66, no. 5, pp. 1331-1345, March 2018.

[54] A. Haqiqatnejad, F. Kayhan, and B. Ottersten, "Constructive Interference for Generic Constellations," IEEE Sig. Process. Lett., vol. 25, no. 4, pp. 586-590, April 2018.

[55] — - "Symbol-Level Precoding Design for Max-Min SINR in Multiuser MISO Broadcast Channels," in IEEE 19th International Workshop on Signal Processing Advances in Wireless Communications (SPAWC), Kalamata, 2018.

[56] _ - "Symbol-Level Precoding Design based on Distance Preserving Constructive Interference Regions," IEEE Trans. Sig. Process., vol. 66, no. 22 , pp. 5817-5832, Nov. 2018.

[57] — - "Power Minimizer Symbol-Level Precoding: A Closed-Form Suboptimal Solution," IEEE Sig. Process. Lett., vol. 25, no. 11, pp. 1730-1734, Nov. 2018.

[58] A. Li and C. Masouros, "Interference Exploitation Precoding Made Practical: Optimal Closed-Form Solutions for PSK Modulations," IEEE Trans. Wireless Commun., vol. 17, no. 11, pp. 7661-7676, Nov. 2018.
[59] A. Li, C. Masouros, X. Liao, Y. Li, and B. Vucetic, "Multiplexing More Data Streams in the MU-MISO Downlink by Interference Exploitation Precoding," in IEEE Wireless Communications and Networking Conference (WCNC), Seoul, 2020.

[60] A. Li, C. Masouros, Y. Li, B. Vucetic, and A. L. Swindlehurst, "Interference Exploitation Precoding for Multi-Level Modulations: Closed-Form Solutions," arXiv preprint, available online: https://arxiv.org/abs/1811.03289, 2018.

[61] Y. Liu and W. K. Ma, "Symbol-Level Precoding is Symbol-Perturbed ZF when Energy Efficiency is Sought," in IEEE International Conference on Acoustics, Speech and Signal Processing (ICASSP), Calgary, $\mathrm{AB}, 2018$.

[62] A. Li, C. Masouros, Y. Li, and B. Vucetic, "Interference Exploitation Precoding for Multi-Level Modulations," in IEEE International Conference on Acoustics, Speech and Signal Processing (ICASSP), Brighton, UK, 2019.

[63] A. Li and C. Masouros, "Exploiting Constructive Mutual Coupling in P2P MIMO by Analog-Digital Phase Alignment," IEEE Trans. Wireless Commun., vol. 16, no. 3, pp. 1948-1962, Mar. 2017.

[64] _ , "Mutual Coupling Exploitation for Point-to-point MIMO by Constructive Interference," in IEEE International Conference on Communications (ICC), Paris, 2017.

[65] O. T. Demir and T. E. Tuncer, "A New Beamformer Design Method for Multi-Group Multicasting by Enforcing Constructive Interference," in 26th European Signal Processing Conference (EUSIPCO), Rome, 2018.

[66] A. Kalantari, C. G. Tsinos, M. Soltanalian, S. Chatzinotas, W.-K. Ma, E. G. Larsson, and B. Ottersten, "M-QAM Precoder Design for MIMO Directional Modulation Transceivers," arXiv preprint, available online: https://arxiv.org/abs/1702.06878, 2018.

[67] A. Kalantari, C. Tsinos, M. Soltanalian, S. Chatzinotas, W. K. Ma, and B. Ottersten, "Spatial Peak Power Minimization for Relaxed Phase MPSK MIMO Directional Modulation Transmitter," in 25th European Signal Processing Conference (EUSIPCO), Kos, 2017.

[68] - "MIMO Directional Modulation M-QAM Precoding for Transceivers Performance Enhancement," in IEEE 18th International Workshop on Signal Processing Advances in Wireless Communications (SPAWC), Sapporo, 2017.

[69] A. Li, C. Masouros, F. Liu, and A. L. Swindlehurst, "Massive MIMO 1-Bit DAC Transmission: A Low-Complexity Symbol Scaling Approach," IEEE Trans. Wireless Commun., vol. 17, no. 11, pp. 75597575, Nov. 2018.

[70] Z. Liu, Q. Xie, K. Peng, and Z. Yang, "APSK Constellation with Gray Mapping," IEEE Commun. Lett., vol. 15, no. 12, pp. 1271-1273, Dec. 2011.

[71] M. Anedda, A. Meloni, and M. Murroni, "64-APSK Constellation and Mapping Optimization for Satellite Broadcasting Using Genetic Algorithms," IEEE Trans. Broadcasting, vol. 62, no. 1, pp. 1-9, Mar. 2016.

[72] C. Masouros, M. Sellathurai, and T. Ratnarajah, "Computationally Efficient Vector Perturbation Precoding Using Thresholded Optimization," IEEE Trans. Commun., vol. 61, no. 5, pp. 1880-1890, May 2013.

[73] — "A Low-Complexity Sequential Encoder for Threshold Vector Perturbation," IEEE Commun. Lett., vol. 17, no. 12, pp. 2225-2228, Dec. 2013.

[74] — "Maximizing Energy Efficiency in the Vector Precoded MUMISO Downlink by Selective Perturbation," IEEE Trans. Wireless Commun., vol. 13, no. 9, pp. 4974-4984, Sept. 2014.

[75] H.-S. Han, S.-H. Park, and I. Lee, "Improved Vector Perturbation with Modulo Loss Reduction for Multiuser Downlink Systems," in IEEE International Conference on Communications (ICC), Dresden, 2009.

[76] H.-S. Han, S.-H. Park, S. Lee, and I. Lee, "Modulo Loss Reduction for Vector Perturbation Systems," IEEE Trans. Commun., vol. 58, no. 12, pp. 3392-3396, Dec. 2010.

[77] Y. Hou, S. Tsukamoto, K. Yano, M. Uno, and K. Kobayashi, "Introducing Dual Regularization Parameters into Regularized Channel Inversion (RCI)-based Vector Perturbation for Modulo Loss Reduction," in 16th International Conference on Advanced Communication Technology (ICACT), Pyeongchang, 2014.

[78] C.-B. Chae, S. Shim, and R. W. Heath, "Block Diagonalized Vector Perturbation for Multiuser MIMO Systems," IEEE Trans. Wireless Commun., vol. 7, no. 11, pp. 4051-4057, Nov. 2008.

[79] R. Chen, C. Li, J. Li, and Y. Zhang, "Low Complexity User Grouping Vector Perturbation," IEEE Wireless Commun. Lett., vol. 1, no. 3, pp. 189-192, June 2012.

[80] J. Krivochiza, A. Kalantari, S. Chatzinotas, and B. Ottersten, "Low Complexity Symbol-Level Design for Linear Precoding Systems," 
in Symposium on Information Theory and Signal Processing in the Benelux, Delft University of Technology, 2017.

[81] R. Bro and S. D. Jong, "A Fast Non-Negativity-Constrained Least Squares Algorithm," Journal of Chemometrics, vol. 11, no. 5, pp. 393 401, 1997.

[82] J. Krivochiza, J. C. Merlano-Duncan, S. Andrenacci, S. Chatzinotas, and B. Ottersten, "Closed-Form Solution for Computationally Efficient Symbol-Level Precoding," in IEEE Global Communications Conference (GLOBECOM), Abu Dhabi, United Arab Emirates, 2018.

[83] — "FPGA Acceleration for Computationally Efficient SymbolLevel Precoding in Multi-User Multi-Antenna Communication Systems," IEEE Access, vol. 7, pp. 15 509-15 520, 2019.

[84] Y. Huang and D. P. Palomar, "Rank-Constrained Separable Semidefinite Programming with Applications to Optimal Beamforming," IEEE Trans. Sig. Process., vol. 58, no. 2, pp. 664-678, Feb. 2010.

[85] — - "A Dual Perspective on Separable Semidefinite Programming with Applications to Optimal Downlink Beamforming," IEEE Trans. Sig. Process., vol. 58, no. 8, pp. 4254-4271, Aug. 2010.

[86] K. L. Law, X. Wen, M. T. Vu, and M. Pesavento, "General Rank Multiuser Downlink Beamforming with Shaping Constraints Using Real-Valued OSTBC," IEEE Trans. Sig. Process., vol. 63, no. 21, pp. 5758-5771, Nov. 2015.

[87] L. Vandenberghe and S. Boyd, Convex Optimization. Cambridge University Press, 2004.

[88] W. Ryan and S. Lin, Channel Codes: Classical and Modern, 1st ed. Cambridge University Press, Oct. 2009.

[89] N. D. Sidiropoulos, T. N. Davidson, and Z.-Q. Luo, "Transmit Beamforming for Physical-Layer Multicasting," IEEE Trans. Sig. Process., vol. 54, no. 6, pp. 2239-2251, June 2006.

[90] S. X. Wu, W. K. Ma, and A. M. So, "Physical-Layer Multicasting by Stochastic Transmit Beamforming and Alamouti Space-Time Coding," IEEE Trans. Sig. Process., vol. 61, no. 17, pp. 4230-4245, Sept. 2013.

[91] S. X. Wu, Q. Li, A. M. So, and W. K. Ma, "Rank-Two Beamforming and Stochastic Beamforming for MISO Physical-Layer Multicasting with Finite-Aphabet Inputs," IEEE Sig. Process. Lett., vol. 22, no. 10 , pp. 1614-1618, Oct. 2015 .

[92] E. Karipidis, N. D. Sidiropoulos, and Z.-Q. Luo, "Quality of Service and Max-Min Fair Transmit Beamforming to Multiple Cochannel Multicast Groups," IEEE Trans. Sig. Process., vol. 56, no. 3, pp. 1268 1279, Mar. 2008

[93] D. Christopoulos, S. Chatzinotas, and B. Ottersten, "Weighted Fair Multicast Multigroup Beamforming under Per-Antenna Power Constraints," IEEE Trans. Sig. Process., vol. 62, no. 19, pp. 5132-5142, Oct. 2014.

[94] — - "Multicast Multigroup Precoding and User Scheduling for Frame-Based Satellite Communications," IEEE Trans. Wireless Commun., vol. 14, no. 9, pp. 4695-4707, Sept. 2015.

[95] Z. Xiang, M. Tao, and X. Wang, "Coordinated Multicast Beamforming in Multicell Networks," IEEE Trans. Wireless Commun., vol. 12, no. 1, pp. 12-21, Jan. 2013.

[96] Z.-Q. Luo, W. K. Ma, A. M. So, Y. Y., and S. Zhang, "Semidefinite Relaxation of Quadratic Optimization Problems," IEEE Sig. Process. Mag., vol. 27, no. 3, pp. 20-34, May 2010.

[97] P. Wolfe, "The Simplex Method for Quadratic Programming," Econometrica, vol. 27, no. 3, pp. 382-398, July 1959.

[98] G. Cornuejols and R. Tutuncu, Optimization Methods in Finance. Cambridge University Press, Dec. 2006

[99] F. Alizadeh and D. Goldfarb, "Second-Order Cone Programming," Mathematical Programming, vol. 95, no. 1, pp. 3-51, 2003.

[100] A. Ben-Israel and T. N. E. Greville, Generalized Inverses: Theory and Applications, 2nd ed. Springer International Publishing, 2003.

[101] Q. Zhang, C. He, and L. Jiang, "Per-Stream MSE based Linear Transceiver Design fo MIMO Interference Channels with CSI Error," IEEE Trans. Commun., vol. 63, no. 5, pp. 1676-1689, May 2015.

[102] J. Wang and D. P. Palomar, "Robust MMSE Precoding in MIMO Channels with Pre-Fixed Receivers," IEEE Trans. Sig. Process., vol. 58, no. 11, pp. 5802-5818, Nov. 2010.

[103] W. W. L. Ho, T. Q. S. Quek, S. Sun, and R. W. Heath, "Decentralized Precoding for Multicell MIMO Downlink," IEEE Trans. Wireless Commun., vol. 10, no. 6, pp. 1798-1809, June 2011.

[104] B. K. Chalise, S. Shahbazpanahi, A. Czylwik, and A. B. Gershman, "Robust Downlink Beamforming based on Outage Probability Specifications," IEEE Trans. Wireless Commun., vol. 6, no. 10, pp. 3498-3503, Oct. 2007.

[105] N. Vucic and H. Boche, "Robust QoS-Constrained Optimization of Downlink Multiuser MISO Systems," IEEE Trans. Sig. Process., vol. 57, no. 2, pp. 714-725, Feb. 2009.
[106] J. Wang and M. Bengtsson, "Joint Optimization of the Worst-Case Robust MMSE MIMO Transceiver," IEEE Sig. Process. Lett., vol. 18, no. 5, pp. 295-298, May 2011.

[107] G. Zheng, K.-K. Wong, and T. S. Ng, "Robust Linear MIMO in the Downlink: A Worst-Case Optimization with Ellipsoidal Uncertainty Regions," EURASIP J. Adv. Sig. Process., vol. 2008, no. 1, p. 609028 , July 2008.

[108] D. Kwon, H. S. Kang, and D. K. Kim, "Robust Interference Exploitation-based Precoding Scheme with Quantized CSIT," IEEE Commun. Lett., vol. 20, no. 4, pp. 780-783, April 2016.

[109] A. Haqiqatnejad, F. Kayhan, and B. Ottersten, "Robust Design of Power Minimizing Symbol-Level Precoder under Channel Uncertainty," arXiv preprint, available online: https://arxiv.org/abs/1805.02395, 2018.

[110] A. Salem, C. Masouros, and K.-K. Wong, "Sum Rate and Fairness Analysis for the MU-MIMO Downlink under PSK Signalling: Interference Suppression vs Exploitation," IEEE Trans. Commun., vol. 67, no. 9, pp. 6085-6098, Sept. 2019.

[111] A. Salem and C. Masouros, "Error Probability Analysis and Power Allocation for Interference Exploitation Over Rayleigh Fading Channels,' arXiv preprint, available online: https://arxiv.org/abs/1910.03102, 2019.

[112] B. Wang and K. J. R. Liu, "Advances in Cognitive Radio Networks: A Survey," IEEE J. Sel. Topics Sig. Process., vol. 5, no. 1, pp. 5-23, Feb. 2011

[113] T. Yucek and H. Arslan, "A Survey of Spectrum Sensing Algorithms for Cognitive Radio Applications," IEEE Commun. Surveys\&Tut., vol. 11, no. 1, pp. 116-130, firstquarter 2009.

[114] E. Axell, G. Leus, E. G. Larsson, and H. V. Poor, "Spectrum Sensing for Cognitive Radio: State-of-the-Art and Recent Advances," IEEE Sig. Process. Mag., vol. 29, no. 3, pp. 101-116, May 2012.

[115] R. Zhang and Y. Liang, "Exploiting Multi-Antennas for Opportunistic Spectrum Sharing in Cognitive Radio Networks," IEEE J. Sel. Topics Sig. Process., vol. 2, no. 1, pp. 88-102, Feb. 2008.

[116] Y. Richter and I. Bergel, "MMSE-SLNR Precoding for Multi-Antenna Cognitive Radio," IEEE Trans. Sig. Process., vol. 62, no. 10, pp. 2719 2729, May 2014.

[117] I. Lai, L. Zheng, C. Lee, and C. W. Tan, "Beamforming Duality and Algorithms for Weighted Sum Rate Maximization in Cognitive Radio Networks," IEEE J. Sel. Areas Commun., vol. 33, no. 5, pp. 832-847, May 2015.

[118] G. Zheng, S. Ma, K.-K. Wong, and T. Ng, "Robust beamforming in cognitive radio," IEEE Trans. Wireless Commun., vol. 9, no. 2, pp. 570-576, Feb. 2010

[119] V. Nguyen, H. V. Nguyen, and O. Shin, "An Efficient Zero-Forcing Precoding Design for Cognitive MIMO Broadcast Channels," IEEE Commun. Lett., vol. 20, no. 8, pp. 1575-1578, Aug. 2016.

[120] K. L. Law, C. Masouros, and M. Pesavento, "Transmit Precoding for Interference Exploitation in the Underlay Cognitive Radio Z-Channel," IEEE Trans. Sig. Process., vol. 65, no. 14, pp. 3617-3631, July 2017.

[121] R. Zhang, Y.-C. Liang, and S. Cui, "Dynamic Resource Allocation in Cognitive Radio Networks," IEEE Sig. Process. Mag., vol. 27, no. 3 , pp. 102-114, May 2010

[122] A. B. Gershman, N. D. Sidiropoulos, S. Shahbazpanahi, M. Bengtsson, and B. Ottersten, "Convex Optimization-based Beamforming: From Receive to Transmit and Network Designs," IEEE Sig. Process. Mag., vol. 27, no. 3, pp. 62-75, May 2010.

[123] X. Fu, J. Wang, and S. Li, "Joint Power Management and Beamforming for Base Stations in Cognitive Radio Systems," in 6th International Symposium on Wireless Communication Systems (ISWCS), Tuscany, 2009.

[124] I. Wajid, M. Pesavento, Y. C. Eldar, and D. Ciochina, "Robust Downlink Beamforming with Partial Channel State Information for Conventional and Cognitive Radio Networks," IEEE Trans. Sig. Process. vol. 61 , no. 14 , pp. 3656-3670, July 2013.

[125] E. A. Gharavol, Y. Liang, and K. Mouthaan, "Robust Downlink Beamforming in Multiuser MISO Cognitive Radio Networks with Imperfect Channel-State Information," IEEE Trans. Veh. Tech., vol. 59, no. 6, pp. 2852-2860, July 2010.

[126] S. Ma and D. Sun, "Chance Constrained Robust Beamforming in Cognitive Radio Networks," IEEE Commun. Lett., vol. 17, no. 1, pp. 67-70, Jan. 2013

[127] S. Cai and Y. Gong, "Cognitive Beamforming for Throughput Maximization with Statistical Cross Channel State Information," IEEE Commun. Lett., vol. 18, no. 11, pp. 2031-2034, Nov. 2014.

[128] Q. Li, Q. Zhang, R. Feng, L. Luo, and J. Qin, "Optimal Relay Selection and Beamforming in MIMO Cognitive Multi-Relay Networks," IEEE Commun. Lett., vol. 17, no. 6, pp. 1188-1191, June 2013. 
[129] Y. Cao and C. Tellambura, "Cognitive Beamforming in Underlay TwoWay Relay Networks with Multiantenna Terminals," IEEE Trans. Cog. Commun. Netw., vol. 1, no. 3, pp. 294-304, Sept. 2015.

[130] S. Singh, P. D. Teal, P. A. Dmochowski, and A. J. Coulson, "Robust Cognitive Radio Cooperative Beamforming," IEEE Trans. Wireless Commun., vol. 13, no. 11, pp. 6370-6381, Nov. 2014.

[131] C. Masouros and T. Ratnarajah, "Interference as a Source of Green Signal Power in Cognitive Relay Assisted Co-Existing MIMO Wireless Transmissions," IEEE Trans. Commun., vol. 60, no. 2, pp. 525-536, Feb. 2012.

[132] F. A. Khan, C. Masouros, and T. Ratnarajah, "Interference-Driven Linear Precoding in Multiuser MISO Downlink Cognitive Radio Network," IEEE Trans. Veh. Tech., vol. 61, no. 6, pp. 2531-2543, July 2012.

[133] K. L. Law, C. Masouros, and M. Pesavento, "Bivariate Probabilistic Constrained Programming for Interference Exploitation in the Cognitive Radio," in IEEE International Conference on Acoustics, Speech and Signal Processing (ICASSP), New Orleans, LA, 2017.

[134] A. Li and C. Masouros, "Hybrid Massive MIMO Unlicensed Transmission with 1-Bit Quantization," in IEEE Global Communications Conference Workshops (GLOBECOM Wkshps), Singapore, 2017.

[135] M. Alodeh, S. Chatzinotas, and B. Ottersten, "Symbol based Precoding in the Downlink of Cognitive MISO Channel," in International Conference on Cognitive Radio Oriented Wireless Networks (CROWNCOM), Doha, Qatar, 2015.

[136] A. Li and C. Masouros, "Constructive Interference Beamforming for Cooperative Dual-Hop MIMO Relay Systems," in IEEE 87th Vehicular Technology Conference (VTC Spring), Porto, Portugal, 2018.

[137] X. Zhou, R. Zhang, and C. K. Ho, "Wireless Information and Power Transfer: Architecture Design and Rate-Energy Tradeoff," IEEE Trans. Commun., vol. 61, no. 11, pp. 4754-4767, Nov. 2013.

[138] R. Zhang and C. K. Ho, "MIMO Broadcasting for Simultaneous Wireless Information and Power Transfer," IEEE Trans. Wireless Commun., vol. 12, no. 5, pp. 1989-2001, May 2013.

[139] H. Son and B. Clerckx, "Joint Beamforming Design for Multi-User Wireless Information and Power Transfer," IEEE Trans. Wireless Commun., vol. 13, no. 11, pp. 6397-6409, Aug. 2014.

[140] J. Xu, L. Liu, and R. Zhang, "Multiuser MISO Beamforming for Simultaneous Wireless Information and Power Transfer," IEEE Trans. Sig. Process., vol. 62, no. 18, pp. 4798-4810, Sept. 2014.

[141] M. R. Khandaker and K.-K. Wong, "SWIPT in MISO Multicasting Systems," IEEE Wireless Commun. Lett., vol. 3, no. 3, pp. 277-280, June 2014.

[142] Q. Shi, L. Liu, W. Xu, and R. Zhang, "Joint Transmit Beamforming and Receive Power Splitting for MISO SWIPT Systems," IEEE Trans. Wireless Commun., vol. 13, no. 6, pp. 3269-3280, June 2014.

[143] Z. Zong, H. Feng, F. R. Yu, N. Zhao, T. Yang, and B. Hu, "Optimal Transceiver Design for SWIPT in K-User MIMO Interference Channels," IEEE Trans. Wireless Commun., vol. 15, no. 1, pp. 430-445, Jan. 2016

[144] S. Lee, L. Liu, and R. Zhang, "Collaborative Wireless Energy and Information Transfer in Interference Channel," IEEE Trans. Wireless Commun., vol. 14, no. 1, pp. 545-557, Sept. 2015.

[145] J. Park and B. Clerckx, "Joint Wireless Information and Energy Transfer in a K-User MIMO Interference Channel," IEEE Trans. Wireless Commun., vol. 13, no. 10, pp. 5781-5796, Oct. 2014.

[146] J. Xiao, C. Xu, X. Huang, and J. Qin, "Robust Transceiver Design for Two-User MIMO Interference Channel with Simultaneous Wireless Information and Power Transfer," IEEE Trans. Veh. Tech., vol. 65, no. 5, pp. 3823-3828, May 2016.

[147] X. Chen, D. W. K. Ng, and H.-H. Chen, "Secrecy Wireless Information and Power Transfer: Challenges and Opportunities," IEEE Wireless Commun., vol. 23, no. 2, pp. 54-61, May 2016.

[148] Q. Shi, W. Xu, J. Wu, E. Song, and Y. Wang, "Secure Beamforming for MIMO Broadcasting with Wireless Information and Power Transfer," IEEE Trans. Wireless Commun., vol. 14, no. 5, pp. 2841-2853, Jan. 2015.

[149] L. Liu, R. Zhang, and K.-C. Chua, "Secrecy Wireless Information and Power Transfer with MISO Beamforming," IEEE Trans. Sig. Process., vol. 62, no. 7, pp. 1850-1853, April 2014.

[150] S. Wang and B. Wang, "Robust Secure Transmit Design in MIMO Channels with Simultaneous Wireless Information and Power Transfer," IEEE Sig. Process. Lett., vol. 22, no. 11, pp. 2147-2151, Nov. 2015.

[151] A. Li and C. Masouros, "Energy Efficient MIMO SWIPT by Hybrid Analog-Digital Beamforming," in IEEE 18th International Workshop on Signal Processing Advances in Wireless Communications (SPAWC), Sapporo, 2017
[152] — , "Energy-Efficient SWIPT: From Fully Digital to Hybrid AnalogDigital Beamforming," IEEE Trans. Veh. Tech., vol. 67, no. 4, pp. 33903405, April 2018.

[153] G. Zheng, C. Masouros, I. Krikidis, and S. Timotheou, "Exploring Green Interference Power for Wireless Information and Energy Transfer in the MISO Downlink," in IEEE International Conference on Communications (ICC), London, 2015.

[154] S. Timotheou, G. Zheng, C. Masouros, and I. Krikidis, "Symbol-Level Precoding in MISO Broadcast Channels for SWIPT Systems," in 23rd International Conference on Telecommunications (ICT), Thessaloniki, 2016.

[155] — _Exploiting Constructive Interference for Simultaneous Wireless Information and Power Transfer in Multiuser Downlink Systems," IEEE J. Sel. Areas Commun., vol. 34, no. 5, pp. 1772-1784, May 2016.

[156] A. Wolf and E. A. Jorswieck, "Maximization of Worse-Case Secrecy Rates in MIMO Wiretap Channels," in 44th Asilomar Conference on Signals, Systems and Computers (ACSSC), Pacific Grove, CA, 2010.

[157] Q. Li and W.-K. Ma, "Optimal and Robust Transmit Designs for MISO Channel Secrecy by Semidefinite Programming," IEEE Trans. Sig. Process., vol. 59, no. 8, pp. 3799-3812, Aug. 2011.

[158] J. Huang and A. L. Swindlehurst, "Robust Secure Transmission in MISO Channels based on Worst-Case Optimization," IEEE Trans. Sig. Process., vol. 60, no. 4, pp. 1696-1707, April 2012.

[159] S. Goel and R. Negi, "Guaranteeing Secrecy using Artificial Noise," IEEE Trans. Wireless Commun., vol. 7, no. 6, pp. 2180-2189, June 2008.

[160] W. Liao, T. Chang, W. K. Ma, and C. Chi, "QoS-based Transmit Beamforming in the Presence of Eavesdroppers: An Optimized ArtificalNoise-Aided Approach," IEEE Trans. Sig. Process., vol. 59, no. 3, pp. 1202-1216, Mar. 2011.

[161] A. Mukherjee and A. L. Swindlehurst, "Robust Beamforming for Security in MIMO Wiretap Channels with Imperfect CSI," IEEE Trans. Sig. Process., vol. 59, no. 1, pp. 351-361, Jan. 2011.

[162] Q. Li and W.-K. Ma, "Spatially Selective Artificial-Noise Aided Transmit Optimization for MISO Multi-Eves Secrecy Rate Maximization," IEEE Trans. Sig. Process., vol. 61, no. 10, pp. 2704-2717, May 2013.

[163] H. Wang, Q. Yin, and X. Xia, "Distributed Beamforming for PhysicalLayer Security of Two-Way Relay Networks," IEEE Trans. Sig. Process., vol. 60, no. 7, pp. 3532-3545, July 2012.

[164] H. Wang, M. Luo, Q. Yin, and X. Xia, "Hybrid Cooperative Beamforming and Jamming for Physical-Layer Security of Two-Way Relay Networks," IEEE Trans. Information Forensics and Security, vol. 8, no. 12, pp. 2007-2020, Dec. 2013.

[165] H. Wang and X. Xia, "Enhancing Wireless Secrecy via Cooperation Signal Design and Optimization," IEEE Commun. Mag., vol. 53, no. 12 , pp. 47-53, Dec. 2015.

[166] N. Yang, P. L. Yeoh, M. Elkashlan, R. Schober, and I. B. Collings, "Transmit Antenna Selection for Security Enhancement in MIMO Wiretap Channels," IEEE Trans. Commun., vol. 61, no. 1, pp. 144154, Jan. 2013.

[167] A. Kalantari, M. Soltanalian, S. Maleki, S. Chatzinotas, and B. Ottersten, "Directional Modulation Via Symbol-Level Precoding: A Way to Enhance Security," IEEE J. Sel. Topics Sig. Process., vol. 10, no. 8 , pp. 1478-1493, Dec. 2016.

[168] _ "Secure M-PSK Communication via Directional Modulation," in IEEE International Conference on Acoustics, Speech and Signal Processing (ICASSP), Shanghai, 2016.

[169] M. R. Khandaker, C. Masouros, and K.-K. Wong, "Constructive Interference based Secure Precoding: A New Dimension in Physical Layer Security," IEEE Trans. Information Forensics and Security, vol. 13, no. 9, pp. 2256-2268, Sept. 2018

[170] Y. Fan, X. Liao, A. Li, and V. C. M. Leung, "Secure Interference Exploitation Precoding in MISO Wiretap Channel: Destructive Region Redefinition with Efficient Solutions," arXiv preprint, available online: https://arxiv.org/abs/1909.09339, 2019.

[171] M. R. Khandaker, C. Masouros, K.-K. Wong, and S. Timotheou, "Secure SWIPT by Exploiting Constructive Interference and Artificial Noise," IEEE Trans. Commun., vol. 67, no. 2, pp. 1326-1340, Feb. 2018.

[172] D. Bharadia, E. McMilin, and S. Katti, "Full Duplex Radios," SIGCOMM Comput. Commun. Rev., vol. 43, no. 4, pp. 375-386, Aug. 2013.

[173] J. I. Choi, M. Jain, K. Srinivasan, P. Levis, and S. Katti, "Achieving Single Channel, Full Duplex Wireless Communication," in Proc. 16th Annu. Int. Conf. Mobile Comput. Netw. (MobiCom), Chicago, Illinois, 2010. 
[174] S. Huberman and T. Le-Ngoc, "Self-Interference Pricing-Based MIMO Full-Duplex Precoding," IEEE Wireless Commun. Lett., vol. 3, no. 6 , pp. 549-552, Dec. 2014.

[175] _ _ "Full-Duplex MIMO Precoding for Sum-Rate Maximization with Sequential Convex Programming," IEEE Trans. Veh. Tech., vol. 64 no. 11, pp. 5103-5112, Nov. 2015.

[176] — "MIMO Full-Duplex Precoding: A Joint Beamforming and SelfInterference Cancellation Structure," IEEE Trans. Wireless Commun., vol. 14, no. 4, pp. 2205-2217, April 2015.

[177] J. Kim, W. Choi, and H. Park, "Beamforming for Full-Duplex Multiuser MIMO Systems," IEEE Trans. Veh. Tech., vol. 66, no. 3, pp. 2423 2432, Mar. 2017.

[178] M.-W. Un, W.-K. Ma, and P. C. Ching, "Joint Transmit Beamforming Optimization and Uplink/Downlink User Selection in a Full-Duplex Multi-User MIMO System," in IEEE International Conference on Acoustics, Speech and Signal Processing (ICASSP), New Orleans, LA, 2017

[179] Y. Sun, D. W. K. Ng, and R. Schober, "Multi-Objective Optimization for Power Efficienct Full-Duplex Wireless Communication Systems," in IEEE Global Communications Conference (GLOBECOM), San Diego, CA, 2015.

[180] Y. Sun, D. W. K. Ng, J. Zhu, and R. Schober, "Multi-Objective Optimization for Robust Power Efficient and Secure Full-Duplex Wireless Communication Systems," IEEE Trans. Wireless Commun., vol. 15, no. 8, pp. 5511-5526, Aug. 2016

[181] Y. Wan, Q. Li, Q. Zhang, and J. Qin, "Optimal and Suboptimal FullDuplex Secure Beamforming Designs for MISO Two-Way Communications," IEEE Wireless Commun. Lett., vol. 4, no. 5, pp. 493-496, Oct. 2015.

[182] R. Feng, Q. Li, Q. Zhang, and J. Qin, "Robust Secure Beamforming in MISO Full-Duplex Two-Way Secure Communications," IEEE Trans. Veh. Tech., vol. 65, no. 1, pp. 408-414, Jan. 2016.

[183] F. Zhu, F. Gao, T. Zhang, K. Sun, and M. Yao, "Physical-Layer Security for Full Duplex Communications with Self-Interference Mitigation," IEEE Trans. Wireless Commun., vol. 15, no. 1, pp. 329-340, Jan. 2016.

[184] G. Zheng, "Joint Beamforming Optimization and Power Control for Full-Duplex MIMO Two-Way Relay Channel," IEEE Trans. Sig. Process., vol. 63, no. 3, pp. 555-566, Feb. 2015

[185] D. Hwang, Y. Choi, and T. Lee, "Optimization of Zero Forcing Beamformer for the Full Duplex Relay System," IEEE Commun. Lett., vol. 20, no. 8, pp. 1583-1586, Aug. 2016.

[186] X. Xu, X. Chen, M. Zhao, S. Zhou, C. Chi, and J. Wang, "PowerEfficient Distributed Beamforming for Full-Duplex MIMO Relaying Networks," IEEE Trans. Veh. Tech., vol. 66, no. 2, pp. 1087-1103, Feb. 2017.

[187] A. Almradi, P. Xiao, and K. A. Hamdi, "Hop-by-Hop ZF Beamforming for MIMO Full-Duplex Relaying with Co-Channel Interference," IEEE Trans. Commun., vol. 66, no. 12, pp. 6135-6149, Dec. 2018.

[188] M. T. Kabir, M. R. Khandaker, and C. Masouros, "Reducing SelfInterference in Full Duplex Transmission by Interference Exploitation," in IEEE Global Communications Conference (GLOBECOM), Singapore, 2017

[189] M. K. Tukur, M. R. Khandaker, and C. Masouros, "Interference Exploitation in Full Duplex Communications: Trading Interference Power for Both Uplink and Downlink Power Savings," IEEE Trans. Wireless Commun., vol. 17, no. 12, pp. 8314-8329, Dec. 2018.

[190] M. T. Kabir, M. R. Khandaker, and C. Masouros, "Robust Energy Harvesting FD Transmission: Interference Suppression versus Exploitation," IEEE Commun. Lett., vol. 22, no. 9, pp. 1866-1869, Sept. 2018.

[191] S. Zhou, M. Zhao, X. Xu, J. Wang, and Y. Yao, "Distributed Wireless Communication System: A New Architecture for Future Public Wireless Access," IEEE Commun. Mag., vol. 41, no. 3, pp. 108-113, Mar. 2003.

[192] R. W. Heath, S. Peters, Y. Wang, and J. Zhang, "A Current Perspective on Distributed Antenna Systems for the Downlink of Cellular Systems," IEEE Commun. Mag., vol. 51, no. 4, pp. 161-167, April 2013.

[193] S. Chatzinotas, M. A. Imran, and C. Tzaras, "On the Capacity of Variable Density Cellular Systems under Multicell Decoding," IEEE Commun. Lett., vol. 12, no. 7, pp. 496-498, July 2008.

[194] S. Chatzinotas, M. A. Imran, and R. Hoshyar, "Capacity limits of base station cooperation in cellular networks," Cooperative Communications for Improved Wireless Network Transmission: Frameworks for Virtual Antenna Array Applications, pp. 102-132, IGI Global 2009.

[195] W. Choi and J. G. Andrews, "Downlink Performance and Capacity of Distributed Antenna Systems in a Multicell Environment," IEEE Trans. Wireless Commun., vol. 6, no. 1, pp. 69-73, Jan. 2007.
[196] J. Zhang and J. G. Andrews, "Distributed Antenna Systems with Randomness," IEEE Trans. Wireless Commun., vol. 7, no. 9, pp. 36363646, Sept. 2008.

[197] M. M. Aldosari and K. A. Hamdi, "Performance Analysis of Energy Efficient Distributed Antenna Systems," in IEEE 81st Vehicular Technology Conference (VTC Spring), Glasgow, 2015.

[198] O. Onireti, F. Heliot, and M. A. Imran, "On the Energy EfficiencySpectral Efficiency Trade-Off of Distributed MIMO Systems," IEEE Trans. Commun., vol. 61, no. 9, pp. 3741-3753, Sept. 2013.

[199] H. Kim, S. Lee, C. Song, K. Lee, and I. Lee, "Optimal Power Allocation Scheme for Energy Efficiency Maximization in Distributed Antenna Systems," IEEE Trans. Commun., vol. 63, no. 2, pp. 431-440, Feb. 2015 .

[200] X. Chen, X. Xu, and X. Tao, "Energy Efficient Power Allocation in Generalized Distributed Antenna Systems," IEEE Commun. Lett., vol. 16, no. 7, pp. 1022-1025, July 2012 .

[201] H. Kim, E. Park, H. Park, and I. Lee, "Beamforming and Power Allocation Designs for Energy Efficiency Maximization in MISO Distributed Antenna Systems," IEEE Commun. Lett., vol. 17, no. 11, pp. 2100-2103, Nov. 2013.

[202] S.-R. Lee, S.-H. Moon, H.-B. Kong, and I. Lee, "Optimal Beamforming Schemes and Its Capacity Behavior for Downlink Distributed Antenna Systems," IEEE Trans. Wireless Commun., vol. 12, no. 6, pp. 25782587, June 2013

[203] X. Yu, J. Xu, Z. Luo, and X. Chen, "Sub-Optimal Precoding Scheme for Distributed Antenna Systems in Correlated Rayleigh Channel," Electronics Lett., vol. 49, no. 1, pp. 76-78, Jan. 2013.

[204] C. Xiao, X. Su, J. Zeng, L. Rong, and X. Xu, "Multi-Cell MMSE Precoding in Distributed Antenna System with Pilot Contamination," in IEEE/CIC International Conference on Communications in China Workshops (CIC/ICCC Wkshps), Shenzhen, 2015.

[205] J. Joung, Y. K. Chia, and S. Sun, "Energy-Efficient Large-Scale Distributed-Antenna System (L-DAS) for Multiple Users," IEEE J. Sel. Topics Sig. Process., vol. 8, no. 5, pp. 954-965, Oct. 2014.

[206] H. Q. Ngo, A. Ashikhmin, H. Yang, E. G. Larsson, and T. L. Marzetta, "Cell-Free Massive MIMO Versus Small Cells," IEEE Trans. Wireles. Commun., vol. 16, no. 3, pp. 1834-1850, Mar. 2017.

[207] T. X. Doan, H. Q. Ngo, T. Q. Duong, and K. Tourki, "On the Performance of Multigroup Multicast Cell-Free Massive MIMO," IEEE Commun. Lett., vol. 21, no. 12, pp. 2642-2645, Dec. 2017.

[208] H. Q. Ngo, L. Tran, T. Q. Duong, M. Matthaiou, and E. G. Larsson, "On the Total Energy Efficiency of Cell-Free Massive MIMO," IEEE Trans. Green Commun. Netw., vol. 2, no. 1, pp. 25-39, Mar. 2018.

[209] E. Nayebi, A. Ashikhmin, T. L. Marzetta, H. Yang, and B. D. Rao, "Precoding and Power Optimization in Cell-Free Massive MIMO Systems," IEEE Trans. Wireless Commun., vol. 16, no. 7, pp. 44454459, July 2017

[210] L. D. Nguyen, T. Q. Duong, H. Q. Ngo, and K. Tourki, "Energy Efficiency in Cell-Free Massive MIMO with Zero-Forcing Precoding Design," IEEE Commun. Lett., vol. 21, no. 8, pp. 1871-1874, Aug. 2017.

[211] R. K. Mungara, G. Caire, O. Y. Bursalioglu, C. Wang, and H. C. Papadopoulos, "Fog Massive MIMO with On-the-Fly Contamination Control," in IEEE International Symposium on Information Theory (ISIT), Vail, CO, 2018.

[212] O. Y. Bursalioglu, G. Caire, R. K. Mungara, H. C. Papadopoulos, and C. Wang, "Fog Massive MIMO: A User-Centric Seamless Hot-Spot Architecture," IEEE Trans. Wireless Commun., vol. 18, no. 1, pp. 559574, Jan. 2019.

[213] Z. Wei and C. Masouros, "Device-Centric Distributed Antenna Transmission: Secure Precoding and Antenna Selection with Interference Exploitation," IEEE Internet of Things Journal, early access, DOI:10.1109/JIOT.2019.2958420, 2020.

[214] Z. Wei, C. Masouros, K.-K. Wong, and X. Kang, "Multi-Cell Interference Exploitation: A New Dimension in Cell Coordination," arXiv preprint, available online: https://arxiv.org/abs/1901.04058, 2019.

[215] J. E. Mazo, "Faster-than-Nyquist Signaling," The Bell System Technical Journal, vol. 54, no. 8, pp. 1451-1462, Oct. 1975.

[216] A. D. Liveris and C. N. Georghiades, "Exploiting Faster-than-Nyquist Signaling," IEEE Trans. Commun., vol. 51, no. 9, pp. 1502-1511, Sept. 2003

[217] A. Modenini, F. Rusek, and G. Colavolpe, "Faster-than-Nyquist Signaling for Next Generation Communication Architectures," in 22nd European Signal Processing Conference (EUSIPCO), Lisbon, 2014.

[218] J. B. Anderson, F. Rusek, and V. Öwall, "Faster-than-Nyquist Signaling," Proceedings of the IEEE, vol. 101, no. 8, pp. 1817-1830, Aug. 2013. 
[219] D. Spano, M. Alodeh, S. Chatzinotas, and B. Ottersten, "Faster-ThanNyquist Signaling Through Spatio-Temporal Symbol-Level Precoding for the Multiuser MISO Downlink Channel," IEEE Trans. Wireless Commun., vol. 17, no. 9, pp. 5915-5928, Sept. 2018.

[220] M. Alodeh, D. Spano, S. Chatzinotas, and B. Ottersten, "Faster-thanNyquist Spatio Temporal Symbol-Level Precoding in the Downlink of Multiuser MISO Channels," in IEEE International Conference on Acoustics, Speech and Signal Processing (ICASSP), New Orleans, LA, 2017.

[221] D. Spano, S. Chatzinotas, and B. Ottersten, "Sequential SpatiaTemporal Symbol-level Precoding Enabling Faster-than-Nyquist Signaling for Multi-user MISO Systems," in 26th European Signal Processing Conference (EUSIPCO), Rome, 2018.

[222] M. Alodeh, S. Chatzinotas, and B. Ottersten, "Symbol-Level Multiuser MISO Precoding for Multi-Level Adaptive Modulation," IEEE Trans. Wireless Commun., vol. 16, no. 8, pp. 5511-5524, Aug. 2017.

[223] F. Rusek, D. Persson, B. K. Lau, E. G. Larsson, T. L. Marzetta, O. Edfors, and F. Tufvesson, "Scaling Up MIMO: Opportunities and Challenges with Very Large Arrays," IEEE Sig. Process. Mag., vol. 30, no. 1, pp. 40-60, Jan. 2013.

[224] E. G. Larsson, O. Edfors, F. Tufvesson, and T. L. Marzetta, "Massive MIMO for Next Generation Wireless Systems," IEEE Commun. Mag., vol. 52, no. 2, pp. 186-195, Feb. 2014.

[225] S. K. Mohammed and E. G. Larsson, "Single-User Beamforming in Large-Scale MISO Systems with Per-Antenna Constant-Envelope Constraints: The Doughnut Channel," IEEE Trans. Wireless Commun., vol. 11, no. 11, pp. 3992-4005, Nov. 2012.

[226] J. Pan and W. K. Ma, "Constant Envelop Precoding for SingleUser Large-Scale MISO Channels: Efficient Precoding and Optimal Designs," IEEE J. Sel. Topics Sig. Process., vol. 8, no. 5, pp. 982-995, Oct. 2014.

[227] J. Zhang, Y. Huang, J. Wang, B. Ottersten, and L. Yang, "PerAntenna Constant Envelope Precoding and Antenna Subset Selection: A Geometric Approach," IEEE Trans. Sig. Process., vol. 64, no. 23, pp. 6089-6104, Dec. 2016.

[228] S. K. Mohammed and E. G. Larsson, "Per-Antenna Constant Envelope Precoding for Large Multi-User MIMO Systems," IEEE Trans. Commun., vol. 61, no. 3, pp. 1059-1071, Mar. 2013.

[229] J. Chen, C. Wen, and K.-K. Wong, "Improved Constant Envelope Multiuser Precoding for Massive MIMO Systems," IEEE Commun. Lett., vol. 18, no. 8, pp. 1311-1314, Aug. 2014.

[230] S. K. Mohammed and E. G. Larsson, "Constant-Envelope Multi-User Precoding for Frequency-Selective Massive MIMO Systems," IEEE Wireless Commun. Lett., vol. 2, no. 5, pp. 547-550, Oct. 2013.

[231] S. Zhang, R. Zhang, and T. J. Lim, "Constant Envelope Precoding for MIMO Systems," IEEE Trans. Commun., vol. 66, no. 1, pp. 149-162, Jan. 2018.

[232] — "MISO Multicasting with Constant Envelope Precoding," IEEE Wireless Commun. Lett., vol. 5, no. 6, pp. 588-591, Dec. 2016.

[233] C. Chen and Y. Tsai, "Computationally Efficient Constant-Envelope Precoding Algorithm for MISO Multicasting Using PSK Modulation," IEEE Trans. Veh. Tech., vol. 67, no. 12, pp. 12 438-12 441, Dec. 2018.

[234] J. Chen, "Efficient Constant Envelope Precoding with Quantized Phases for Massive MU-MIMO Downlink Systems," IEEE Trans. Veh. Tech., vol. 68, no. 4, pp. 4059-4063, April 2019.

[235] P. V. Amadori and C. Masouros, "Constructive Interference based Constant Envelope Precoding," in IEEE 17th International Workshop on Signal Processing Advances in Wireless Communications (SPAWC), Edinburgh, 2016.

[236] — - "Constant Envelope Precoding by Interference Exploitation in Phase Shift Keying-Modulated Multiuser Transmission," IEEE Trans. Wireless Commun., vol. 16, no. 1, pp. 538-550, Jan. 2017.

[237] F. Liu, C. Masouros, P. V. Amadori, and H. Sun, "An Efficient Manifold Algorithm for Constructive Interference based Constant Envelope Precoding," IEEE Sig. Process. Lett., vol. 24, no. 10, pp. 1542-1546, Oct. 2017.

[238] H. Jedda, A. Mezghani, A. L. Swindlehurst, and J. A. Nossek, "Quantized Constant Envelop Precoding with PSK and QAM Signaling," IEEE Trans. Wireless Commun., vol. 17, no. 12, pp. 8022-8034, Dec. 2018.

[239] S. Domouchtsidis, C. G. Tsinos, S. Chatzinotas, and B. Ottersten, "Symbol-Level Precoding for Low Complexity Transmitter Architectures in Large-Scale Antenna Array Systems," IEEE Trans. Wireless Commun., vol. 18, no. 2, pp. 852-563, Feb. 2019.

[240] S. Sanayei and A. Nosratinia, "Antenna Selection in MIMO Systems," IEEE Commun. Mag., vol. 42, no. 10, pp. 68-73, Oct. 2004.
[241] A. F. Molisch and M. Z. Win, "MIMO Systems with Antenna Selection," IEEE Microwave Mag., vol. 5, no. 1, pp. 46-56, Mar. 2004.

[242] T. Gucluoglu and T. M. Duman, "Performance Analysis of Transmit and Receive Antenna Selection over Flat Fading Channels," IEEE Trans. Wireless Commun., vol. 7, no. 8, pp. 3056-3065, Aug. 2008.

[243] A. F. Molisch, M. Z. Win, Y.-S. Choi, and J. H. Winters, "Capacity of MIMO Systems with Antenna Selection," IEEE Trans. Wireless Commun., vol. 4, no. 4, pp. 1759-1772, July 2005.

[244] A. Gorokhov, D. A. Gore, and A. J. Paulraj, "Receive Antenna Selection for MIMO Spatial Multiplexing: Theory and Algorithms," IEEE Trans. Sig. Process., vol. 51, no. 11, pp. 2796-2807, Nov. 2003.

[245] Z. Chen, J. Yuan, and B. Vucetic, "Analysis of Transmit Antenna Selection/Maximal-Ratio Combining in Rayleigh Fading Channels," IEEE Trans. Veh. Tech., vol. 54, no. 4, pp. 1312-1321, July 2005.

[246] R. W. Heath and A. J. Paulraj, "Antenna Selection for Spatial Multiplexing Systems based on Minimum Error Rate," in IEEE International Conference on Communications (ICC), Helsinki, Finland, 2001.

[247] R. Chen, R. W. Heath, and J. G. Andrews, "Transmit Selection Diversity for Unitary Precoded Multiuser Spatial Multiplexing Systems with Linear Receivers," IEEE Trans. Sig. Process., vol. 55, no. 3, pp. 1159-1171, Mar. 2007.

[248] H. Li, L. Song, and M. Debbah, "Energy Efficiency of Large-Scale Multiple Antenna Systems with Transmit Antenna Selection," IEEE Trans. Commun., vol. 62, no. 2, pp. 638-647, Feb. 2014.

[249] X. Gao, O. Edfors, J. Liu, and F. Tufvesson, "Antenna Selection in Measured Massive MIMO Channels Using Convex Optimization," in IEEE Global Communications Conference Workshops (GLOBECOM Wkshps), Atlanta, GA, 2013.

[250] B. M. Lee, J. Choi, J. Bang, and B. Kang, "An Energy Efficient Antenna Selection for Large Scale Green MIMO Systems," in IEEE International Symposium on Circuits and Systems (ISCAS), Beijing, 2013.

[251] C. Masouros and E. Alsusa, "Transmit Antenna Selection for Partial Linear Precoding MIMO Schemes," Electronics Lett., vol. 45, no. 14, pp. 736-737, July 2009.

[252] P. V. Amadori and C. Masouros, "Power Efficient Massive MU-MIMO via Antenna Selection for Constructive Interference Optimization," in IEEE International Conference on Communications (ICC), London, 2015.

[253] _ "Interference-Driven Antenna Selection for Massive Multiuser MIMO," IEEE Trans. Veh. Tech., vol. 65, no. 8, pp. 5944-5958, Aug. 2016.

[254] — - "A Mixed-Integer Programming Approach to Interference Exploitation for Massive-MIMO," in IEEE Wireless Communications and Networking Conference Workshops (WCNC Wkshps), Barcelona, 2018.

[255] _ , "Large Scale Antenna Selection and Precoding for Interference Exploitation," IEEE Trans. Commun., vol. 65, no. 10, pp. 4529-4542, Oct. 2017.

[256] S. Domouchtsidis, C. G. Tsinos, S. Chatzinotas, and B. Ottersten, "Antenna Selection Symbol-Level Precoding for Low Complexity LargeScale Antenna Array Systems," in IEEE 23rd International Workshop on Computer Aided Modeling and Design of Communication Links and Networks (CAMAD), Barcelona, 2018.

[257] A. Alkhateeb, J. Mo, N. Gonzalez-Prelcic, and R. W. Heath, "MIMO Precoding and Combining Solutions for Millimeter-Wave Systems," IEEE Commun. Mag., vol. 52, no. 12, pp. 122-131, Dec. 2014.

[258] R. W. Heath, N. Gonzalez-Prelcic, S. Rangan, W. Roh, and A. M. Sayeed, "An Overview of Signal Processing Techniques for Millimeter Wave MIMO Systems," IEEE J. Sel. Topics Sig. Process., vol. 10, no. 3 , pp. 436-453, April 2016.

[259] R. Mendez-Rial, C. Rusu, N. Gonzalez-Prelcic, A. Alkhateeb, and R. W. Heath, "Hybrid MIMO Architectures for Millimeter Wave Communications: Phase Shifters or Switches?" IEEE Access, vol. 4 pp. 247-267, 2016.

[260] C. Pradhan, A. Li, H. Chen, Y. Li, and B. Vucetic, "Low Latency mmWave Backhaul via Traffic Dispersion," in IEEE 10th International Symposium on Turbo Codes \& Iterative Information Processing (ISTC), Hong Kong, 2018.

[261] O. E. Ayach, S. Rajagopal, S. Abu-Surra, Z. Pi, and R. W. Heath, "Spatially Sparse Precoding in Millimeter Wave MIMO Systems," IEEE Trans. Wireless Commun., vol. 13, no. 3, pp. 1499-1513, Mar. 2014.

[262] X. Yu, J. Shen, J. Zhang, and K. B. Letaief, "Alternating Minimization Algorithms for One-Bit Precoding in Massive Multiuser MIMO Systems," IEEE J. Sel. Topics Sig. Process., vol. 10, no. 3, pp. 485-500, April 2016. 
[263] A. Alkhateeb, G. Leus, and R. W. Heath, "Limited Feedback Hybrid Precoding for Multi-User Millimeter Wave Systems," IEEE Trans. Wireless Commun., vol. 14, no. 11, pp. 6481-6494, Nov. 2015.

[264] F. Sohrabi and W. Yu, "Hybrid Digital and Analog Beamforming Design for Large-Scale Antenna Arrays," IEEE J. Sel. Topics Sig. Process., vol. 10, no. 3, pp. 501-513, April 2016.

[265] X. Gao, L. Dai, S. Han, C.-L. I, and R. W. Heath, "Energy-Efficient Hybrid Analog and Digital Precoding for mmWave MIMO Systems with Large Antenna Arrays," IEEE J. Sel. Areas Commun., vol. 34, no. 4, pp. 998-1009, April 2016.

[266] L. Liang, W. Xu, and X. Dong, "Low-Complexity Hybrid Precoding in Massive Multiuser MIMO Systems," IEEE Wireless Commun. Lett., vol. 3, no. 6, pp. 653-656, Dec. 2014.

[267] A. Li and C. Masouros, "Hybrid Analog-Digital Millimeter-Wave MUMIMO Transmission with Virtual Path Selection," IEEE Commun. Lett., vol. 21, no. 2, pp. 438-441, Feb. 2017.

[268] _ "Hybrid Precoding and Combining Design for Millimeter-Wave Multi-User MIMO based on SVD," in IEEE International Conference on Communications (ICC), Paris, 2017.

[269] G. Hegde, C. Masouros, and M. Pesavento, "Analog Beamformer Design for Interference Exploitation based Hybrid Beamforming," in IEEE 10th Sensor Array and Multichannel Signal Processing Workshop (SAM Wkshps), Sheffield, 2018.

[270] — , "Interference Exploitation-based Hybrid Precoding with Robustness against Phase Errors," IEEE Trans. Wireless Commun., vol. 18, no. 7, pp. 3683-3696, July 2019.

[271] A. Li, C. Masouros, and M. Sellathurai, "Analog-Digital Beamforming for Tunable-Load MIMO by Mutual Coupling Exploitation," in IEEE 18th International Workshop on Signal Processing Advances in Wireless Communications (SPAWC), Sapporo, 2017.

[272] — , "Analog-Digital Beamforming in the MU-MISO Downlink by Use of Tunable Antenna Loads," IEEE Trans. Veh. Tech., vol. 67, no. 4, pp. 3114-3129, April 2018.

[273] A. Li, C. Masouros, and F. Liu, "Hybrid Analog-Digital Precoding for Interference Exploitation," in 26th European Signal Processing Conference (EUSIPCO), Rome, 2018.

[274] P. E. Allen and D. R. Holberg, CMOS Analog Circuit Design, 3rd ed. OUT USA, 2016.

[275] A. Mezghani, R. Ghiat, and J. A. Nossek, "Transmit Processing with Low Resolution D/A-Converters," in 16th IEEE International Conference on Electronics, Circuits and Systems (ICECS), Yasmine Hammamet, 2009.

[276] O. B. Usman, H. Jedda, A. Mezghani, and J. A. Nossek, "MMSE Precoder for Massive MIMO Using 1-Bit Quantization," in IEEE International Conference on Acoustics, Speech and Signal Processing (ICASSP), Shanghai, 2016.

[277] H. Jedda, J. A. Nossek, and A. Mezghani, "Minimum BER Precoding in 1-Bit Massive MIMO Systems," in IEEE Sensor Array and Multichannel Signal Processing Workshop (SAM), Rio de Janerio, 2016.

[278] O. Castañeda, T. Goldstein, and C. Studer, "POKEMON: A NonLinear Beamforming Algorithm for 1-Bit Massive MIMO," in IEEE International Conference on Acoustics, Speech and Signal Processing (ICASSP), New Orleans, LA, 2017.

[279] O. Castañeda, S. Jacobsson, G. Durisi, M. Coldrey, T. Goldstein, and C. Studer, "1-Bit Massive MU-MIMO Precoding in VLSI," IEEE J. Emerging Sel. Topics Circuits and Systems, vol. 7, no. 4, pp. 508-522, Dec. 2017.

[280] S. Jacobsson, G. Durisi, M. Coldrey, T. Goldstein, and C. Studer, "Quantized Precoding for Massive MU-MIMO," IEEE Trans. Commun., vol. 65, no. 11, pp. 4670-4684, Nov. 2017.

[281] L. T. N. Landau and R. C. de Lamare, "Branch-and-Bound Precoding for Multiuser MIMO Systems with 1-Bit Quantization," IEEE Wireless Commun. Lett., vol. 6, no. 6, pp. 770-773, Dec. 2017.

[282] A. L. Swindlehurst, A. K. Saxena, A. Mezghani, and I. Fijalkow, "Minimum Probability-of-Error Perturbation Precoding for the OneBit Massive MIMO Downlink," in IEEE International Conference on Acoustics, Speech and Signal Processing (ICASSP), New Orleans, LA, 2017

[283] M. Shao, Q. Li, and W. K. Ma, "One-Bit Massive MIMO Precoding via a Minimum Symbol-Error Probability Design," in IEEE International Conference on Acoustics, Speech and Signal Processing (ICASSP), Calgary, 2018.

[284] F. Sohrabi, Y. Liu, and W. Yu, "One-Bit Precoding and Constellation Range Design for Massive MIMO with QAM Signaling," IEEE J. Sel. Topics Sig. Process., vol. 12, no. 3, pp. 557-570, June 2018.

[285] H. Jedda, A. Mezghani, J. A. Nossek, and A. L. Swindlehurst, "Massive MIMO Downlink 1-Bit Precoding with Linear Programming for PSK
Signaling," in IEEE 18th International Workshop on Signal Processing Advances in Wireless Communications (SPAWC), Sapporo, 2017.

[286] A. Li, C. Masouros, and A. L. Swindlehurst, "1-Bit Massive MIMO Downlink based on Constructive Interference," in 26th European Signal Processing Conference (EUSIPCO), Rome, Italy, 2018.

[287] A. Li, F. Liu, C. Masouros, Y. Li, and B. Vucetic, "Interference Exploitation 1-Bit Massive MIMO Precoding: A Partial Branch-andBound Solution with Near-Optimal Performance," IEEE Trans. Wireless Commun., early access, DOI:10.1109/TWC.2020.2973987, 2020.

[288] C. G. Tsinos, A. Kalantari, S. Chatzinotas, and B. Ottersten, "SymbolLevel Precoding with Low Resolution DACs for Large-Scale Array MU-MIMO Systems," in IEEE 19th International Workshop on Signal Processing Advances in Wireless Communications (SPAWC), Kalamata, 2018.

[289] D. P. Bertsekas, Nonlinear Programming, 2nd ed. Athena, 1999.

[290] D. Spano, M. Alodeh, S. Chatzinotas, J. Krause, and B. Ottersten, "Spatial PAPR Reduction in Symbol-Level Precoding for the MultiBeam Satellite Downlink," in IEEE 18th International Workshop on Signal Processing Advances in Wireless Communications (SPAWC), Sapporo, 2017.

[291] D. Spano, M. Alodeh, S. Chatzinotas, and B. Ottersten, "PAPR Minimization through Spatio-Temporal Symbol-Level Precoding for the Non-Linear Multi-User MISO Channel," in IEEE International Conference on Acoustics, Speech and Signal Processing (ICASSP), Calgary, AB, 2018.

[292] A. Bereyhi, M. A. Sedaghat, S. Asaad, and R. R. Müller, "Nonlinear Precoders for Massive MIMO Systems with General Constraints," in 21th International ITG Workshop on Smart Antennas (WSA), Berlin, Germany, 2017.

[293] A. Bereyhi, M. A. Sedaghat, and R. R. Müller, "Asymptotics of Nonlinear LSE Precoders with Applications to Transmit Antenna Selection," in IEEE International Symposium on Information Theory (ISIT), Aachen, 2017.

[294] A. Bereyhi, M. A. Sedaghat, R. R. Müller, and G. Fischer, "GLSE Precoders for Massive MIMO Systems: Analysis and Applications," arXiv preprint, available online: https://arxiv.org/abs/1808.01880, 2018.

[295] A. Bereyhi, S. Asaad, R. R. Müller, and S. Chatzinotas, "RLS Precoding for Massive MIMO Systems with Nonlinear Front-End," in IEEE 20th International Workshop on Signal Processing Advances in Wireless Communications (SPAWC), Cannes, France, 2019.

[296] ETSI. Digital Video Broadcasting (DVB); Second Generation Framing Structure, Channel Coding and Modulation Systems for Broadcasting, Interactive Services, News Gathering and Other Broadband Satellite Applications; Part 1: DVB-S2.

[297] X. Yu, J. Zhang, and K. B. Letaief, "Doubling Phase Shifters for Efficient Hybrid Precoder Design in Millimeter-Wave Communication Systems," Journal of Communications and Information Networks, vol. 4, no. 2, pp. 51-67, April 2019.

[298] F. Liu, L. Zhou, C. Masouros, A. Li, W. Luo, and A. Petropulu, "Toward Dual-Functional Radar-Communication Systems: Optimal Waveform Design,” IEEE Trans. Sig. Process., vol. 66, no. 16, pp. 4264-4279, Aug. 2018.

[299] T. Xu, C. Masouros, and I. Darwazeh, "Waveform and Space Precoding for Next Generation Downlink Narrowband IoT," IEEE Internet of Things Journal, vol. 6, no. 3, pp. 5097-5107, June 2019.

[300] T. Xu, F. Liu, A. Li, C. Masouros, and I. Darwazeh, "Constructive Interference Precoding for Reliable Non-Orthogonal IoT Signaling," in IEEE Conference on Computer Communications Workshops (INFOCOM Wkshps), Paris, 2019.

[301] 3GPP TS 36.213 v.14.2.0, "LTE; Evolved Universal Terrestrial Radio Access (E-UTRA); Physical-Layer Procedure," Rel. 14, April 2017.

[302] Spirent Communications plc, "VR5 HD Spatial Channel Emulator," http://www.spirent.com/Products/VR5, April 2018.

[303] Y. S. Cho, J. Kim, W. Y. Yang, and C.-G. Kang, MIMO-OFDM Wireless Communications with MATLAB. Wiley, 2010.

[304] B. Ottersten, S. Chatzinotas, S. Andrenacci, J. C. Merlano-Duncan, an M. Alodeh, "SERENADE: Satellite Precoding Hardware Demonstrator," 2017.

[305] ETSI. Digital Video Broadcasting (DVB); Second Generation Framing Structure, Channel Coding and Modulation Systems for Broadcasting, Interactive Services, News Gathering and Other Broadband Satellite Applications; Part 2: DVB-S2 Extensions (DVB-S2X), 2015.

[306] ETSI. Digital Video Broadcasting (DVB); Implementation Guidelines for the Second Generation System for Broadcasting, Interactive Services, News Gathering and Other Broadband Satellite Applications; Part 1: DVB-S2, 2015. 
[307] J. Krivochiza, J. C. Merlano-Duncan, S. Andrenacci, S. Chatzinotas, and B. Ottersten, "Computationally and Energy Efficient Symbol-Level Precoding Communications Demonstrator," Physical Communication, vol. 28, pp. 108-115, 2018.

[308] K. Xiao, S. Guo, and M. Su, "On Capacity of MIMO Fading Channels with Finite-Size Constellation," in 5th International Conference on Wireless Communications, Networking and Mobile Computing, Beijing, 2009.

[309] T. Koch, A. Martinez, and A. G. i Fàbregas, "The Capacity Loss of Dense Constellations," in IEEE International Symposium on Information Theory (ISIT), Cambridge, MA, 2012.

[310] J. Harshan and B. S. Rajan, "On Two-User Gaussian Multiple Access Channels With Finite Input Constellations," IEEE Trans. Inf. Theory, vol. 57, no. 3, pp. 1299-1327, March 2011.

[311] S. Andrenacci, D. Spano, D. Christopoulos, S. Chatzinotas, J. Krause, and B. Ottersten, "Optimized Link Adaptation for DVB-S2X Precoded Waveforms based on SNIR Estimation," in 50th Asilomar Conference on Signals, Systems and Computers (ACSSC), Pacific Grove, CA, 2016.

[312] S. P. Stapleton and Q. Zhuang, "Time Synchronized Routing in a Distributed Antenna System," US Patent: US9439242B2, 2013.

[313] H. W. Lee, H. M. SON, H. Y. Choi, S. H. Han, and J. M. KIM, "Synchronization Method for Distributed Antenna System and Apparatus using the Same," US Patent: US9185669B2, 2012.

[314] Y. Saito, Y. Kishiyama, A. Benjebbour, T. Nakamura, A. Li, and K. Higuchi, "Non-Orthogonal Multiple Access (NOMA) for Cellular Future Radio Access," in IEEE 77th Vehicular Technology Conference (VTC Spring), Dresden, 2013.

[315] Z. Ding, Y. Liu, J. Choi, Q. Sun, M. Elkashlan, C.-L. I, and H. V. Poor, "Application of Non-Orthogonal Multiple Access in LTE and 5G Networks," IEEE Commun. Mag., vol. 55, no. 2, pp. 185-191, Feb. 2017. 